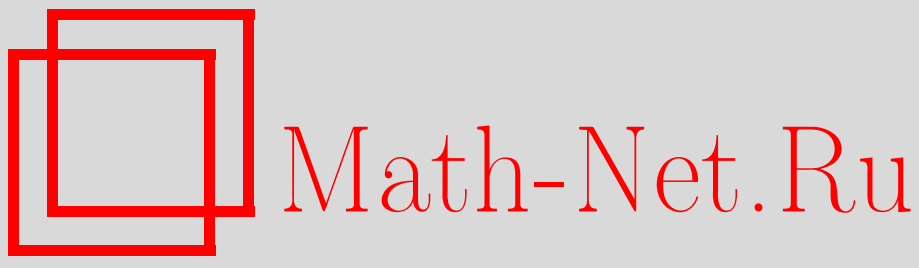

Д. И. Борисов, Асимптотики и оценки собственных элементов лапласиана с частой непериодической сменой граничных условий, Изв. РАН. Сер. матем., 2003, том 67, выпуск 6, 23-70

DOI: https://doi.org/10.4213/im459

Использование Общероссийского математического портала Math-Net.Ru подразумевает, что вы прочитали и согласны с пользовательским соглашением http://www . mathnet.ru/rus/agreement

Параметры загрузки:

IP : 3.80 .253 .173

26 апреля 2023 г., 13:43:17 
УДК 517.956

\author{
Д. И. Борисов
}

\title{
Асимптотики и оценки собственных элементов лапласиана с частой непериодической сменой граничных условий
}

\begin{abstract}
Рассматривается сингулярно возмущенная краевая задача на собственные значения оператора Лапласа в двумерной области с частой непериодической сменой граничных условий. При весьма слабых ограничениях на структуру чередования граничных условий получены первые члены асимптотических разложений собственных элементов возмущенной задачи. При еще более слабых ограничениях получены оценки скорости сходимости собственных значений возмущенной задачи.

Библиограффия: 37 наименований.
\end{abstract}

\section{Введение}

Целью настоящей работы является изучение двумерной краевой задачи с частой сменой типа граничных условий. Вначале опишем в общих чертах постановку подобных краевых задач. В области с достаточно гладкой границей рассматривается эллиптическое уравнение. На границе выделяется подмножество, состоящее из большого числа непересекаюшихся частей малой меры (см. рис. 1). На этом подмножестве задается граничное условие одного типа (например, условие Дирихле), на оставшейся части границы - другого типа (например, условие Неймана). Исследуется поведение решений такой краевой задачи, когда число частей выделенного подмножества границы неограниченно растет, а мера каждой отдельной части и расстояние между соседними частями стремится к нулю. Возможны также постановки задач, в которых описанная смена краевых условий задается не на всей гранище, а лишь на фиксированной ее части, в то время как на остальной части границы задается одно из классических краевых условий.

Вопросы усреднения эллиптических краевых задач с частой сменой граничных условий исследовались достаточно подробно (см., например, [1]-[11]). Основной целью этих работ было определение вида предельных (усредненных) задачпри минимальном наборе требований к структуре чередования граничных условий, т.е. к поведению множеств с разными краевыми условиями. В работе [1] рассматривалось уравнение Лапласа в ограниченной области с частой сменой типа граничного условия, причем было рассмотрено чередование граничных условий Дирихле и Неймана, а также случай, когда вместо граничного условия Дирихле задается краевое условие интегрального вида. Усредненные задачи в [1] были получены в предположении ряда весьма жестких ограничений на структуру чередования. В работах [2]-[9] для задач с чередованием первого краевого условия со

Работа выполнена при частичной финансовой поддержке РФФИ (гранты № 02-01-00693, № 03-01-06470), программы "Ведущие научные школы" (№ НШ-1446.2003.1) и программы "Университеты России" Минобразования РФ (УР.04.01.010).

(С) Д. И. Борисов, 2003 


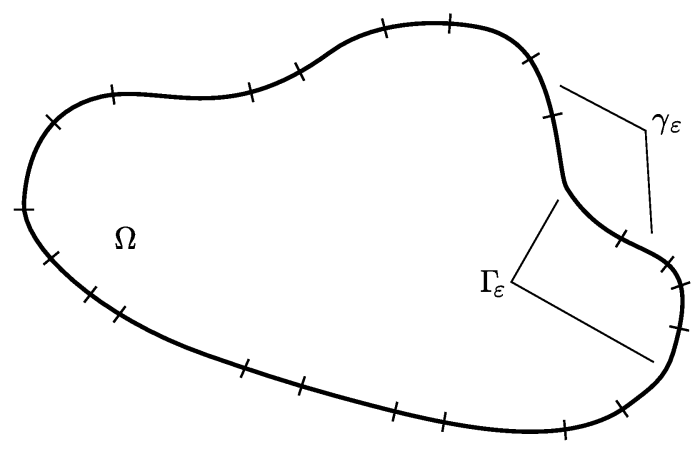

Рис. 1

вторым либо третьим краевым условием были также получены усредненные задачи и приведены достаточно простые условия, определяюшие зависимость типа усредненной задачи от структуры чередования. Случай, когда подмножество гранищы с граничным условием Дирихле имеет периодическую структуру, исследовался в [3]-[6]. Сходимость в непериодическом случае изучалась в [2], [8], [9]. Краевые задачи для нелинейных эллиптических уравнений с частой сменой типа граничного условия рассматривались в [10], [11]. В работе [10] задача решалась численно; в [11] исследовались вопросы усреднения. Результаты, полученные при изучении сходимости задач с частой сменой типа граничного условия (как периодической, так и непериодической), кратко можно сформулировать следующим образом. Краевые эллиптические задачи с частой сменой типа граничного условия при достаточно общих предположениях сходятся к задачам с классическими граничными условиями. Тип граничного условия в усредненной задаче зависит от соотношения мер частей гранищы с разными типами краевых условий в возмущенной задаче.

Усреднение краевых задач, близких к задачам со сменой типа граничных условий, изучалось в монографии [12]. Здесь рассматривались эллиптические краевые задачи во всем пространстве. Граничное условие (первое либо второе) задавалось на гранище множества, состояшего из большого количества непересекающихся малых областей, расположенных близко друг к другу. Также рассматривался случай, когда малые области заменялись на малые кривые, на которых задавалось граничное условие Неймана. Асимптотические разложения решений для задач с такой геометрией краевых условий были построены в работе [13].

Помимо определения вида усредненных задач для задач с частой сменой типа граничных условий важен и актуален также вопрос об оценках скорости сходимости. Для периодической смены краевых условий такого рода оценки были получены в [6], [15]. В случае непериодического чередования граничного условия Дирихле с третьим (либо вторым - как частный случай) краевым условием оценки скорости сходимости были выведены в [14] (предельная - задача Дирихле) и [16] (предельная - третья (вторая) краевая задача).

В последние годы появились работы, в которых строились асимптотики решений задач с периодической структурой чередования. Отметим, что факт периодичности при получении асимптотик использовался по существу. Двумерный случай исследован в работах [17]-[22]. В этих работах рассматривалось чередование граничного условия Дирихле с граничным условием Неймана. Для круга при неко- 
торых дополнительных ограничениях в [17] и [19] были построены полные степенные асимптотики собственных чисел и собственных функций оператора Лапласа в случае предельных задач Дирихле и Неймана. В работе [20] результаты [17] были обобщены, и в предположении одной лишь периодичности структуры чередования были получены полные двупараметрические асимптотические разложения собственных чисел оператора Лапласа, сходящихся к простым собственным значениям, а также были получены асимптотики соответствующих собственных функций. В работах [21] и [22] для случая произвольной области с периодической структурой чередования были построены первые члены асимптотических разложений собственных значений и собственных функций оператора Лапласа, сходящихся к простым собственным значениям второй и третьей краевых задач.

В работах [24]-[26] были рассмотрены задачи для параболического уравнения с частым чередованием первого и третьего граничных условий в предположении, что меры частей гранищы с разными граничными условиями имеют одинаковый порядок малости, что при усреднении приводило к граничному условию Дирихле. В [24], [25] для периодической, а в [26] для почти периодической смены граничных условий в различных нормах были оценены скорости сходимости и построены первые члены асимптотических разложений решений рассматриваемых задач.

В настоящей работе рассматривается краевая задача на собственные значения оператора Лапласа в произвольной двумерной области с частой, вообе говоря, непериодической сменой типа граничных условий. Изучается случай чередования граничного условия Дирихле с граничным условием Неймана. В задаче выделяются два характерных малых параметра, описывающих длины частей гранищы с первым и вторым краевыми условиями. Приведены достаточно слабые условия на множество с граничным условием Дирихле, при выполнении которых можно получить первые члены асимптотических разложений собственных значений и собственных функций возмущенной задачи в случае предельных второй или третьей краевых задач. Данные разложения асимптотичны одновременно по обоим мальм параметрам; для первых членов этих разложений получены явные формулы. В случае предельной второй краевой задачи проведено дополнительное исследование и показано, что первые члены асимптотик можно получить и при более слабых ограничениях на структуру непериодичности. Данные асимптотические разложения включают в себя как частный случай первые члены асимптотик из работ [19], [21], [22].

При дальнейшем ослаблении требований на структуру множества с граничным условием Дирихле получены двусторонние оценки отклонений собственных значений возмущенной задачи от предельных собственных значений, причем рассмотрены случаи предельных первой, второй и третьей краевых задач. Упомянутые отклонения оценены бесконечно малыми величинами, которые имеют тот же порядок малости, что и первые члены асимптотических разложений собственных значений возмущенной задачи, полученных, разумеется, при более жестких условиях на структуру чередования. Условия, наложенные на структуру чередования в [14], [16] для получения оценок скорости сходимости, более слабые, чем в настоящей работе. Вместе с тем, оценки из цитированных работ более грубые по сравнению с доказанными в настоящей статье.

Результаты предлагаемой работы были анонсированы в [23].

В заключение отметим, что вопросы усреднения и оценок скорости сходимости для трехмерных задач с частой сменой краевых условий исследовались в [1], 
[3]-[5], [7]-[11], [14]-[16]; асимптотики собственных значений оператора Лапласа в цилиндре с частой сменой граничных условий на узких полосах на боковой поверхности были построены в [27]-[29]. Отметим также, что в работах [30], [31] была рассмотрена краевая задача для уравнения Пуассона в $n$-мерном слое с частым периодическим чередованием условий Дирихле и Неймана на частях границы, стягивающихся к точке; также дополнительно предполагалось, что меры частей границы с разными типами граничных условий имеют одинаковый порядок малости. Для решения рассматриваемой задачи было получено полное асимптотическое разложение.

\section{§1. Постановка задачи и формулировка результатов}

Пусть $x=\left(x_{1}, x_{2}\right)$ - декартовы координаты, $\Omega$-произвольная ограниченная односвязная область в $\mathbb{R}^{2}$ с гладкой границей, $s$ - натуральный параметр кривой $\partial \Omega$, a $S$ - длина этой кривой, $s \in[0, S)$. Точки гранищы $\partial \Omega$ будем описывать с помошью натурального параметра, фиксировав направление обхода (против часовой стрелки) и произвольно выбрав точку на $\partial \Omega$, которой соответствует значение $s=0$. Для удобства изложения точкам границы, которым соответствуют значения $s$, близкие к $S$ или к нулю, сопоставим дополнительно значения $s-S$ и $S+s$. Пусть $N \gg 1-$ натуральное число, $\varepsilon=2 N^{-1}$ - малый положительный параметр. Для каждого значения $N$ на границе $\partial \Omega$ зададим подмножество $\gamma_{\varepsilon}$, состоящее из $N$ открытых непересекаюшихся связных частей гранищы (см. рис. 1). Опишем множество $\gamma_{\varepsilon}$ более подробно. Для каждого значения $N$ задаются точки $x_{j}^{\varepsilon} \in \partial \Omega, j=0, \ldots, N-1$, соответствуюшие значениям натурального параметра $s_{j}^{\varepsilon} \in[0, S)$, причем расстояние между любыми двумя соседними точками, измеренное вдоль границы области $\Omega$, есть величина порядка $\varepsilon$. Далее, пусть заданы два набора из $N$ функций каждый: $a_{j}(\varepsilon)$ и $b_{j}(\varepsilon), j=0, \ldots, N-1$, где функции $a_{j}$ и $b_{j}$ неотрицательны и ограничены. Множество $\gamma_{\varepsilon}$ определяется следуюшим образом:

$$
\gamma_{\varepsilon}=\bigcup_{j=0}^{N-1} \gamma_{\varepsilon, j}, \quad \gamma_{\varepsilon, j}=\left\{x:-\varepsilon a_{j}(\varepsilon)<s-s_{j}^{\varepsilon}<\varepsilon b_{j}(\varepsilon)\right\}, \quad j=0, \ldots, N-1 .
$$

Без ограничения обшности предположим, что множества $\gamma_{\varepsilon, j}$ не пересекаются.

ЗАмечАниЕ 1.1. Мы не исключаем ситуацию, когда для некоторых значений $\varepsilon$ и $j$ функция $a_{j}$ или $b_{j}$ обрашается в нуль. В этом случае точка $x_{j}^{\varepsilon}$ не принадлежит множеству $\gamma_{\varepsilon, j}$.

Рассмотрим сингулярно возмущенную краевую задачу на собственные значения:

$$
\begin{aligned}
-\Delta \psi_{\varepsilon} & =\lambda_{\varepsilon} \psi_{\varepsilon}, & & x \in \Omega, \\
\psi_{\varepsilon} & =0, & & x \in \gamma_{\varepsilon}, \\
\frac{\partial \psi_{\varepsilon}}{\partial \nu} & =0, & & x \in \Gamma_{\varepsilon},
\end{aligned}
$$

где $\nu$ - внешняя нормаль к границе $\partial \Omega, \Gamma_{\varepsilon}=\partial \Omega \backslash \bar{\gamma}_{\varepsilon}$. Целью настоящей работы является исследование поведения решений возмущенной задачи при $\varepsilon \rightarrow 0$ (или, эквивалентно, $N \rightarrow \infty)$.

Положим $a_{N}(\varepsilon) \equiv a_{0}(\varepsilon), b_{N}(\varepsilon) \equiv b_{0}(\varepsilon), s_{N}^{\varepsilon} \equiv s_{0}^{\varepsilon}$. Выражения вида $f^{\prime}$ обозначают производные по $s$.

Всюду далее будем предполагать выполнение следуюшего условия. 
(C0) Существует функция $\theta_{\varepsilon}(s), \theta_{\varepsilon}:[0, S] \rightarrow[0,2 \pi], \theta_{\varepsilon}(0)=0, \theta_{\varepsilon}(S)=2 \pi$, такая, что

$$
\theta_{\varepsilon}\left(s_{j}^{\varepsilon}\right)=\theta_{\varepsilon}\left(s_{0}^{\varepsilon}\right)+\varepsilon \pi j, \quad j=0, \ldots, N-1,
$$

$\theta_{\varepsilon}^{\prime} \in C^{\infty}(\partial \Omega), \quad 0<c_{1} \leqslant \theta_{\varepsilon}^{\prime}(s) \leqslant c_{2}$, әде $c_{1}, c_{2}$ - некоторье константы, не зависящие от $\varepsilon$ и . При $\varepsilon \rightarrow 0$ функция $\theta_{\varepsilon}(s)$ сходится в $C^{1}[0, S]$ к некоторой функиии $\theta_{0}(s), \theta_{0}^{\prime} \in C^{\infty}(\partial \Omega)$. Норма $\left\|\theta_{\varepsilon}^{\prime}\right\|_{C^{3}(\partial \Omega)}$ ограничена по $\varepsilon$.

Геометрически условие $(\mathrm{C} 0)$ означает, что гранищу $\partial \Omega$ можно гладко и взаимно однозначно отобразить на окружность единичного радиуса так, что при этом отображении множество точек $\left\{x_{j}^{\varepsilon}\right\}$ переходит в периодическое множество точек, делящее единичную окружность на $N$ дуг длиной $\varepsilon \pi$ каждая, причем данное отображение может зависеть от $\varepsilon$. На характер этой зависимости налагаются лишь условия сходимости $\theta_{\varepsilon}$ и ограниченности $\left\|\theta_{\varepsilon}^{\prime}\right\|_{C^{3}(\partial \Omega)}$.

ЗАмЕчАниЕ 1.2. Следует подчеркнуть, что непериодическая структура смены граничных условий не связана с отображением $\theta_{\varepsilon}$, т. е. отображение $\theta_{\varepsilon}$, вообще говоря, не переводит $\gamma_{\varepsilon}$ в периодическое множество. Пусть, например, $\Omega$ - единичный круг с центром в нуле, $x_{j}^{\varepsilon}=(\cos \varepsilon \pi j, \sin \varepsilon \pi j), s_{j}^{\varepsilon}=\varepsilon \pi j, a_{j}(\varepsilon)=\varepsilon j(1+\varepsilon \sin j) / 2$, $b_{j}(\varepsilon)=1-\varepsilon j / 2$. Здесь множество $\gamma_{\varepsilon}$ представляет собой объединение дуг разной длины, лежаших на гранище $\partial \Omega$, причем $j$-я дуга содержит точку $\{r=1, \theta=\varepsilon \pi j\}$, $(r, \theta)$ - полярные координаты, но не центрирована относительно этой точки. Условие $(\mathrm{C} 0)$ для такого множества выполнено при $\theta_{\varepsilon}(s) \equiv \theta_{0}(s) \equiv s \equiv \theta, a^{j}(\varepsilon)=a_{j}(\varepsilon)$, $b^{j}(\varepsilon)=b_{j}(\varepsilon)$.

Собственные значения возмущенной задачи занумеруем в порядке неубывания с учетом кратности: $\lambda_{\varepsilon}^{1} \leqslant \lambda_{\varepsilon}^{2} \leqslant \cdots \leqslant \lambda_{\varepsilon}^{k} \leqslant \cdots$. Соответствуюшие собственные функции $\psi_{\varepsilon}^{k}$ ортонормируем в $L_{2}(\Omega)$.

Первой частью основных результатов статьи являются оценки скорости сходимости, приведенные в формулировках следующих трех теорем.

Теорема 1.1. Пусть выполнено условие (C0) и

1) существует положительная ограниченная функиия $\eta=\eta(\varepsilon)$, удовлетворяющая равенству

$$
\lim _{\varepsilon \rightarrow 0} \varepsilon \ln \eta(\varepsilon)=0
$$

такая, что имеют место оценки

$$
2 c_{1}^{-1} \eta(\varepsilon) \leqslant \min _{j} a_{j}(\varepsilon)+\min _{i} b_{i}(\varepsilon),
$$

где $c_{1}$ - константа из условия (C0);

2) существует $c_{3}>0$, для которого норма Гёльдера $\left\|\theta_{\varepsilon}^{\prime}\right\|_{C^{3+c_{3}(\partial \Omega)}}$ ограничена по $\varepsilon$.

Тогда собственные значения $\lambda_{\varepsilon}^{k}$ возмущенной задачи сходятся $к$ собственным.м значениям $\lambda_{0}^{k}$ (расположсенным в порядке неубывания с учетом кратности) предельной задачи

$$
-\Delta \psi_{0}=\lambda_{0} \psi_{0}, \quad x \in \Omega, \quad \psi_{0}=0, \quad x \in \partial \Omega,
$$

и справедливь оценки

$$
C_{k, 1} \varepsilon \ln \sin \eta(\varepsilon)-C_{k, 2}|\varepsilon \ln \eta(\varepsilon)|^{3 / 2}(\pi / 2-\eta(\varepsilon)) \leqslant \lambda_{\varepsilon}^{k}-\lambda_{0}^{k} \leqslant 0,
$$


где $C_{k, i}$ - некоторые положительные константы, не зависящие от $\varepsilon, ~ a$ функиия $\eta(\varepsilon)$ ограничена сверху числом $\pi / 2$.

ТЕОРЕМА 1.2. Пусть выполнено условие (C0) и существуют положительнье функиии $\eta=\eta(\varepsilon)$ и $\eta_{0}=\eta_{0}(\varepsilon)$, удовлетворяющие равенствам

$$
\lim _{\varepsilon \rightarrow 0} \varepsilon \ln \eta_{0}(\varepsilon)=0
$$

$u$

$$
\lim _{\varepsilon \rightarrow 0}(\varepsilon \ln \eta(\varepsilon))^{-1}=-A
$$

где $A=$ const $>0$, такие, что имеют место оценки

$$
2 c_{1}^{-1} \eta_{0} \eta \leqslant a_{j}+b_{j} \leqslant 2 c_{2}^{-1} \eta, \quad j=0, \ldots, N-1
$$

с константами с $c_{1}$ и $c_{2}$ из условия $(\mathrm{C} 0)$.

Тогда собственные значения $\lambda_{\varepsilon}^{k}$ возмущенной задачи сходятся $\kappa$ собственным значениям $\lambda_{0}^{k}$ (расположсенным в порядке неубывания с учетом кратности) предельной задачи

$$
-\Delta \psi_{0}=\lambda_{0} \psi_{0}, \quad x \in \Omega, \quad\left(\frac{\partial}{\partial \nu}+A \theta_{0}^{\prime}(s)\right) \psi_{0}=0, \quad x \in \partial \Omega,
$$

и справедливы оценки

$$
\begin{aligned}
C_{k, 1} \mu(\varepsilon)+C_{k, 2} \varepsilon \ln \eta_{0}(\varepsilon)-C_{k, 3} \varepsilon-C_{k, 4} \sigma(\varepsilon) \\
\leqslant \lambda_{\varepsilon}^{k}-\lambda_{0}^{k} \leqslant C_{k, 5} \mu(\varepsilon)+C_{k, 6} \varepsilon^{3 / 2}+C_{k, 7} \sigma(\varepsilon),
\end{aligned}
$$

где $\mu=\mu(\varepsilon)=-(\varepsilon \ln \eta(\varepsilon))^{-1}-A, \quad \sigma(\varepsilon)=\left\|\theta_{\varepsilon}^{\prime}-\theta_{0}^{\prime}\right\|_{C(\partial \Omega)}, C_{k, i}-$ некоторие положительные константы, не зависящие от $\varepsilon$, а функция $\eta_{0}(\varepsilon)$ ограничена сверху единицей.

ТЕОРЕМА 1.3. Пусть выполнено условие (C0) и существует положсительная функция $\eta=\eta(\varepsilon)$, удовлетворяющая равенству (1.5) при $A=0$, такая, что имеют место оченки

$$
a_{j}+b_{j} \leqslant 2 c_{2}^{-1} \eta, \quad j=0, \ldots, N-1
$$

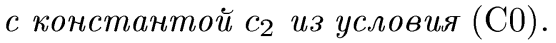

Тогда собственные значения $\lambda_{\varepsilon}^{k}$ возмущенной задачи сходятся к собственнымм значениям $\lambda_{0}^{k}$ задачи (1.6) при $A=0$ и справедливы оценки

$$
0 \leqslant \lambda_{\varepsilon}^{k}-\lambda_{0}^{k} \leqslant C_{k} \mu(\varepsilon),
$$

где $\mu=\mu(\varepsilon)=-(\varepsilon \ln \eta(\varepsilon))^{-1}, C_{k}$ - некоторые положстельнье константы, не зависящие от $\varepsilon$.

Поясним геометрический смысл условий теорем 1.1-1.3. Оценки для сумм $a_{j}+b_{j}$ в условиях данных теорем являются ограничениями на длины отдельных составляющих множества $\gamma_{\varepsilon}$ и позволяют множествам $\gamma_{\varepsilon, j}$ иметь длины paзных порядков. Более того, благодаря оценке для $a_{j}+b_{j}$ в условии теоремы 1.3 допустима ситуация, когда для некоторых $\varepsilon$ и $j$ выполнено равенство $a_{j}(\varepsilon)+b_{j}(\varepsilon)=0$, т. е. 
соответствуюшее множество $\gamma_{\varepsilon, j}$ пустое, и в окрестности точки $x_{j}^{\varepsilon}$ задается граничное условие Неймана. Следует отметить, что константы в упомянутых оценках из условий теорем 1.1-1.3 могут быть произвольными, однако их всегда можно выбрать указанным образом путем умножения функций $\eta$ и $\eta_{0}$ на подходящие числа.

Вторая часть основных результатов настоящей работы - это асимптотические разложения собственных элементов возмущенной задачи. Ясно, что требования на множество $\gamma_{\varepsilon}$, необходимые для возможности построения данных асимптотик, должны быть более жесткие по сравнению с условиями теорем 1.1-1.3. Одно из таких требований на множество $\gamma_{\varepsilon}$, налагаемое дополнительно к условию (С0), выглядит следующим образом.

(C1) Существует полохительная ограниченная функиия $\eta=\eta(\varepsilon)$ такая, что выполнень оценки

$$
c_{4} \eta(\varepsilon) \leqslant a_{j}(\varepsilon)+b_{j}(\varepsilon) \leqslant 2 c_{2}^{-1} \eta(\varepsilon), \quad j=0, \ldots, N-1,
$$

әде положительная константа $c_{4}$ не зависит от $\varepsilon, \eta u j$.

Геометрически условие $(\mathrm{C} 1)$ означает, что при $\varepsilon \rightarrow 0$ все множества $\gamma_{\varepsilon, j}$ имеют длины одинакового порядка $\varepsilon \eta$, что, однако, вовсе не означает точного равенства этих длин. Отметим, что в правой части неравенства из этого условия можно было бы написать некоторую константу $c_{5}$, которой, однако, легко придать указанное значение, умножая $\eta(\varepsilon)$ на соответствующее число.

Для того чтобы сформулировать основные результаты работы об асимптотических разложениях, понадобятся некоторые вспомогательные факты и дополнительные обозначения.

Функцию $\theta_{\varepsilon}$ продолжим на значения $s \in[-S, 2 S)$ по правилу $\theta_{\varepsilon}(s)=\theta_{\varepsilon}(s-$ $k S)+2 \pi k, s \in[k S,(k+1) S), k=-1,0,1$. Обозначим:

$$
\begin{array}{ll}
d_{j}(\varepsilon)=\frac{a_{j}(\varepsilon)+b_{j}(\varepsilon)}{2 \eta(\varepsilon)}, & d^{j}(\varepsilon)=\frac{\theta_{\varepsilon}\left(s_{j}^{\varepsilon}+\varepsilon b_{j}(\varepsilon)\right)-\theta_{\varepsilon}\left(s_{j}^{\varepsilon}-\varepsilon a_{j}(\varepsilon)\right)}{2 \varepsilon \eta(\varepsilon)}, \\
\delta_{j}(\varepsilon)=d_{j+1}(\varepsilon)-d_{j}(\varepsilon), & \delta^{j}(\varepsilon)=d^{j+1}(\varepsilon)-d^{j}(\varepsilon) .
\end{array}
$$

Пусть $\chi(t)$ - бесконечно дифференцируемая срезающая функция, равная единице при $t<1 / 4$ и нулю при $t>3 / 4$ и принимаюшая значения из отрезка $[0,1]$. Введем еще одну функцию $g_{\varepsilon}(\theta)$ следующим образом:

$$
g_{\varepsilon}(\theta)=d^{j+1}(\varepsilon)-\chi\left(\left(\theta-\theta_{\varepsilon}\left(s_{j}^{\varepsilon}\right)\right) /(\varepsilon \pi)\right) \delta^{j}(\varepsilon)
$$

при $\varepsilon \pi j \leqslant \theta-\theta_{\varepsilon}\left(s_{\varepsilon}^{0}\right) \leqslant \varepsilon \pi(j+1), j=0, \ldots, N-1$. Собственные значения задачи (1.6), как и ранее, занумеруем в порядке неубывания с учетом кратности: $\lambda_{0}^{1} \leqslant$ $\lambda_{0}^{2} \leqslant \cdots \leqslant \lambda_{0}^{k} \leqslant \cdots$, а соответствующие собственные функции $\psi_{0}^{k}$ ортонормируем в $L_{2}(\Omega)$.

Следующее утверждение носит вспомогательный характер и будет доказано в $\oint 2$. 
ЛЕмма 1.1. Пусть $\left\|\theta_{\varepsilon}^{\prime}-\theta_{0}^{\prime}\right\|_{C(\partial \Omega)} \rightarrow 0$. Тогда собственные значения $\Lambda_{0}^{k}=$ $\Lambda_{0}^{k}(\mu, \varepsilon)$ задачи

$$
\begin{gathered}
-\Delta \Psi_{0}^{k}=\Lambda_{0}^{k} \Psi_{0}^{k}, \quad x \in \Omega, \\
\left(\frac{\partial}{\partial \nu}+(A+\mu) \theta_{\varepsilon}^{\prime}(s)\right) \Psi_{0}^{k}=0, \quad x \in \partial \Omega,
\end{gathered}
$$

где $A \geqslant 0$, расположсенные в порядке неубывания с учетом кратности, сходятся $\kappa$ собственным значениям $\lambda_{0}^{k}$ задачи (1.6) при $(\varepsilon, \mu) \rightarrow 0$. Для каждого фиксированного значения $\varepsilon$ собственные значения $\Lambda_{0}^{k} u$ соответствующие ортонормированные в $L_{2}(\Omega)$ собственные функиии $\Psi_{0}^{k}$ голоморфны по $\mu$ (последние - в норме $\left.H^{1}(\Omega)\right)$. Если $\Lambda_{0}^{k}$ - кратное собственное значение, то соответствующие ему собственнье функции дополнительно можно выбрать ортогональными в скалярном произведении $L_{2}(\partial \Omega)$ с весом $\theta_{\varepsilon}^{\prime}$.

Сформулируем теперь вторую часть основных результатов работы.

ТеОРема 1.4. Пусть выполнены условия (C0), (C1), а также равенства (1.5) при $A \geqslant 0$ для функиии $\eta$ из (C1) и

$$
\max _{j}\left|\delta_{j}(\varepsilon)\right|=o\left(\varepsilon^{1 / 2}(A+\mu)^{-1}\right),
$$

где $\mu=\mu(\varepsilon)=-(\varepsilon \ln \eta(\varepsilon))^{-1}-A$. Тогда для каждого $k$ собственное значение $\lambda_{\varepsilon}^{k}$ возмущенной задачи сходится $\kappa$ собственному значению $\lambda_{0}^{k}$ предельной задачи (1.6) и имеет асимптотику:

$$
\begin{gathered}
\lambda_{\varepsilon}^{k}=\Lambda_{0}^{k}(\mu, \varepsilon)+\varepsilon \Lambda_{1}^{k}(\mu, \varepsilon)+o(\varepsilon(A+\mu)), \\
\Lambda_{1}^{k}(\mu, \varepsilon)=(A+\mu)^{2} \int_{\partial \Omega}\left(\Psi_{0}^{k}(x, \mu, \varepsilon)\right)^{2} \ln g_{\varepsilon}\left(\theta_{\varepsilon}(s)\right) \theta_{\varepsilon}^{\prime}(s) d s,
\end{gathered}
$$

где $\Lambda_{0}^{k}$ и $\Psi_{0}^{k}$ удовлетворяют утверждению леммы 1.1. Функция $\Lambda_{1}^{k}$ неположительна и голоморфна по $\mu$ для каждого фиксированного значения $\varepsilon$.

ЗАМЕчАниЕ 1.3 . В случае простого предельного собственного значения $\lambda_{0}^{k}$ в следуюшем параграфе будет дополнительно доказано, что коэффициенты рядов Тейлора функций $\Lambda_{0}^{k}, \Psi_{0}^{k}$ по степеням $\mu$ непрерывны при $\varepsilon \rightarrow 0$, а сами функции мажорируются голоморфными по $\mu$ функциями, не зависящими от $\varepsilon$. Функция $\Psi_{0}^{k}$ мажорируется в смысле нормы $H^{1}(\Omega)$. Также будет показано, что $\Psi_{0}^{k}$ сходится к $\psi_{0}^{k}$ в $H^{1}(\Omega)$ при $(\varepsilon, \mu) \rightarrow(0,0)$.

ЗАмечаниЕ 1.4 . Величины $\delta_{j}$ характеризуют разность длин двух соседних множеств $\gamma_{\varepsilon, j+1}$ и $\gamma_{\varepsilon, j}$, поэтому равенство (1.9) фактически означает, что длины двух соседних составляющих $\gamma_{\varepsilon}$ не очень сильно отличаются друг от друга.

Наряду с асимптотиками $\lambda_{\varepsilon}^{k}$ в работе доказаны утверждения об асимптотиках соответствуюших собственных функций $\psi_{\varepsilon}^{k}$ при условиях теоремы 1.4. Для формулировки этих утверждений требуется ввести ряд дополнительных обозначений, и поэтому удобнее сформулировать их в конце $§ 2$ (см. теоремы $2.1,2.2$ ).

В следующей теореме приведены асимптотики собственных значений возмущенной задачи в случае нарушения равенства (1.9) и сохранения остальных условий теоремы 1.4. 
ТЕОРема 1.5. Пусть выполнены условия (C0), (C1) и равенство (1.5) при $A \geqslant 0$ для функиии $\eta$ из (C1). Тогда собственное значение $\lambda_{\varepsilon}^{k}$ возмущенной задачи сходится $к$ собственному значению $\lambda_{0}^{k}$ предельной задачи (1.6) и имеет асимптотику:

$$
\lambda_{\varepsilon}^{k}=\lambda_{0}^{k}+\mu \int_{\partial \Omega}\left(\psi_{0}^{k}(x)\right)^{2} \theta_{0}^{\prime}(s) d s+O\left(\mu^{2}+\mu\left(\sigma+\varepsilon^{3 / 2}\right)+A\left(\sigma+\varepsilon^{1 / 2}\right)\right),
$$

где в случае кратного собственного значения $\lambda_{0}^{k}$ соответствующие собственные функции дополнительно выбраны ортогональными в $L_{2}(\partial \Omega)$ с ве$\operatorname{coм} \theta_{0}^{\prime}, \sigma=\sigma(\varepsilon)=\left\|\theta_{\varepsilon}^{\prime}-\theta_{0}^{\prime}\right\|_{C(\partial \Omega)}$.

Асимптотика (1.12) конструктивна при $A=0$, а в случае $A>0$ - при $\sigma+$ $\varepsilon^{1 / 2}=o(\mu)$.

Утверждение об асимптотиках собственных функций $\psi_{\varepsilon}^{k}$ в условиях последней теоремы будет доказано в $\S 3$ (см. теорему 3.1 ).

Настоящая работа имеет следующую структуру. В $\S 2$ доказаны теорема 1.4 и в ее условиях - теоремы 2.1, 2.2 об асимптотиках собственных функций возмушенной задачи. В $\S 3$ доказаны теорема 1.5 , а также теорема 3.1 об асимптотиках собственных функций возмущенной задачи в условиях теоремы 1.5. В $\S 4$ установлена справедливость вспомогательного утверждения об асимптотиках собственных значений в случае предельной задачи Дирихле. Это вспомогательное утверждение будет использовано в $\S 5$ при доказательстве теоремы 1.1. Кроме того, в $\S 5$ также будут доказаны теоремы $1.2,1.3$.

\section{$\S 2$. Асимптотики собственных элементов возмущенной задачи в условиях теоремы 1.4}

В настоящем параграфе мы получим асимптотики собственных элементов возмущенной задачи. Прежде установим справедливость ряда вспомогательных утверждений.

ДокАЗАТЕльСтво лЕммы 1.1. Краевая задача (1.7), (1.8) является регулярно возмущенной. Сходимость собственных значений и утверждаемая голоморфность собственных элементов по $\mu$ легко устанавливаются, если свести задачу (1.7), $(1.8)$ к операторному уравнению в $H^{1}(\Omega)$ и применить результаты работы [35]. Cохраняя все указанные свойства, собственные функции $\Psi_{0}^{k}$ можно выбрать ортонормированными в $L_{2}(\Omega)$. Согласно теореме о диагонализации двух квадратичных форм собственные функции, соответствуюшие кратному собственному значению, можно дополнительно выбрать ортогональными в $L_{2}(\partial \Omega)$ с весом $\theta_{\varepsilon}^{\prime}$. Поскольку $\theta_{\varepsilon}^{\prime}$ не зависит от $\mu$, то такая дополнительная ортогонализация сохранит голоморфность по $\mu$ данных собственных функций. Лемма доказана.

Если $\lambda_{0}^{k}$-простое собственное значение задачи (1.6), то к нему сходится только одно собственное значение $\Lambda_{0}^{k}$ задачи $(1.7),(1.8)$, а соответствующая собственная функция $\Psi_{0}^{k}$ сходится к $\psi_{0}^{k}$ в $H^{1}(\Omega)$. Кроме того, данные собственные элементы $\Lambda_{0}^{k}$ и $\Psi_{0}^{k}$ обладают дополнительными свойствами, описанньми в замечании 1.3 . Для доказательства этих свойств представим теперь $\Lambda_{0}^{k}$ и $\Psi_{0}^{k}$ в виде рядов по степеням $\mu$, подставим их в (1.7), (1.8) и вычислим коэффициенты при одинаковых степенях $\mu$. Полученная таким образом рекуррентная система краевых задач, как легко доказать с учетом простоты $\lambda_{0}^{k}$, однозначно разрешима, ее решения непрерывны 
при $\varepsilon \rightarrow 0$ и могут быть оценены равномерно по $\varepsilon$. Последние оценки позволяют построить не зависящие от $\varepsilon$ и голоморфные по $\mu$ мажоранты для $\Lambda_{0}^{k}$ и $\Psi_{0}^{k}$. Таким образом, утверждение замечания 1.3 полностью доказано.

Пусть выполнено условие (C0), и пусть

$$
a^{j}(\varepsilon)=\left(\theta_{\varepsilon}\left(s_{j}^{\varepsilon}\right)-\theta_{\varepsilon}\left(s_{j}^{\varepsilon}-\varepsilon a_{j}(\varepsilon)\right)\right) / \varepsilon, \quad b^{j}(\varepsilon)=\left(\theta_{\varepsilon}\left(s_{j}^{\varepsilon}+\varepsilon b_{j}(\varepsilon)\right)-\theta_{\varepsilon}\left(s_{j}^{\varepsilon}\right)\right) / \varepsilon .
$$

$\Phi$ ункции $a^{j}$ и $b^{j}$ описывают образ множества $\gamma_{\varepsilon, j}$ при отображении $\theta_{\varepsilon}$ : длина этого образа равна $\varepsilon\left(a^{j}+b^{j}\right)$, а его конщевые точки соответствуют углам $\left(\theta_{\varepsilon}\left(s_{j}^{\varepsilon}\right)-\varepsilon a^{j}(\varepsilon)\right)$ и $\left(\theta_{\varepsilon}\left(s_{j}^{\varepsilon}\right)+\varepsilon b^{j}(\varepsilon)\right)$. Пусть теперь выполнено и условие (C1), и пусть

$$
\begin{array}{ll}
\alpha^{j}=\alpha^{j}(\varepsilon)=\frac{a^{j}(\varepsilon)}{2 \eta(\varepsilon)}, & \beta^{j}=\beta^{j}(\varepsilon)=\frac{b^{j}(\varepsilon)}{2 \eta(\varepsilon)}, \quad \delta^{*}(\varepsilon)=\max _{j}\left|\delta^{j}(\varepsilon)\right|, \\
\alpha_{j}=\alpha_{j}(\varepsilon)=\frac{a_{j}(\varepsilon)}{2 \eta(\varepsilon)}, & \beta_{j}=\beta_{j}(\varepsilon)=\frac{b_{j}(\varepsilon)}{2 \eta(\varepsilon)}, \quad \delta_{*}(\varepsilon)=\max _{j}\left|\delta_{j}(\varepsilon)\right| .
\end{array}
$$

Заметим, что $d^{j}=\alpha^{j}+\beta^{j}$.

ЛЕмма 2.1. Пусть выполнены условия (C0) и (C1). Тогда справедливы оценки

$$
c_{1}\left(a_{j}(\varepsilon)+b_{j}(\varepsilon)\right) \leqslant a^{j}(\varepsilon)+b^{j}(\varepsilon) \leqslant c_{2}\left(a_{j}(\varepsilon)+b_{j}(\varepsilon)\right), \quad \delta^{*}(\varepsilon) \leqslant C\left(\delta_{*}(\varepsilon)+\varepsilon\right),
$$

где константа $C$ не зависит от $\varepsilon$ и $\eta$.

ДокАЗАТЕЛЬСтво. В силу теоремы Лагранжа и определения функций $a^{j}$ и $b^{j}$ имеем

$$
a^{j}(\varepsilon)+b^{j}(\varepsilon)=\theta_{\varepsilon}^{\prime}\left(M_{j, \varepsilon}^{(1)}\right)\left(a_{j}(\varepsilon)+b_{j}(\varepsilon)\right),
$$

где $M_{j, \varepsilon}^{(1)}-$ некоторая точка из интервала $\left(s_{j}^{\varepsilon}-\varepsilon a_{j}(\varepsilon), s_{j}^{\varepsilon}+\varepsilon b_{j}(\varepsilon)\right)$. Используя теперь оценку производной $\theta_{\varepsilon}^{\prime}$ из условия (С0), приходим к первому неравенству утверждения леммы.

В силу (2.1) и определения $\delta^{j}(\varepsilon)$ выводим:

$$
\begin{aligned}
\delta^{j} & =\theta_{\varepsilon}^{\prime}\left(M_{j+1, \varepsilon}^{(1)}\right)\left(\alpha_{j+1}+\beta_{j+1}\right)-\theta_{\varepsilon}^{\prime}\left(M_{j, \varepsilon}^{(1)}\right)\left(\alpha_{j}+\beta_{j}\right) \\
& =\theta_{\varepsilon}^{\prime}\left(M_{j+1, \varepsilon}^{(1)}\right) \delta_{j}+\left(\alpha_{j}+\beta_{j}\right)\left(\theta_{\varepsilon}^{\prime}\left(M_{j+1, \varepsilon}^{(1)}\right)-\theta_{\varepsilon}^{\prime}\left(M_{j, \varepsilon}^{(1)}\right)\right) .
\end{aligned}
$$

Величину $\theta_{\varepsilon}^{\prime}\left(M_{j+1, \varepsilon}^{(1)}\right)-\theta_{\varepsilon}^{\prime}\left(M_{j, \varepsilon}^{(1)}\right)$ согласно теореме Лагранжа можно представить в виде

$$
\theta_{\varepsilon}^{\prime}\left(M_{j+1, \varepsilon}^{(1)}\right)-\theta_{\varepsilon}^{\prime}\left(M_{j, \varepsilon}^{(1)}\right)=\theta_{\varepsilon}^{\prime \prime}\left(M_{j, \varepsilon}^{(2)}\right)\left(M_{j+1, \varepsilon}^{(1)}-M_{j, \varepsilon}^{(1)}\right),
$$

где точка $M_{j, \varepsilon}^{(2)}$, по определению $M_{j, \varepsilon}^{(1)}$, лежит в интервале $\left(s_{j}^{\varepsilon}-\varepsilon a_{j}(\varepsilon), s_{j+1}^{\varepsilon}+\right.$ $\left.\varepsilon b_{j+1}(\varepsilon)\right)$. В силу последнего равенства и условий $(\mathrm{C} 0)$ и $(\mathrm{C} 1)$ второе слагаемое в правой части (2.2) оценим следуюшим образом:

$$
\left|\left(\alpha_{j}+\beta_{j}\right) \theta_{\varepsilon}^{\prime \prime}\left(M_{j, \varepsilon}^{(2)}\right)\left(M_{j+1, \varepsilon}^{(1)}-M_{j, \varepsilon}^{(1)}\right)\right| \leqslant C\left(s_{j+1}^{\varepsilon}-s_{j}^{\varepsilon}+\varepsilon\left(b_{j+1}+a_{j}\right)\right) \leqslant C \varepsilon,
$$

где константы $C$ не зависят от $\varepsilon, \eta$ и $j$. Здесь мы также применили легко доказываемое соотношение

$$
c_{1}\left|s_{j+1}^{\varepsilon}-s_{j}^{\varepsilon}\right| \leqslant \theta_{\varepsilon}\left(s_{j+1}^{\varepsilon}\right)-\theta_{\varepsilon}\left(s_{j}^{\varepsilon}\right)=\varepsilon \pi .
$$

Подставляя $(2.3)$ в $(2.2)$ и используя оценку величины $\theta_{\varepsilon}^{\prime}\left(M_{j+1, \varepsilon}^{(1)}\right)$, с помощю условия (C0) получим второе неравенство утверждения леммы. Лемма доказана.

Из доказанной леммы очевидным образом вытекает 
СлЕДСТВИЕ 2.1. В условиях теоремы 1.4 верно соотношение

$$
\delta^{*}(\varepsilon)=o\left(\varepsilon^{1 / 2}(A+\mu)^{-1}\right) .
$$

СлЕДСТВИЕ 2.2. Функиия $\delta^{*}(\varepsilon)$ ограничена.

ДоКАЗАТЕЛЬСТВО. Из леммы 2.1 и условия $(\mathrm{C} 1)$ имеем

$$
\left|d^{j}(\varepsilon)\right| \leqslant c_{2} \frac{a^{j}(\varepsilon)+b^{j}(\varepsilon)}{2 \eta(\varepsilon)} \leqslant 1,
$$

откуда и следует ограниченность $\delta^{*}$. Следствие доказано.

ДОКАЗАТЕЛЬСТвО ТЕОРЕМЫ 1.4. Сходимость собственных значений возмущенной задачи к собственным значениям задачи (1.6) при выполнении условий $(\mathrm{C} 0),(\mathrm{C} 1)$ и равенства $(1.5)$ нетрудно доказать, используя результаты и методику работ [2], [6], [8].

Поясним схему доказательства асимптотик. Вначале формально построим асимптотики собственных значений и собственных функций возмущенной задачи. Затем проведем строгое обоснование формальных асимптотических разложений, т.е. докажем, что формально построенные асимптотические разложения действительно являются асимптотиками собственных элементов возмущенной задачи. При формальном построении будем предполагать лишь ограниченность функции $\delta^{*}(\varepsilon)$ (см. следствие 2.2), а равенством (1.9) воспользуемся только при обосновании асимптотик для оценки остатков.

Формальное построение асимптотических разложений подробно проведем для случая простого предельного собственного значения. Такой выбор обусловлен, с одной стороны, желанием избежать излишней громоздкости изложения, с другой стороны, построение не зависит, по существу, от кратности предельного собственного значения. Далее поясним построение в случае кратного предельного собственного значения.

Перейдем непосредственно к построению асимптотик. Пусть $\lambda_{0}-$ простое собственное значение задачи (1.6), $\lambda_{\varepsilon}$ - сходящееся к $\lambda_{0}$ собственное значение возмушенной задачи, $\psi_{\varepsilon}$ и $\psi_{0}$ - соответствуюшие собственные функции. Вначале продемонстрируем саму схему построения и формально получим первые члены асимптотик. Асимптотику собственного значения будем строить в виде

$$
\lambda_{\varepsilon}=\Lambda_{0}(\mu, \varepsilon)+\varepsilon \Lambda_{1}(\mu, \varepsilon) .
$$

Асимптотика $\psi_{\varepsilon}$ строится на основе комбинации метода согласования асимптотических разложений [32], метода составных разложений [33] и метода многих масштабов [34]. Эта асимптотика будет получена как сумма трех разложений: внешнего разложения, пограничного слоя и внутреннего разложения. Внешнее разложение построим следуюшим образом:

$$
\psi_{\varepsilon}^{\mathrm{ex}}(x, \mu)=\Psi_{0}(x, \mu, \varepsilon)+\varepsilon \Psi_{1}(x, \mu, \varepsilon) .
$$

Пограничный слой построим с использованием метода составных разложений в виде

$$
\psi_{\varepsilon}^{\mathrm{bl}}(\xi, s, \mu)=\varepsilon v_{1}(\xi, s, \mu, \varepsilon)+\varepsilon^{2} v_{2}(\xi, s, \mu, \varepsilon),
$$


где $\xi=\left(\xi_{1}, \xi_{2}\right)=\left(\left(\theta_{\varepsilon}(s)-\theta_{\varepsilon}\left(s_{0}^{\varepsilon}\right)\right) / \varepsilon, \tau \theta_{\varepsilon}^{\prime}(s) / \varepsilon\right)$ - "растянутые" переменные. Здесь $(s, \tau)$ - локальные переменные, введенные в окрестности границы $\partial \Omega, \tau$ - расстояние от точки до границы $\partial \Omega$, измеренное в направлении внутренней нормали. Данньй выбор переменных $\xi$ поясним ниже, в замечании 2.1 .

Внутреннее разложение построим на основе метода согласования асимптотических разложений в малых окрестностях точек $x_{j}^{\varepsilon}$ в виде

$$
\psi_{\varepsilon}^{\text {in }, j}\left(\varsigma^{j}, s, \mu\right)=w_{0,0}^{(j)}\left(\varsigma^{j}, s, \mu, \varepsilon\right)+\varepsilon w_{1,0}^{(j)}\left(\varsigma^{j}, s, \mu, \varepsilon\right)
$$

где $\varsigma^{j}=\left(\varsigma_{1}^{j}, \varsigma_{2}^{j}\right)=\left(\left(\xi_{1}-\pi j\right) \eta^{-1}, \xi_{2} \eta^{-1}\right)$.

Целью формального построения является определение вида функций $\Lambda_{i}, \Psi_{i}, v_{i}$ и $w_{i, 0}$.

Уравнения для функций $\Psi_{0}$ и $\Psi_{1}$ получаются стандартной подстановкой (2.4) и (2.5) в уравнение (1.1) с последуюшим выписыванием коэффициентов при одинаковых степенях $\varepsilon$. Такая процедура приводит к уравнению (1.7) для функции $\Psi_{0}$ с $\Lambda_{0}^{k}=\Lambda_{0}$ и следуюшему уравнению для $\Psi_{1}$ :

$$
\left(\Delta+\Lambda_{0}\right) \Psi_{1}=-\Lambda_{1} \Psi_{0}, \quad x \in \Omega
$$

Граничные условия для функций $\Psi_{0}$ и $\Psi_{1}$ будут определены ниже при построении коэффициентов пограничного слоя и внутреннего разложения.

Определим теперь функции $v_{i}$. Вначале получим задачи для этих функций, для чего необходимо переписать оператор Лапласа в переменных $(s, \tau)$ :

$$
\Delta_{x}=\frac{1}{P}\left(\frac{\partial}{\partial \tau}\left(P \frac{\partial}{\partial \tau}\right)+\frac{\partial}{\partial s}\left(\frac{1}{P} \frac{\partial}{\partial s}\right)\right), \quad P=P(s, \tau)=1+\tau K(s),
$$

$K=K(s)=\left(\mathbf{r}^{\prime \prime}(s), \nu(s)\right)_{\mathbb{R}^{2}}, \nu=\nu(s), \mathbf{r}(s)$ - двумерная вектор-функция, задающая кривую $\partial \Omega, K \in C^{\infty}(\partial \Omega)$. Теперь подставим $(2.4),(2.5)$ в уравнение $(1.1)$ с учетом приведенного выше представления для оператора Лапласа, перейдем к переменным $\xi$ и выпишем коэффициенты при старших степенях $\varepsilon$. Тогда для функций $v_{1}$ и $v_{2}$ получим уравнения

$$
\begin{gathered}
\Delta_{\xi} v_{1}=0, \quad \xi_{2}>0 \\
\Delta_{\xi} v_{2}=-\frac{\theta_{\varepsilon}^{\prime \prime}}{\left(\theta_{\varepsilon}^{\prime}\right)^{2}}\left(\frac{\partial}{\partial \xi_{1}}+2 \xi_{2} \frac{\partial^{2}}{\partial \xi_{1} \partial \xi_{2}}\right) v_{1} \\
-\frac{K}{\theta_{\varepsilon}^{\prime}}\left(\frac{\partial}{\partial \xi_{2}}-2 \xi_{2} \frac{\partial^{2}}{\partial \xi_{1}^{2}}\right) v_{1}-\frac{2}{\theta_{\varepsilon}^{\prime}} \frac{\partial^{2} v_{1}}{\partial \xi_{1} \partial s}, \quad \xi_{2}>0 .
\end{gathered}
$$

Согласно методу составных разложений сумма функций $\psi_{\varepsilon}^{\mathrm{ex}}$ и $\psi_{\varepsilon}^{\mathrm{bl}}$ удовлетворяет однородному граничному условию Неймана всюду на $\partial \Omega$, за исключением точек $x_{j}^{\varepsilon}$ :

$$
\frac{\partial}{\partial \nu} \psi_{\varepsilon}^{\mathrm{ex}}-\frac{\partial}{\partial \tau} \psi_{\varepsilon}^{\mathrm{bl}}=0, \quad x \in \partial \Omega, \quad x \neq x_{j}^{\varepsilon}
$$


Перепишем теперь второе слагаемое последнего равенства в переменных $\xi$ и заменим функции $\psi_{\varepsilon}^{\mathrm{ex}}$ и $\psi_{\varepsilon}^{\mathrm{bl}}$ на правые части равенств $(2.5)$ и $(2.6)$, после чего вычислим коэффициенты при старших степенях $\varepsilon$, которые приравняем нулю. В результате получим граничные условия для функций $v_{i}$ :

$$
\begin{aligned}
& \frac{\partial v_{1}}{\partial \xi_{2}}=\frac{1}{\theta_{\varepsilon}^{\prime}} \Psi_{0}^{\nu}, \quad \xi \in \Gamma^{0}, \\
& \frac{\partial v_{2}}{\partial \xi_{2}}=\frac{1}{\theta_{\varepsilon}^{\prime}} \Psi_{1}^{\nu}, \quad \xi \in \Gamma^{0},
\end{aligned}
$$

где $\Gamma^{0}=\left\{\xi: \xi_{2}=0, \xi_{1} \neq \varepsilon \pi j, j \in \mathbb{Z}\right\}$

$$
\Psi_{i}^{\nu}=\Psi_{i}^{\nu}(s, \mu, \varepsilon)=\frac{\partial}{\partial \nu} \Psi_{i}(x, \mu, \varepsilon), \quad x \in \partial \Omega .
$$

ЗАмЕчАниЕ 2.1. Из определения множества $\Gamma^{0}$ следует, что для функций $v_{i}$ мы получили задачу с периодической структурой по переменной $\xi_{1}$, что по существу будет использовано при решении краевых задач (2.9)-(2.12). Как нетрудно убедиться, периодичность $\Gamma^{0}$ является прямым следствием условия $(\mathrm{C} 0)$, а именно равенства $\theta_{\varepsilon}\left(s_{j}^{\varepsilon}\right)=\theta_{\varepsilon}\left(s_{0}^{\varepsilon}\right)+\varepsilon \pi j$, и приведенного вьше определения переменной $\xi_{1}$, что и объясняет указанный выбор переменной $\xi_{1}$. Переменная $\xi_{2}$ была выбрана так, чтобы получить уравнения Пуассона для функций $v_{1}$ и $v_{2}$.

Согласно методу составных разложений будем искать экспоненциально убьвающие при $\xi_{2} \rightarrow+\infty$ решения задач $(2.9),(2.11)$ и $(2.10),(2.12)$. При построении пограничного слоя мы также используем и метод многих масштабов: роль "медленного" времени играет переменная $s$. Обратим также внимание на то обстоятельство, что пограничный слой "контролирует" только граничное условие Неймана, именно поэтому мы перешли к формальному пределу при $\eta \rightarrow 0$ при выводе граничного условия (2.11). Казалось бы, можно добиться выполнения и граничного условия Дирихле подходяшим выбором функций $v_{1}$ и $v_{2}$. Однако такой путь приводит к неразрешимым задачам для функций $v_{1}$ и $v_{2}$.

Пусть $\mathscr{V}_{0}$ - пространство $\pi$-периодических по переменной $\xi_{1}$ функций из $C^{\infty}(\{\xi$ : $\left.\left.\xi_{2}>0\right\} \cup \Gamma^{0}\right)$, экспоненциально убывающих вместе со всеми своими производными при $\xi_{2} \rightarrow+\infty$ равномерно по $\xi_{1}$.

Построим решение задачи (2.9), (2.11). Заметим, что переменная $s$ в эту задачу входит как параметр. Рассмотрим функцию

$$
X(\xi)=\operatorname{Re} \ln \sin z+\ln 2-\xi_{2},
$$

где $z=\xi_{1}+i \xi_{2}-$ комплексная переменная. Непосредственными вычислениями проверим, что $X \in \mathscr{V}_{0}$ - четная по $\xi_{1}$ гармоническая функция, удовлетворяюшая граничному условию

$$
\frac{\partial X}{\partial \xi_{2}}=-1, \quad \xi \in \Gamma^{0},
$$

для которой справедливо представление

$$
X(\xi)=\ln \rho+\ln 2-\xi_{2}+\widetilde{X}(\xi), \quad \widetilde{X}(\xi)=O\left(\rho^{2}\right), \quad \xi \rightarrow \xi^{(j)},
$$


где $\rho=\left|\xi-\xi^{(j)}\right|, \quad \xi^{(j)}=(\pi j, 0), j \in \mathbb{Z}, \widetilde{X}(\xi) \in C^{\infty}\left(\left\{\xi: \xi_{2} \geqslant 0\right\}\right)$. Учитывая все перечисленные факты, заключаем, что функция $v_{1}$ имеет следующий вид:

$$
v_{1}(\xi, s, \mu)=-\frac{1}{\theta_{\varepsilon}^{\prime}(s)} \Psi_{0}^{\nu}(s, \mu, \varepsilon) X(\xi) .
$$

Решение задачи $(2.10),(2.12)$ также можно построить в явном виде. Прямыми вычислениями проверяем, что функция

$$
\tilde{v}_{2}=\frac{\Psi_{0}^{\nu}}{2\left(\theta_{\varepsilon}^{\prime}\right)^{2}} \xi_{2}^{2}\left(\frac{\theta_{\varepsilon}^{\prime \prime}}{\theta_{\varepsilon}^{\prime}} \frac{\partial X}{\partial \xi_{1}}+K \frac{\partial X}{\partial \xi_{2}}\right)-\frac{1}{\theta_{\varepsilon}^{\prime}}\left(\frac{\Psi_{0}^{\nu}}{\theta_{\varepsilon}^{\prime}}\right)^{\prime} \int_{\xi_{2}}^{+\infty} t \frac{\partial}{\partial \xi_{1}} X\left(\xi_{1}, t\right) d t
$$

является решением уравнения (2.10), удовлетворяющим однородному граничному условию Неймана на $\Gamma^{0}$. Функция $\tilde{v}_{2} \in \mathscr{V}_{0}$ имеет следующую (дифференцируемую) асимптотику при $\xi \rightarrow \xi^{(j)}$ :

$$
\tilde{v}_{2}=O(\rho \ln \rho) .
$$

Учитывая все перечисленные свойства функций $\tilde{v}_{2}$ и $X$, приходим к выражению для функции $v_{2}$ :

$$
v_{2}(\xi, s, \mu, \varepsilon)=\tilde{v}_{2}(\xi, s, \mu, \varepsilon)-\frac{1}{\theta_{\varepsilon}^{\prime}(s)} \Psi_{1}^{\nu}(s, \mu, \varepsilon) X(\xi) .
$$

Как следует из (2.14)-(2.16), функции $v_{i}$ имеют логарифмические особенности в окрестностях точек $\xi^{(j)}$, или, что то же самое, в окрестности точек $x_{j}^{\varepsilon}$. Кроме того, сумма внешнего разложения и пограничного слоя не удовлетворяет (даже асимптотически) граничному условию Дирихле на $\gamma_{\varepsilon}$. Поэтому в окрестности точек $x_{j}^{\varepsilon}$ вводятся новые "растянутые" переменные $\varsigma^{j}$, а асимптотика собственной функции

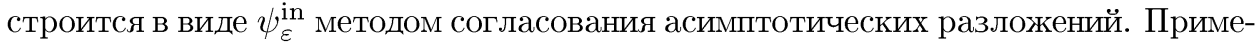
нение термина "растянутые" к переменным $\varsigma^{j}$ оправдано, так как в силу равенства (1.5) функция $\eta$ имеет следуюший вид:

$$
\eta(\varepsilon)=\exp \left(-\frac{1}{\varepsilon(A+\mu(\varepsilon))}\right)
$$

где $\mu(\varepsilon)$ - такое, как в утверждении доказываемой теоремы. Таким образом, функция $\eta(\varepsilon)$ экспоненциально мала по сравнению с $\varepsilon$.

Вначале проведем согласование асимптотических разложений в окрестности точки $x_{j}^{\varepsilon}$. Для краткости обозначим $\varsigma=\varsigma^{j}$. Ясно, что при $\tau \rightarrow 0$ верны асимптотики

$$
\psi_{\varepsilon}^{\operatorname{ex}}(x)=\sum_{i=0}^{1} \varepsilon^{i}\left(\Psi_{i}^{D}(s, \mu, \varepsilon)-\tau \Psi_{i}^{\nu}(s, \mu, \varepsilon)\right)+O\left(\tau^{2}\right),
$$

где $\Psi_{i}^{D}$ - значения функций $\Psi_{i}$ при $x \in \partial \Omega$, а переменная $s$ меняется в малой окрестности значения $s_{j}^{\varepsilon}$. Учитывая асимптотики $(2.14),(2.15)$ и определения функций $v_{i}$, получаем, что при $\xi \rightarrow \xi^{(j)}$

$$
\psi_{\varepsilon}^{\mathrm{bl}}(\xi, s, \mu)=-\varepsilon\left(\ln \rho+\ln 2-\xi_{2}\right) \sum_{i=0}^{1} \varepsilon^{i} \frac{\Psi_{i}^{\nu}(s, \mu, \varepsilon)}{\theta_{\varepsilon}^{\prime}(s)}+O(\rho \ln \rho) .
$$


Перепишем теперь асимптотики (2.18), (2.19) в переменных $\varsigma$ и учтем, что в силу (2.17) выполнено равенство $\varepsilon \ln \eta(\varepsilon)=-(A+\mu)^{-1}$. Тогда получим, что при $\frac{1}{4} \eta^{1 / 4}<\rho<\frac{3}{4} \eta^{1 / 4}$ (или, что то же самое, при $\frac{1}{4} \eta^{-3 / 4}<|\varsigma|<\frac{3}{4} \eta^{-3 / 4}$ )

$$
\begin{gathered}
\psi_{\varepsilon}^{\mathrm{ex}, \mu}(x)+\psi_{\varepsilon}^{\mathrm{bl}}(\xi, s, \mu)=W_{0,0}(s, \mu, \varepsilon)+\varepsilon W_{1,0}(\varsigma, s, \mu, \varepsilon)+O\left(\varepsilon^{2} \ln |\varsigma|\right), \\
W_{0,0}(s, \mu, \varepsilon)=\Psi_{0}^{D}(s, \mu, \varepsilon)+\frac{\Psi_{0}^{\nu}(s, \mu, \varepsilon)}{(A+\mu) \theta_{\varepsilon}^{\prime}(s)} \\
W_{1,0}(\varsigma, s, \mu, \varepsilon)=-\frac{\Psi_{0}^{\nu}(s, \mu, \varepsilon)}{\theta_{\varepsilon}^{\prime}(s)}(\ln |\varsigma|+\ln 2)+\Psi_{1}^{D}(s, \mu)+\frac{\Psi_{1}^{\nu}(s, \mu, \varepsilon)}{(A+\mu) \theta_{\varepsilon}^{\prime}(s)} .
\end{gathered}
$$

В силу метода согласования асимптотических разложений из (2.20) вытекает, что функции $w_{i, 0}^{(j)}$ имеют следуюшие асимптотики на бесконечности:

$$
w_{i, 0}^{(j)}=W_{i, 0}+o(1), \quad \varsigma \rightarrow \infty .
$$

Задачи для функций $w_{i, 0}$ получаем стандартной подстановкой $(2.4)$ и $(2.7)$ в краевую задачу (1.1), (1.2) и выписыванием коэффициентов при старших степенях $\varepsilon$ и $\eta$ :

$$
\begin{aligned}
\Delta_{\varsigma} w_{i, 0}^{(j)} & =0, & & \varsigma_{2}>0, \\
w_{i, 0}^{(j)} & =0, & & \varsigma \in \gamma_{j}^{1}, \\
\frac{\partial}{\partial \varsigma_{2}} w_{i, 0}^{(j)} & =0, & & \varsigma \in \Gamma_{j}^{1} .
\end{aligned}
$$

Здесь $\gamma_{j}^{1}$ - интервал $\left(-2 \alpha^{j}, 2 \beta^{j}\right)$ на оси $O \varsigma_{1}$, a $\Gamma_{j}^{1}-$ дополнение замыкания $\gamma_{j}^{1}$ до прямой $\varsigma_{2}=0$.

Задача (2.24) не имеет ограниченных на бесконечности нетривиальных решений, а потому ввиду $(2.21),(2.23)$ имеем

$$
w_{0,0}^{(j)}=0 .
$$

Из этого равенства и асимптотики (2.21), (2.23) вытекает граничное условие (1.8) для функции $\Psi_{0}$. Собственные элементы $\Lambda_{0}$ и $\Psi_{0}$ удовлетворяют лемме 1.1. В силу гладкости границы области и функции $\theta_{\varepsilon}^{\prime}(s)$ функция $\Psi_{0}$ бесконечно дифференцируема по переменным $x$.

Определим теперь функцию $w_{1,0}$. Пусть

$$
Y^{(j)}(\varsigma, \varepsilon)=\operatorname{Re} \ln \left(y+\sqrt{y^{2}-1}\right)
$$

где $y=\left(\varsigma_{1}+i \varsigma_{2}+\alpha^{j}-\beta^{j}\right) /\left(\alpha^{j}+\beta^{j}\right)-$ комплексная переменная. Легко убедиться, что $Y^{(j)} \in \mathscr{W}$, где обозначено

$$
\mathscr{W} \equiv C^{\infty}\left(\left\{\varsigma: \varsigma_{2} \geqslant 0, \varsigma \neq\left(-2 \alpha^{j}, 0\right), \varsigma \neq\left(2 \beta^{j}, 0\right)\right\}\right) \cap H^{1}\left(\left\{\varsigma: \varsigma_{2}>0,|\varsigma|<5\right\}\right) .
$$

Функция $Y^{(j)}$ есть решение задачи $(2.24)$, имеющее на бесконечности асимптотику:

$$
Y^{(j)}=\ln |\varsigma|+\ln 2-\ln \left(\alpha^{j}+\beta^{j}\right)+\left(\alpha^{j}-\beta^{j}\right) \varsigma_{1}|\varsigma|^{-2}+O\left(|\varsigma|^{-2}\right), \quad \varsigma \rightarrow \infty .
$$


В силу перечисленных свойств $Y^{(j)}$ функция $w_{1,0}^{(j)}$ имеет следуюший вид:

$$
w_{1,0}^{(j)}(\varsigma, s, \mu, \varepsilon)=-\frac{\Psi_{0}^{\nu}(s, \mu, \varepsilon)}{\theta_{\varepsilon}^{\prime}(s)} Y^{(j)}(\varsigma, \varepsilon) \text {. }
$$

Ясно, что $w_{1,0}^{(j)} \in \mathscr{W}$. Выпишем теперь асимптотику функции $w_{1,0}^{(j)}$ на бесконечности (см. (2.26)) и сравним ее с $(2.22),(2.23)$. В результате такого сравнения приходим к равенству

$$
\frac{\Psi_{0}^{\nu}(s, \mu, \varepsilon)}{\theta_{\varepsilon}^{\prime}(s)} \ln \left(\alpha^{j}(\varepsilon)+\beta^{j}(\varepsilon)\right)=\Psi_{1}^{D}(s, \mu, \varepsilon)+\frac{\Psi_{1}^{\nu}(s, \mu, \varepsilon)}{(A+\mu) \theta_{\varepsilon}^{\prime}(s)},
$$

фактически являющемуся граничным условием для функции $\Psi_{1}$. Необходимо лишш корректно определить правую часть граничного условия, которая бы учитывала то обстоятельство, что, вообще говоря, величины $\ln \left(\alpha^{j}+\beta^{j}\right)$ зависят от номера $j$ и параметра $\varepsilon$. Как уже было отмечено выше, переменная $s$ в равенстве $(2.27)$ меняется в малой (порядка $\left.O\left(\varepsilon \eta^{1 / 4}\right)\right)$ окрестности точки $s_{j}^{\varepsilon}$. Следовательно, для выполнения равенства (2.27) достаточно построить функцию, которая была бы равна $\alpha^{j}+\beta^{j}$ в указанных окрестностях точек $s_{j}^{\varepsilon}$, и заменить затем $\alpha^{j}+\beta^{j}$ в $(2.27)$ на эту функцию. Функция $g_{\varepsilon}(\theta)$, как несложно убедиться, бесконечно дифференцируема по $\theta$ и равна $d^{j}=\alpha^{j}+\beta^{j}$ при $\left|\theta-\theta_{\varepsilon}\left(s_{j}^{\varepsilon}\right)\right| \leqslant \varepsilon \pi / 4$. Поэтому в качестве функции, на которую заменяется сумма $\alpha^{j}+\beta^{j}$ в $(2.27)$, мы возьмем $g_{\varepsilon}\left(\theta_{\varepsilon}(s)\right)$, откуда немедленно получим граничное условие для $\Psi_{1}$ :

$$
\left(\frac{\partial}{\partial \nu}+(A+\mu) \theta_{\varepsilon}^{\prime}(s)\right) \Psi_{1}=(A+\mu) \Psi_{0}^{\nu}(s, \mu, \varepsilon) \ln g_{\varepsilon}\left(\theta_{\varepsilon}(s)\right), \quad x \in \partial \Omega .
$$

Теперь учтем, что $\Psi_{0}^{\nu}=-(A+\mu) \theta_{\varepsilon}^{\prime} \Psi_{0}^{D}$, и окончательно получим

$$
\left(\frac{\partial}{\partial \nu}+(A+\mu) \theta_{\varepsilon}^{\prime}(s)\right) \Psi_{1}=-(A+\mu)^{2} \Psi_{0}^{D}(s, \mu, \varepsilon) \theta_{\varepsilon}^{\prime}(s) \ln g_{\varepsilon}\left(\theta_{\varepsilon}(s)\right), \quad x \in \partial \Omega .
$$

Задача (2.8), (2.28) разрешима при подходяшем выборе $\Lambda_{1}$. Условие разрешимости получается стандартно, умножением уравнения (2.8) на $\Psi_{0}$ и интегрированием по частям с учетом граничного условия (2.28). Принимая во внимание нормировку функции $\Psi_{0}$, из указанного условия разрешимости получаем формулу (1.11) для первого члена асимптотики. Из леммы 2.1 , условия $(\mathrm{C} 1)$ и определения $g_{\varepsilon}$ следует оценка

$$
c_{1} c_{4} / 2 \leqslant g_{\varepsilon}(\theta) \leqslant 1
$$

откуда в силу формулы (1.11) вытекает неположительность $\Lambda_{1}$. Утверждаемая голоморфность $\Lambda_{1}$ по $\mu$ является следствием соответствуюших свойств $\Psi_{0}$, ограниченности $\theta_{\varepsilon}^{\prime}$ и $g_{\varepsilon}(\theta)$ и оценки нормы $\left\|\Psi_{0}\right\|_{L_{2}(\partial \Omega)}$ через $\left\|\Psi_{0}\right\|_{H^{1}(\Omega)}$.

Функция $\Psi_{1}$ определена с точностью до слагаемого $C \Psi_{0}, C=$ const. Выбираем функцию $\Psi_{1}$, считая ее ортогональной $\Psi_{0}$ в $L_{2}(\Omega)$. Функция $\ln g_{\varepsilon}\left(\theta_{\varepsilon}(s)\right)$ гладкая, поэтому нетрудно показать, что $\Psi_{1} \in C^{\infty}(\bar{\Omega})$. Кроме того, функция $\Psi_{1}$ голоморфна по $\mu$ в норме $H^{1}(\Omega)$ для каждого фиксированного значения $\varepsilon$ [35]. Используя однократность $\lambda_{0}$, нетрудно доказать, что коэффициенты разложения $\Psi_{1}$ в ряд Тейлора по степеням $\mu$ непрерывны по $\varepsilon \rightarrow 0$, а функция $\Psi_{1}$ мажорируется голоморфной по $\mu$ функцией, не зависящей от $\varepsilon$. 
Проведенное построение позволило определить первые члены асимптотических разложений $\lambda_{\varepsilon}$ и $\psi_{\varepsilon}$ (на формальном уровне, разумеется). Необходимо теперь доказать, что формально построенные асимптотики действительно являются асимптотиками $\lambda_{\varepsilon}$ и $\psi_{\varepsilon}$. Для того чтобы провести такое обоснование, предварительно докажем, что построенные асимптотики являются формальным асимптотическим решением возмущенной задачи, т. е. удовлетворяют ей с точностью до достаточно малой невязки. Именно доказательство последнего утверждения будет нашей целью на данном этапе. Чтобы провести это доказательство и обеспечить необходимую малость невязки, нужно построить дополнительные члены в асимптотических разложениях для $\lambda_{\varepsilon}$ и $\psi_{\varepsilon}$.

Во внешнем разложении необходимо построить одно дополнительное слагаемое:

$$
\psi_{\varepsilon}^{\mathrm{ex}}(x, \mu)=\Psi_{0}(x, \mu, \varepsilon)+\varepsilon \Psi_{1}(x, \mu, \varepsilon)+\varepsilon^{2} \Psi_{2}(x, \mu, \varepsilon) .
$$

В пограничном слое необходимо построить еще два члена; в результате пограничный слой выглядит следующим образом:

$$
\psi_{\varepsilon}^{\mathrm{bl}}(\xi, s, \mu)=\sum_{i=1}^{4} \varepsilon^{i} v_{i}(\xi, s, \mu, \varepsilon)
$$

С учетом равенства $w_{0,0}^{(j)}=0$ и дополнительных членов внутреннее разложение имеет следуюший вид:

$$
\psi_{\varepsilon}^{\text {in }, j}\left(\varsigma^{j}, s, \mu\right)=\sum_{i=1}^{3} \varepsilon^{i} w_{i, 0}^{(j)}\left(\varsigma^{j}, s, \mu, \varepsilon\right)+\eta \sum_{i=1}^{4} \varepsilon^{i} w_{i, 1}^{(j)}\left(\varsigma^{j}, s, \mu, \varepsilon\right) .
$$

Во внешнем разложении (2.30) первые члены известны, осталось определить лишь функцию $\Psi_{2}$. Эта функция будет использована далее только для того, чтобы провести согласование дополнительных членов внутреннего разложения. Как и ранее, согласование будет влиять лишь на вид граничного условия для $\Psi_{2}$, а потому имеет место определенная свобода в выборе уравнения для $\Psi_{2}$, так как конкретный вид этого уравнения не оказывает существенного влияния на оценку невязки. Уравнение для $\Psi_{2}$ мы выбираем так, чтобы, во-первых, гарантировать его разрешимость и, во-вторых, упростить дальнейшие вычисления. Эти две цели достигаются за счет следуюшего выбора:

$$
(\Delta-1) \Psi_{2}=-\Lambda_{1} \Psi_{1}, \quad x \in \Omega \text {. }
$$

Дополнительные члены пограничного слоя зададим следуюшим образом:

$$
\begin{aligned}
v_{3}= & \frac{\Psi_{1}^{\nu}}{2\left(\theta_{\varepsilon}^{\prime}\right)^{2}} \xi_{2}^{2}\left(\frac{\theta_{\varepsilon}^{\prime \prime}}{\theta_{\varepsilon}^{\prime}} \frac{\partial X}{\partial \xi_{1}}+K \frac{\partial X}{\partial \xi_{2}}\right)-\frac{1}{\theta_{\varepsilon}^{\prime}}\left(\frac{\Psi_{1}^{\nu}}{\theta_{\varepsilon}^{\prime}}\right)^{\prime} \int_{\xi_{2}}^{+\infty} t \frac{\partial}{\partial \xi_{1}} X\left(\xi_{1}, t\right) d t \\
& +l \int_{\xi_{2}}^{+\infty} t X\left(\xi_{1}, t\right) d t-\frac{1}{\theta_{\varepsilon}^{\prime}} \Psi_{2}^{\nu} X \\
v_{4}= & \frac{\Psi_{2}^{\nu}}{2\left(\theta_{\varepsilon}^{\prime}\right)^{2}} \xi_{2}^{2}\left(\frac{\theta_{\varepsilon}^{\prime \prime}}{\theta_{\varepsilon}^{\prime}} \frac{\partial X}{\partial \xi_{1}}+K \frac{\partial X}{\partial \xi_{2}}\right)-\frac{1}{\theta_{\varepsilon}^{\prime}}\left(\frac{\Psi_{2}^{\nu}}{\theta_{\varepsilon}^{\prime}}\right)^{\prime} \int_{\xi_{2}}^{+\infty} t \frac{\partial}{\partial \xi_{1}} X\left(\xi_{1}, t\right) d t
\end{aligned}
$$


где $l=l(s, \mu, \varepsilon)$ - некоторая функция, которая будет определена ниже, $\Psi_{2}^{\nu}$ - значение нормальной производной $\Psi_{2}$ на гранище $\partial \Omega$. Легко убедиться в том, что $v_{3}, v_{4} \in \mathscr{V}_{0}$.

Для того чтобы провести согласование асимптотических разложений и определить внутреннее разложение, необходимы следуюшие дифференцируемые асимптотики $(\rho \rightarrow 0)$ :

$$
\begin{aligned}
v_{1}(\xi, s, \mu, \varepsilon)= & -\frac{\Psi_{0}^{\nu}}{\theta_{\varepsilon}^{\prime}}\left(\ln \rho+\ln 2-\xi_{2}\right)+O\left(\rho^{2}\right), \\
v_{2}(\xi, s, \mu, \varepsilon)=- & \frac{\Psi_{1}^{\nu}}{\theta_{\varepsilon}^{\prime}}\left(\ln \rho+\ln 2-\xi_{2}\right) \\
& +V_{\varepsilon}\left(\xi-\xi^{(j)}, s, \Psi_{0}^{\nu}(s, \mu, \varepsilon)\right)+O\left(\rho^{2}\right), \\
v_{3}(\xi, s, \mu, \varepsilon)=- & \frac{\Psi_{2}^{\nu}}{\theta_{\varepsilon}^{\prime}}\left(\ln \rho+\ln 2-\xi_{2}\right)-\frac{\zeta(3)}{4} l(s, \mu, \varepsilon) \\
& +V_{\varepsilon}\left(\xi-\xi^{(j)}, s, \Psi_{1}^{\nu}(s, \mu, \varepsilon)\right)+O\left(\rho^{2} \ln \rho\right), \\
v_{4}(\xi, s, \mu, \varepsilon)= & V_{\varepsilon}\left(\xi-\xi^{(j)}, s, \Psi_{2}^{\nu}(s, \mu, \varepsilon)\right)+O\left(\rho^{2}\right),
\end{aligned}
$$

где $\zeta(t)$ - дзета-функция Римана и

$$
\begin{aligned}
V_{\varepsilon}(\xi, s, \Psi(s))= & \frac{\Psi(s)}{2\left(\theta_{\varepsilon}^{\prime}(s)\right)^{2}} \frac{\xi_{2}^{2}}{|\xi|^{2}}\left(\frac{\theta_{\varepsilon}^{\prime \prime}(s)}{\theta_{\varepsilon}^{\prime}(s)} \xi_{1}+K(s) \xi_{2}\right) \\
& +\frac{1}{\theta_{\varepsilon}^{\prime}(s)}\left(\frac{\Psi(s)}{\theta_{\varepsilon}^{\prime}(s)}\right)^{\prime} \xi_{1}(\ln |\xi|+\ln 2-1) .
\end{aligned}
$$

Коэффициенты внешнего разложения в окрестности границы $\partial \Omega$ удовлетворяют соотношениям

$$
\Psi_{i}=\Psi_{i}^{D}-\tau \Psi_{i}^{\nu}+O\left(\tau^{2}\right), \quad \tau \rightarrow 0, \quad i=0,1,2 .
$$

Переписьвая теперь приведенные асимптотики функций $v_{i}$ и $\Psi_{j}$ в переменных $\varsigma$, с учетом равенства (2.17) получаем, что при $\frac{1}{4} \eta^{1 / 4}<\rho<\frac{3}{4} \eta^{1 / 4}\left(\frac{1}{4} \eta^{-3 / 4}<|\varsigma|<\right.$ $\left.\frac{3}{4} \eta^{-3 / 4}\right)$ справедливо равенство

$$
\begin{aligned}
& \psi_{\varepsilon}^{\mathrm{ex}}(x, \mu)+\psi_{\varepsilon}^{\mathrm{bl}}(\xi, s, \mu)=\sum_{k=1}^{3} \varepsilon^{k} W_{k, 0}(\varsigma, s, \mu, \varepsilon) \\
& +\eta \sum_{k=1}^{4} \varepsilon^{k} W_{k, 1}(\varsigma, s, \mu, \varepsilon)+O\left(\eta^{2}|\varsigma|^{2} \ln |\varsigma|\right), \\
& W_{2,0}=-\frac{\Psi_{1}^{\nu}}{\theta_{\varepsilon}^{\prime}}(\ln |\varsigma|+\ln 2)+\Psi_{2}^{D}+\frac{\Psi_{2}^{\nu}}{(A+\mu) \theta_{\varepsilon}^{\prime}}, \\
& W_{3,0}=-\frac{\Psi_{2}^{\nu}}{\theta_{\varepsilon}^{\prime}}(\ln |\varsigma|+\ln 2)-\frac{\zeta(3)}{4} l, \\
& W_{1,1}=-\frac{1}{(A+\mu) \theta_{\varepsilon}^{\prime}}\left(\frac{\Psi_{0}^{\nu}}{\theta_{\varepsilon}^{\prime}}\right)^{\prime} \varsigma_{1}, \\
& W_{k, 1}=V_{\varepsilon}\left(\varsigma, s, \Psi_{k-2}^{\nu}\right)-\frac{1}{(A+\mu) \theta_{\varepsilon}^{\prime}}\left(\frac{\Psi_{k-1}^{\nu}}{\theta_{\varepsilon}^{\prime}}\right)^{\prime} \varsigma_{1}, \quad k=2,3, \\
& W_{4,1}=V_{\varepsilon}\left(\varsigma, s, \Psi_{2}^{\nu}\right) \text {. }
\end{aligned}
$$


Здесь $\Psi_{2}^{D}=\Psi_{2}^{D}(s, \mu, \varepsilon)=\Psi_{2}(x, \mu, \varepsilon), x \in \partial \Omega$. Следовательно, функции $w_{k, i}^{(j)}$ должны удовлетворять асимптотикам

$$
w_{k, i}^{(j)}=W_{k, i}+o\left(|\varsigma|^{i}\right), \quad \varsigma \rightarrow \infty .
$$

Задачи для коэффициентов внутреннего разложения получаем, как и ранее, подставляя (2.4) и (2.32) в (1.1), (1.2) и выписывая коэффишиенты при старших степенях $\varepsilon$ и $\eta$ :

$$
\begin{aligned}
\Delta_{\varsigma} w_{k, 1}^{(j)}=- & \frac{\theta_{\varepsilon}^{\prime \prime}}{\left(\theta_{\varepsilon}^{\prime}\right)^{2}}\left(\frac{\partial}{\partial \varsigma_{1}}+2 \varsigma_{2} \frac{\partial^{2}}{\partial \varsigma_{1} \partial \varsigma_{2}}\right) w_{k-1,0}^{(j)} \\
- & \frac{K}{\theta_{\varepsilon}^{\prime}}\left(\frac{\partial}{\partial \varsigma_{2}}-2 \varsigma_{2} \frac{\partial^{2}}{\partial \varsigma_{1}^{2}}\right) w_{k-1,0}^{(j)}-\frac{2}{\theta_{\varepsilon}^{\prime}} \frac{\partial^{2}}{\partial \varsigma_{1} \partial s} w_{k-1,0}^{(j)}, \quad \varsigma_{2}>0, \\
& w_{k, 1}^{(j)}=0, \quad \varsigma \in \gamma_{j}^{1}, \quad \frac{\partial}{\partial \varsigma_{2}} w_{k, 1}^{(j)}=0, \quad \varsigma \in \Gamma_{j}^{1},
\end{aligned}
$$

$k=2,3,4 ;$ а для $w_{1,1}^{(j)}$ и $w_{k, 0}^{(j)}, k=2,3$, получаем ту же краевую задачу $(2.24)$, что и для $w_{1,0}^{(j)}$. Функции $w_{k, 0}^{(j)}, k=2,3$, определим следующим образом:

$$
w_{k, 0}^{(j)}=-\frac{\Psi_{k-1}^{\nu}}{\theta_{\varepsilon}^{\prime}} Y^{(j)}
$$

Имеем $w_{k, 0}^{(j)} \in \mathscr{W}$. Теперь вьпишем асимптотики функций $w_{k, 0}^{(j)}$ (см. $(2.26),(2.38)$ ) и сравним их с асимптотиками (2.36), (2.35). Тогда получим два равенства:

$$
\begin{aligned}
& \frac{\Psi_{1}^{\nu}(s, \mu, \varepsilon)}{\theta_{\varepsilon}^{\prime}(s)} \ln \left(\alpha^{j}(\varepsilon)+\beta^{j}(\varepsilon)\right)=\Psi_{2}^{D}(s, \mu, \varepsilon)+\frac{\Psi_{2}^{\nu}(s, \mu, \varepsilon)}{(A+\mu) \theta_{\varepsilon}^{\prime}(s)}, \\
& \frac{\Psi_{2}^{\nu}(s, \mu, \varepsilon)}{\theta_{\varepsilon}^{\prime}(s)} \ln \left(\alpha^{j}(\varepsilon)+\beta^{j}(\varepsilon)\right)=-\frac{\zeta(3)}{4} l(s, \mu, \varepsilon) .
\end{aligned}
$$

Из первого равенства получим граничное условие для $\Psi_{2}$ :

$$
\left(\frac{\partial}{\partial \nu}+(A+\mu) \theta_{\varepsilon}^{\prime}(s)\right) \Psi_{2}=(A+\mu) \Psi_{1}^{\nu}(s, \mu, \varepsilon) \ln g_{\varepsilon}\left(\theta_{\varepsilon}(s)\right), \quad x \in \partial \Omega,
$$

а из второго определим функцию $l$ :

$$
l(s, \mu, \varepsilon)=-\frac{4}{\zeta(3) \theta_{\varepsilon}^{\prime}(s)} \Psi_{2}^{\nu}(s, \mu, \varepsilon) \ln g_{\varepsilon}\left(\theta_{\varepsilon}(s)\right) .
$$

Краевая задача (2.33), (2.39) однозначно разрешима. Правые части в уравнении (2.33) и граничном условии (2.39) содержат гладкие по $x$ и $s$ функции, а потому $\Psi_{2} \in C^{\infty}(\bar{\Omega})$.

Вернемся к построению внутреннего разложения. Несложно проверить, что функция

$$
Y_{1}^{(j)}(\varsigma, \varepsilon)=\left(\alpha^{j}(\varepsilon)+\beta^{j}(\varepsilon)\right) \operatorname{Re} \sqrt{y^{2}-1}
$$

принадлежит $\mathscr{W}$ и является решением краевой задачи $(2.24)$ с асимптотикой

$$
Y_{1}^{(j)}(\varsigma, \varepsilon)=\varsigma_{1}+O\left(|\varsigma|^{-1}\right), \quad \varsigma \rightarrow \infty .
$$


В силу этой асимптотики и других перечисленных свойств $Y_{1}^{(j)}$ функция $w_{1,1}^{(j)}$ имеет вид

$$
w_{1,1}^{(j)}=-\frac{1}{(A+\mu) \theta_{\varepsilon}^{\prime}}\left(\frac{\Psi_{0}^{\nu}}{\theta_{\varepsilon}^{\prime}}\right)^{\prime} Y_{1}^{(j)}
$$

Прямыми вычислениями несложно проверить, что функция

$$
\widetilde{w}_{2,1}=\frac{\Psi_{0}^{\nu}}{2\left(\theta_{\varepsilon}^{\prime}\right)^{2}} \varsigma_{2}^{2}\left(\frac{\theta_{\varepsilon}^{\prime \prime}}{\theta_{\varepsilon}^{\prime}} \frac{\partial}{\partial \varsigma_{1}}+K \frac{\partial}{\partial \varsigma_{2}}\right) Y^{(j)}+\frac{1}{\theta_{\varepsilon}^{\prime}}\left(\frac{\Psi_{0}^{\nu}}{\theta_{\varepsilon}^{\prime}}\right)^{\prime} \varsigma_{1} Y^{(j)}
$$

принадлежащая $\mathscr{W}$, является решением задачи (2.37) и удовлетворяет следующей асимптотике на бесконечности $(\varsigma \rightarrow \infty)$ :

$\widetilde{w}_{2,1}^{(j)}=\frac{\Psi_{0}^{\nu}}{2\left(\theta_{\varepsilon}^{\prime}\right)^{2}} \frac{\varsigma_{2}^{2}}{|\varsigma|^{2}}\left(\frac{\theta_{\varepsilon}^{\prime \prime}}{\theta_{\varepsilon}^{\prime}} \varsigma_{1}+K \varsigma_{2}\right)+\frac{1}{\theta_{\varepsilon}^{\prime}}\left(\frac{\Psi_{0}^{\nu}}{\theta_{\varepsilon}^{\prime}}\right)^{\prime} \varsigma_{1}\left(\ln |\varsigma|+\ln 2-\ln \left(\alpha^{j}+\beta^{j}\right)\right)+O(1)$.

Для того чтобы получить требуемую функцию $w_{2,1}^{(j)}$, к $\widetilde{w}_{2,1}^{(j)}$ следует добавить гармоническую функцию $Y_{1}^{(j)}$ с подходящим множителем так, чтобы в асимптотике $w_{2,1}^{(j)}$ добиться требуемого коэффициента перед $\varsigma_{1}$. Таким множителем является функция

то есть

$$
\frac{1}{\theta_{\varepsilon}^{\prime}}\left(\frac{\Psi_{0}^{\nu}}{\theta_{\varepsilon}^{\prime}}\right)^{\prime}\left(\ln \left(\alpha^{j}+\beta^{j}\right)-1\right)-\frac{1}{(A+\mu) \theta_{\varepsilon}^{\prime}}\left(\frac{\Psi_{1}^{\nu}}{\theta_{\varepsilon}^{\prime}}\right)^{\prime}
$$

$$
w_{2,1}^{(j)}=\widetilde{w}_{2,1}^{(j)}+\frac{1}{\theta_{\varepsilon}^{\prime}}\left(\left(\frac{\Psi_{0}^{\nu}}{\theta_{\varepsilon}^{\prime}}\right)^{\prime}\left(\ln \left(\alpha^{j}+\beta^{j}\right)-1\right)-\frac{1}{(A+\mu)}\left(\frac{\Psi_{1}^{\nu}}{\theta_{\varepsilon}^{\prime}}\right)^{\prime}\right) Y_{1}^{(j)} .
$$

Аналогично строим $w_{3,1}^{(j)}$ и $w_{4,1}^{(j)}$ :

$$
\begin{aligned}
w_{3,1}^{(j)}= & \frac{\Psi_{1}^{\nu}}{2\left(\theta_{\varepsilon}^{\prime}\right)^{2}} \varsigma_{2}^{2}\left(\frac{\theta_{\varepsilon}^{\prime \prime}}{\theta_{\varepsilon}^{\prime}} \frac{\partial}{\partial \varsigma_{1}}+K \frac{\partial}{\partial \varsigma_{2}}\right) Y^{(j)}+\frac{1}{\theta_{\varepsilon}^{\prime}}\left(\frac{\Psi_{1}^{\nu}}{\theta_{\varepsilon}^{\prime}}\right)^{\prime} \varsigma_{1} Y^{(j)} \\
& +\frac{1}{\theta_{\varepsilon}^{\prime}}\left(\left(\frac{\Psi_{1}^{\nu}}{\theta_{\varepsilon}^{\prime}}\right)^{\prime}\left(\ln \left(\alpha^{j}+\beta^{j}\right)-1\right)-\frac{1}{(A+\mu)}\left(\frac{\Psi_{2}^{\nu}}{\theta_{\varepsilon}^{\prime}}\right)^{\prime}\right) Y_{1}^{(j)} \\
w_{4,1}^{(j)}= & \frac{\Psi_{2}^{\nu}}{2\left(\theta_{\varepsilon}^{\prime}\right)^{2}} \varsigma_{2}^{2}\left(\frac{\theta_{\varepsilon}^{\prime \prime}}{\theta_{\varepsilon}^{\prime}} \frac{\partial}{\partial \varsigma_{1}}+K \frac{\partial}{\partial \varsigma_{2}}\right) Y^{(j)} \\
& +\frac{1}{\theta_{\varepsilon}^{\prime}}\left(\frac{\Psi_{2}^{\nu}}{\theta_{\varepsilon}^{\prime}}\right)^{\prime}\left(\varsigma_{1} Y^{(j)}+\left(\ln \left(\alpha^{j}+\beta^{j}\right)-1\right) Y_{1}^{(j)}\right) .
\end{aligned}
$$

Очевидно, что $w_{k, 1}^{(j)} \in \mathscr{W}$. Используя асимптотики $(2.26)$ и $(2.40)$, непосредственно проверяем, что при $\varsigma \rightarrow \infty$

$$
w_{k, i}^{(j)}=W_{k, i}+O\left(|\varsigma|^{i-1}\right) .
$$

Формальное построение внешнего разложения (2.30), пограничного слоя (2.31) и внутреннего разложения (2.32) закончено.

Следуюшие четыре вспомогательные леммы необходимы для доказательства того, что формально построенные асимптотики собственных элементов являются формальным асимптотическим решением возмущенной задачи.

Пусть $\Omega^{\mathrm{bl}}=\left\{x: 0<\tau<c_{0}\right\}$, где $c_{0}$ - некоторое фиксированное малое число такое, что на множестве $\Omega^{\mathrm{bl}}$ координаты $(s, \tau)$ определены корректно, а функция $P(s, \tau)$ не имеет нулей. Всюду далее через $C$ будем обозначать различные не специфические константы, не зависящие от $\varepsilon$ и $\mu$. 
Лемма 2.2. Пусть $F=F(x, \mu, \varepsilon)$ u $f=f(s, \mu, \varepsilon)$ - бесконечно дифферениируемые по $x$ us функиии, $l_{0}=l_{0}(\mu, \varepsilon)$ - некоторая функция, равномерно ограниченная по в и $\mu$, а норми $\|f\|_{C(\partial \Omega)},\|F\|_{C(\bar{\Omega})} u\|F\|_{C^{k}\left(\bar{\Omega}_{1}\right)}, \quad \Omega_{1} \Subset \Omega-$ произвольная подобласть, $k \in \mathbb{N}$, ограничены равномерно по є и $\mu$. Если краевая задача

$$
\left(\Delta+l_{0}\right) u=F, \quad x \in \Omega, \quad\left(\frac{\partial}{\partial \nu}+(A+\mu) \theta_{\varepsilon}^{\prime}\right) u=f, \quad x \in \partial \Omega,
$$

имеет решение с ограниченной по $\varepsilon$ и $\mu$ нормой в $H^{1}(\Omega)$, то для әтого решения верны равномерные по в и $\mu$ оценки

$$
\begin{aligned}
\|u\|_{C^{1}(\Omega)} & \leqslant C\left(\|f\|_{C^{1}(\partial \Omega)}+1\right), \quad\|u\|_{C^{k}\left(\bar{\Omega}_{1}\right)} \leqslant C \\
\left\|u^{\nu}\right\|_{C(\partial \Omega)} & \leqslant C\left(A+\mu+\|f\|_{C(\partial \Omega)}\right), \\
\left\|u^{\nu}\right\|_{C^{i}(\partial \Omega)} & \leqslant C\left(\|f\|_{C^{i}(\partial \Omega)}+1\right), \quad i=1,2 \\
\left\|u^{\nu}\right\|_{C^{3}(\partial \Omega)} & \leqslant C\left(\|F\|_{C^{1}(\bar{\Omega})}+\|f\|_{C^{3}(\partial \Omega)}+1\right)
\end{aligned}
$$

где $u^{\nu}=u^{\nu}(s, \mu, \varepsilon)=\frac{\partial u}{\partial \nu}(x, \mu, \varepsilon), \quad x \in \partial \Omega, \quad k \in \mathbb{Z}$.

ДокАЗАтЕЛЬСтво. Гладкость функций $f$ и $F$ позволяет утверждать, что решение $u$ задачи (2.42) бесконечно дифференцируемо по $x$. Кроме того, в силу ограниченности нормы $\|u\|_{H^{1}(\Omega)}$ для всякой пары строго внутренних подобластей $\Omega_{1} \Subset \Omega_{2} \Subset \Omega$ выполнено

$$
\|u\|_{H^{k+2}\left(\Omega_{1}\right)} \leqslant C\left(\|F\|_{H^{k}\left(\Omega_{2}\right)}+1\right) \leqslant C, \quad k \in \mathbb{N} .
$$

Последние неравенства и теоремы вложения $C^{k}$ в $H^{k+2}$ позволяют утверждать, что верны оценки

$$
\|u\|_{C^{k}\left(\bar{\Omega}_{1}\right)} \leqslant C
$$

На области $\Omega^{\mathrm{bl}}$ сделаем замену функции $u$ :

$$
v(x, \mu, \varepsilon)=u(x, \mu, \varepsilon) e^{-(A+\mu) \theta_{\varepsilon}^{\prime}(s) \tau}\left(l_{1}-l_{2} \tau^{2}\right),
$$

где $l_{1}$ и $l_{2}-$ некоторые положительные числа. В силу (2.42) функция $v$ есть решение задачи

$$
\begin{gathered}
\left(\Delta_{x}+l_{3} \frac{\partial}{\partial x_{1}}+l_{4} \frac{\partial}{\partial x_{2}}+l_{5}\right) v \equiv L_{1} v=\widetilde{F}, \quad x \in \Omega^{\mathrm{bl}}, \\
v=l_{6}, \quad \tau=c_{0}, \quad \frac{\partial v}{\partial \tau}=-l_{1} f, \quad \tau=0,
\end{gathered}
$$

где $\widetilde{F}=e^{-(A+\mu) \theta_{\varepsilon}^{\prime}(s) \tau}\left(l_{1}-l_{2} \tau^{2}\right) F, l_{i}=l_{i}(x, \mu, \varepsilon), \quad i=3,4,5$, и $l_{6}=l_{6}(s, \mu, \varepsilon)$ гладкие функции пространственных переменных, голоморфные по $\mu$, причем $\left\|l_{6}\right\|_{C\left(\left\{\tau=c_{0}\right\}\right)} \leqslant C$ (см. (2.43)), $\left\|l_{i}\right\|_{C^{1}\left(\bar{\Omega}^{\mathrm{bl}}\right)} \leqslant C$. Функции $l_{i}, i=3,4,5$, легко получить в явном виде, мы не будем приводить здесь этих явных формул, отметим лишь, что за счет подходяшего выбора констант $l_{2}$ и $l_{3}$ и константы $c_{0}$ из определения $\Omega^{\mathrm{bl}}$ всегда можно добиться выполнения неравенств $l_{1}-l_{2} c_{0}^{2}>0, l_{5} \leqslant C<0$ 
при $x \in \bar{\Omega}^{\mathrm{bl}}$. Тогда для оператора $L_{1}$ и всякой функции $V \in C^{2}\left(\bar{\Omega}^{\mathrm{bl}}\right)$ справедливо утверждение: если

$$
L_{1} V<0, \quad x \in \bar{\Omega}^{\mathrm{bl}}, \quad V>0, \quad \tau=c_{0}, \quad \frac{\partial V}{\partial \tau}<0, \quad \tau=0
$$

то $V>0$. Действительно, если допустить обратное, то в точке минимума на $\bar{\Omega}^{\mathrm{bl}}$ функция $V$ отрицательна, $\Delta V \geqslant 0, \nabla_{x} V=0$, т. е. в этой точке $L_{1} V>0$. Ясно, что данная точка минимума лежит строго внутри области $\Omega^{\mathrm{bl}}$; полученное противоречие и доказывает утверждение.

Рассмотрим теперь барьерную “функцию” $\left(l_{7}-l_{8} \tau-l_{9} \tau^{2}\right), l_{7}, l_{8}, l_{9}-$ положительные константы, и применим данное утверждение к функциям $V=\left(l_{7}-l_{8} \tau-\right.$ $\left.l_{9} \tau^{2}\right) \pm v$, каждый раз подходящим образом выбирая константы $l_{i}$. В результате имеем оценку

$$
\begin{aligned}
\|u\|_{C\left(\bar{\Omega}^{\mathrm{bl}}\right)} & \leqslant C\|v\|_{C\left(\bar{\Omega}^{\mathrm{bl}}\right)} \leqslant C\left(\|\widetilde{F}\|_{C\left(\bar{\Omega}^{\mathrm{bl}}\right)}+\|f\|_{C(\partial \Omega)}+\left\|l_{6}\right\|_{C\left(\left\{\tau=c_{0}\right\}\right)}\right) \\
& \leqslant C\left(\|F\|_{C\left(\bar{\Omega}^{\mathrm{bl}}\right)}+\|f\|_{C(\partial \Omega)}+\|u\|_{C\left(\Omega \backslash \Omega^{\mathrm{bl}}\right)}\right) \leqslant C
\end{aligned}
$$

Объединяя последнее неравенство с (2.43), окончательно получаем

$$
\|u\|_{C(\bar{\Omega})} \leqslant C
$$

В книге [36, гл. $3, \S 3$, теорема 3.1] приводится оценка, из которой, производя деление уравнения и краевого условия в (2.42) на достаточно большое фиксированное число и учитывая гладкость $u$, получаем

$$
\|u\|_{C^{2}(\bar{\Omega})} \leqslant C\left(\|F\|_{C(\bar{\Omega})}+\|f\|_{C^{1}(\partial \Omega)}+\|u\|_{C(\bar{\Omega})}\right) .
$$

Из неравенств (2.44) и (2.45) выводим

$$
\|u\|_{C^{2}(\bar{\Omega})} \leqslant C\left(\|f\|_{C^{1}(\partial \Omega)}+1\right)
$$

откуда имеем, в частности, требуемую оценку величины $\|u\|_{C^{1}(\bar{\Omega})}$. В силу граничных условий $u^{\nu}=-(A+\mu) \theta_{\varepsilon}^{\prime} u+f$, а потому, используя $(2.44)$ и (2.46) и учитывая ограниченность $\left\|\theta_{\varepsilon}^{\prime}\right\|_{C^{2}(\partial \Omega)}$, выводим указанные в утверждении леммы оценки величин $\left\|u^{\nu}\right\|_{C^{i}(\partial \Omega)}, i=0,1,2$. Оценим теперь $\left\|u^{\nu}\right\|_{C^{3}(\partial \Omega)}$. При $x \in \Omega^{\mathrm{bl}}$ продифференщируем задачу (2.42) по $s$. Тогда получим, что функция $U=\frac{\partial u}{\partial s}$ есть решение краевой задачи

$$
\begin{gathered}
\left(\Delta_{x}+\frac{\partial}{\partial s}\left(P^{-2}\right)^{\prime} \frac{\partial}{\partial s}+l_{0}\right) U=\frac{\partial F}{\partial s}-\frac{K^{\prime}}{P^{2}} \frac{\partial u}{\partial \tau} \equiv F_{1}, \quad x \in \Omega^{\mathrm{bl}} \\
\left(\frac{\partial}{\partial \nu}+(A+\mu) \theta_{\varepsilon}^{\prime}\right) U=f^{\prime}-(A+\mu) \theta_{\varepsilon}^{\prime \prime} U \equiv f_{1}, \quad x \in \partial \Omega \\
\frac{\partial U}{\partial \tau}=\frac{\partial^{2} u}{\partial s \partial \tau}, \quad \tau=c
\end{gathered}
$$


Для такой задачи, вновь используя результаты [36, гл. $3, \S 3$, теорема 3.1$]$, можно выписать оценку типа (2.45); здесь она будет иметь следующий вид:

$$
\|U\|_{C^{2}\left(\bar{\Omega}^{\mathrm{bl}}\right)} \leqslant C\left(\left\|F_{1}\right\|_{C\left(\bar{\Omega}^{\mathrm{bl}}\right)}+\left\|f_{1}\right\|_{C^{1}(\partial \Omega)}+\left\|\frac{\partial^{2} u}{\partial s \partial \tau}\right\|_{C^{1}\left(\left\{\tau=c_{0}\right\}\right)}+\|U\|_{C\left(\bar{\Omega}^{\mathrm{bl}}\right)}\right) .
$$

Величину $\left\|\frac{\partial^{2} u}{\partial s \partial \tau}\right\|_{C^{1}\left(\left\{\tau=c_{0}\right\}\right)}$ оценим сверху некоторой константой $C$ в силу (2.43); сумму остальных трех слагаемых можно оценить с помошью (2.46):

$$
\left\|F_{1}\right\|_{C\left(\bar{\Omega}^{\mathrm{bl}}\right)}+\left\|f_{1}\right\|_{C^{1}(\partial \Omega)}+\|U\|_{C\left(\bar{\Omega}^{\mathrm{bl}}\right)} \leqslant C\left(\|F\|_{C^{1}\left(\bar{\Omega}^{\mathrm{bl}}\right)}+\|f\|_{C^{2}(\partial \Omega)}+1\right) .
$$

Подставляя теперь полученную оценку в (2.47), приходим к неравенству

$$
\left\|\frac{\partial u}{\partial s}\right\|_{C^{2}\left(\bar{\Omega}^{\mathrm{bl}}\right)} \leqslant C\left(\|F\|_{C^{1}(\bar{\Omega})}+\|f\|_{C^{2}(\partial \Omega)}+1\right)
$$

отсюда и из равенства $u^{\nu}=-(A+\mu) \theta_{\varepsilon}^{\prime} u+f$ и ограниченности $\left\|\theta_{\varepsilon}^{\prime}\right\|_{C^{3}(\partial \Omega)}$ вытекает требуемая оценка $\left\|u^{\nu}\right\|_{C^{3}(\partial \Omega)}$. Лемма доказана.

Лемма 2.3. Функиии $\Psi_{1}$ и $\Lambda_{1}$ представимь в следующем виде:

$$
\Psi_{1}(x, \mu, \varepsilon)=(A+\mu)^{2} \widetilde{\Psi}_{1}(x, \mu, \varepsilon), \quad \Lambda_{1}(\mu, \varepsilon)=(A+\mu)^{2} \widetilde{\Lambda}_{1}(\mu, \varepsilon),
$$

где $\widetilde{\Psi}_{1}$ бесконечно дифферениируема по $x, \widetilde{\Psi}_{1}$ и $\widetilde{\Lambda}_{1}$ голоморфны по $\mu$ для каждого фиксированного значения $\varepsilon$. Верны равномерные по $\varepsilon$ и $\mu$ оценки $(i=1,2,3)$ :

$$
\begin{array}{ccc}
\left|\Lambda_{0}\right| \leqslant C, & \left\|\Psi_{0}\right\|_{H^{1}(\Omega)} \leqslant C, & \left\|\Psi_{0}^{\nu}\right\|_{C^{3}(\partial \Omega)} \leqslant C(A+\mu), \\
\left|\Lambda_{1}\right| \leqslant C(A+\mu)^{2}, & \left\|\Psi_{1}\right\|_{H^{1}(\Omega)} \leqslant C(A+\mu)^{2}, \quad\left\|\Psi_{2}\right\|_{H^{1}(\Omega)} \leqslant C(A+\mu)^{3}, \\
\left\|\Psi_{1}^{\nu}\right\|_{C(\partial \Omega)} \leqslant C(A+\mu)^{2}, & \left\|\Psi_{1}^{\nu}\right\|_{C^{i}(\partial \Omega)} \leqslant C(A+\mu)^{2}\left(\varepsilon^{-i} \delta^{*}(\varepsilon)+1\right), \\
\left\|\Psi_{2}^{\nu}\right\|_{C(\partial \Omega)} \leqslant C(A+\mu)^{3}, & \left\|\Psi_{2}^{\nu}\right\|_{C^{i}(\partial \Omega)} \leqslant C(A+\mu)^{3}\left(\varepsilon^{-i} \delta^{*}(\varepsilon)+1\right) .
\end{array}
$$

ДокАЗАТЕЛЬСТво. Представления (2.48) доказываются весьма просто. В самом деле, для $\Lambda_{1}$ это есть прямое следствие (1.11). Используя представление для $\Lambda_{1}$ и наличие множителя $(A+\mu)^{2}$ в граничном условии $(2.28)$, приходим к требуемому представлению для $\Psi_{1}$.

Оценки для $\Lambda_{0}$ и $\Psi_{0}$ из $(2.49)$ доказываются элементарно. Ограниченность $\Lambda_{0}$ следует из сходимости $\Lambda_{0} \rightarrow \lambda_{0}$. Поскольку $\left\|\Psi_{0}\right\|_{L_{2}(\Omega)}=1$, то, умножая уравнение (1.7) на $\Psi_{0}$ и интегрируя один раз по частям, в силу ограниченности $\Lambda_{0}$ и $\left\|\theta_{\varepsilon}^{\prime}\right\|_{C(\partial \Omega)}$ получаем требуемую оценку нормы $\left\|\Psi_{0}\right\|_{H^{1}(\Omega)}$. Теперь, применяя лемму 2.2 к задаче для функции $\Psi_{0}$, получаем оценку $\left\|\Psi_{0}^{\nu}\right\|_{C^{3}(\partial \Omega)}$, и, кроме того, $\left\|\Psi_{0}\right\|_{C^{1}(\bar{\Omega})} \leqslant C$, $\left\|\Psi_{0}\right\|_{C^{k}\left(\bar{\Omega}_{1}\right)} \leqslant C$ для всякой подобласти $\Omega_{1} \Subset \Omega$.

Оценка для $\Lambda_{1}$ вытекает из доказанных оценок для $\Psi_{0}$, ограниченности функций $\theta_{\varepsilon}^{\prime}$ и $g_{\varepsilon}\left(\theta_{\varepsilon}\right)$ и формулы (1.11). 
Неравенства для $\Psi_{1}$ и $\Psi_{2}$ из (2.49) также докажем на основе леммы 2.2. Поскольку $\Psi_{1}$ ортогональна $\Psi_{0}$ в $L_{2}(\Omega)$, а величины $\Lambda_{0}$ и $\Lambda_{1}$ ограничены, то верна равномерная оценка

$$
\left\|\Psi_{1}\right\|_{H^{1}(\Omega)} \leqslant C(A+\mu)^{2}\left(\left\|\Psi_{0}\right\|_{L_{2}(\Omega)}+\left\|\Psi_{0}^{D} \theta_{\varepsilon}^{\prime} \ln g_{\varepsilon}\left(\theta_{\varepsilon}\right)\right\|_{H^{1}(\Omega)}\right) \leqslant C(A+\mu)^{2} .
$$

Правые части уравнения (2.8) и граничного условия (2.28) удовлетворяют всем условиям леммы 2.2. Кроме того, заметим, что оценки производных граничного условия (2.28) фактически сводятся к оценкам производных (ограниченной) функции $g_{\varepsilon}\left(\theta_{\varepsilon}(s)\right)$, так как производные $\theta_{\varepsilon}^{\prime}$ оцениваются в силу условия $(\mathrm{C} 0)$, а производные $\Psi_{0}^{D}$ оцениваются с помощью уже доказанных оценок для $\Psi_{0}^{\nu}$ и равенства $\Psi_{0}^{\nu}=(A+\mu) \theta_{\varepsilon}^{\prime} \Psi_{0}^{D}$. Производные $g_{\varepsilon}\left(\theta_{\varepsilon}\right)$, очевидно, оцениваются следуюшим образом:

$$
\left\|g_{\varepsilon}\left(\theta_{\varepsilon}(s)\right)\right\|_{C^{i}(\partial \Omega)} \leqslant C\left(\varepsilon^{-i} \delta^{*}(\varepsilon)+1\right), \quad i=1,2,3 .
$$

Используя этот очевидный факт и применяя лемму 2.2 к задаче для $\widetilde{\Psi}_{1}$, приходим к оценкам для $\Psi_{1}$ из (2.49). Кроме того, в силу леммы 2.2 выполнены неравенства

$$
\left\|\Psi_{1}\right\|_{C^{1}(\bar{\Omega})} \leqslant C(A+\mu)^{2}\left(\varepsilon^{-1} \delta^{*}(\varepsilon)+1\right), \quad\left\|\Psi_{1}\right\|_{C^{k}\left(\bar{\Omega}_{1}\right)} \leqslant C
$$

для всех $k \in \mathbb{Z}_{+}$и всех $\Omega_{1} \Subset \Omega$. Из очевидного неравенства

$$
\left\|\Psi_{2}\right\|_{H^{1}(\Omega)} \leqslant C\left(\left|\Lambda_{1}\right|\left\|\Psi_{1}\right\|_{L_{2}(\Omega)}+(A+\mu)\left\|\Psi_{1}^{\nu}\right\|_{L_{2}(\partial \Omega)}\right)
$$

и уже доказанных оценок для $\Psi_{1}$ и $\Lambda_{1}$ выводим требуемую оценку для нормы $\left\|\Psi_{2}\right\|_{H^{1}(\Omega)}$. Представляя $\Psi_{2}$ в виде $\Psi_{2}=(A+\mu)^{3} \widetilde{\Psi}_{2}$ и применяя к $\widetilde{\Psi}_{2}$ лемму 2.2 , получаем и остальные оценки для $\Psi_{2}$ из (2.49). Лемма доказана.

Обозначим $\widehat{\lambda}_{\varepsilon}=\Lambda_{0}(\mu, \varepsilon)+\varepsilon \Lambda_{1}(\mu, \varepsilon), \Pi^{(j)}=\left\{\xi:\left|\xi_{1}-\pi j\right|<\pi / 2, \xi_{2}>0\right\}$, $\Pi_{\eta}^{(j)}=\Pi^{(j)} \cap\left\{\xi: 4\left|\xi-\xi^{(j)}\right|>\eta^{1 / 4}\right\}, \Omega_{\eta}^{\mathrm{bl}}=\Omega^{\mathrm{bl}} \cap\left\{x: \xi \in \Pi_{\eta}^{(j)}, j=0, \ldots, N-1\right\}$.

ЛЕмма 2.4. Для пограничного слоя (2.31) при $\varepsilon \rightarrow 0$ справедливы следующие равномерные оченки:

$$
\begin{gathered}
\left\|\psi_{\varepsilon}^{\mathrm{bl}}\right\|_{L_{2}\left(\Omega^{\mathrm{bl}}\right)} \leqslant C \varepsilon^{3 / 2}(A+\mu), \\
\left\|\psi_{\varepsilon}^{\mathrm{bl}}-\varepsilon v_{1}-\varepsilon^{2}\left(v_{2}-\tilde{v}_{2}\right)\right\|_{H^{1}\left(\Omega_{\eta}^{\mathrm{bl}}\right)} \leqslant C \varepsilon^{3 / 2}(A+\mu)^{2}, \\
\left\|\left(\Delta_{x}+\widehat{\lambda}_{\varepsilon}\right) \psi_{\varepsilon}^{\mathrm{bl}}\right\|_{L_{2}\left(\Omega^{\mathrm{bl}}\right)} \leqslant C\left(\varepsilon^{3 / 2}(A+\mu)+\varepsilon^{1 / 2} \delta^{*}(\varepsilon)(A+\mu)^{2}\right) .
\end{gathered}
$$

ДокАЗАТЕЛЬСТво. Всюду в доказательстве леммы, не оговаривая это дополнительно, будем опираться на тот очевидный факт, что

$$
\xi_{2}^{i+k} \frac{\partial^{i} X}{\partial \xi_{2}^{i}}, \quad \xi_{2}^{i+k} \frac{\partial^{i} X}{\partial \xi_{1} \partial \xi_{2}^{i-1}} \in L_{2}\left(\Pi^{(j)}\right) \cap \mathscr{V}_{0}, \quad i, k \in \mathbb{Z}, \quad i, k \geqslant 0 .
$$

Верна оценка

$$
\left\|\psi_{\varepsilon}^{\mathrm{bl}}\right\|_{L_{2}\left(\Omega^{\mathrm{bl}}\right)} \leqslant \varepsilon\left(\sum_{j=0}^{N-1}\left\|\psi_{\varepsilon}^{\mathrm{bl}}\right\|_{L_{2}\left(\Pi^{(j)}\right)}^{2}\right)^{1 / 2}
$$


Нормы $\left\|\psi_{\varepsilon}^{\mathrm{bl}}\right\|_{L_{2}\left(\Pi^{(j)}\right)}$ оценим, используя явный вид функций $v_{i}$ и оценки из леммы 2.3:

$$
\left\|\psi_{\varepsilon}^{\mathrm{bl}}\right\|_{L_{2}\left(\Pi^{(j)}\right)} \leqslant C \varepsilon(A+\mu) .
$$

Из последних двух оценок и равенства $N=2 \varepsilon^{-1}$ следует первое неравенство из утверждения леммы. Второе неравенство из утверждения леммы доказывается аналогично, на основе явного вида функций $v_{i}$ и леммы 2.3. Для краткости обозначим $F_{\varepsilon}=\left(\Delta_{x}+\widehat{\lambda}_{\varepsilon}\right) \psi_{\varepsilon}^{\mathrm{bl}}$. Используя лвный вид функций $v_{i}$, вычислим

$$
\begin{aligned}
P^{3} F_{\varepsilon}= & \varepsilon \sum_{i=1}^{3} \xi_{2}^{i}\left(T_{0, i} \frac{\partial^{i} X}{\partial \xi_{2}^{i}}+T_{1, i-1} \frac{\partial^{i} X}{\partial \xi_{1} \partial \xi_{2}^{i-1}}\right)+\varepsilon T_{0,0} X \\
& +\varepsilon^{2} T_{1,-1} \int_{\xi_{2}}^{+\infty} t \frac{\partial}{\partial \xi_{1}} X\left(\xi_{1}, t\right) d t+\varepsilon T_{0,-1} \int_{\xi_{2}}^{+\infty} t X\left(\xi_{1}, t\right) d t .
\end{aligned}
$$

Здесь $T_{i, k}=T_{i, k}\left(\xi_{2}, s, \varepsilon, \mu\right)$ - полиномы по $\xi_{2}$ с коэффициентами, зависящими от остальных переменных, причем эти коэффициенты в силу леммы 2.3 оцениваются сверху величиной $C\left((A+\mu)+\varepsilon^{-1} \delta^{*}(\varepsilon)(A+\mu)^{2}\right)$, где $C$ не зависит от $\varepsilon, \mu, s$. Используя указанные оценки коэффициентов полиномов $T_{i, k}$ и вид функции $P^{3} F_{\varepsilon}$, убеждаемся, что

$$
\left\|P^{3} F_{\varepsilon}\right\|_{L_{2}\left(\Pi^{(j)}\right)} \leqslant C\left(\varepsilon(A+\mu)+\delta^{*}(\varepsilon)(A+\mu)^{2}\right),
$$

где $C$ не зависит от $\varepsilon, \mu, s$ и $j$. Поскольку при $x \in \bar{\Omega}^{\text {bl }}$ функция $P$ не обрашается в нуль, то

$$
\left\|F_{\varepsilon}\right\|_{L_{2}\left(\Omega^{\mathrm{bl}}\right)} \leqslant C\left\|P^{3} F_{\varepsilon}\right\|_{L_{2}\left(\Omega^{\mathrm{bl}}\right)} \leqslant C \varepsilon\left(\sum_{j=0}^{N-1}\left\|P^{3} F_{\varepsilon}\right\|_{L_{2}\left(\Pi^{(j)}\right)}^{2}\right)^{1 / 2},
$$

откуда вместе с уже полученными оценками норм $\left\|P^{3} F_{\varepsilon}\right\|_{L_{2}\left(\Pi^{(j)}\right)}$ получаем третье неравенство утверждения леммы. Лемма доказана.

Обозначим $\Omega_{j}^{\text {in }}=\left\{x: 4 \eta^{3 / 4}\left|\varsigma^{j}\right|<3\right\}, \Omega_{j}^{\text {mat }}=\left\{x: 1<4\left|\varsigma^{j}\right| \eta^{3 / 4}<3, j=\right.$ $0, \ldots, N-1\}$.

Аналогично лемме 2.4 доказывается справедливость следуюшего утверждения.

ЛЕмма 2.5. Для внутреннего разложсения (2.32) при $\varepsilon \rightarrow 0$ справедливы следующие равномерные по $\varepsilon, \mu$ и $\eta$ оченки:

$$
\begin{gathered}
\left\|\psi_{\varepsilon}^{\text {in }, j}\right\|_{L_{2}\left(\Omega_{j}^{\text {in }}\right)} \leqslant C \eta^{1 / 5}, \quad\left\|\left(\Delta_{x}+\widehat{\lambda}_{\varepsilon}\right) \psi_{\varepsilon}^{\text {in }, j}\right\|_{L_{2}\left(\Omega_{j}^{\text {in }}\right)} \leqslant C \eta^{1 / 5}, \\
\left\|\psi_{\varepsilon}^{\text {in }, j}-\varepsilon w_{1,0}^{(j)}-\varepsilon^{2} w_{2,0}^{(j)}\right\|_{H^{1}\left(\Omega_{j}^{\text {in }}\right)} \leqslant C \varepsilon^{2}(A+\mu)^{3 / 2} .
\end{gathered}
$$

Пусть

$$
\widehat{\psi}_{\varepsilon}(x)=\left(\psi_{\varepsilon}^{\mathrm{ex}}(x, \mu)+\chi\left(\tau / c_{0}\right) \psi_{\varepsilon}^{\mathrm{bl}}(\xi, s, \mu)\right) \chi_{\varepsilon}(x)+\sum_{j=0}^{N-1} \chi\left(\left|\varsigma^{j}\right| \eta^{3 / 4}\right) \psi_{\varepsilon}^{\mathrm{in}, j}\left(\varsigma^{j}, s, \mu\right),
$$

где $\psi_{\varepsilon}^{\mathrm{ex}}, \psi_{\varepsilon}^{\mathrm{bl}}$ и $\psi_{\varepsilon}^{\mathrm{in}, j}$ из $(2.30)-(2.32)$,

$$
\chi_{\varepsilon}(x)=1-\sum_{j=0}^{N-1} \chi\left(\left|\varsigma^{j}\right| \eta^{3 / 4}\right) .
$$

В следующем утверждении докажем, что формально построенные асимптотики $\widehat{\lambda}_{\varepsilon}$ и $\widehat{\psi}_{\varepsilon}$ являются формальным асимптотическим решением возмущенной задачи. 
ЛЕмма 2.6. Функиии $\widehat{\psi}_{\varepsilon} \in C^{\infty}\left(\Omega \cup \gamma_{\varepsilon} \cup \Gamma_{\varepsilon}\right) \cap H^{1}(\Omega)$ и $\widehat{\lambda}_{\varepsilon}$ удовлетворяют краевой задаче

$$
-\Delta u_{\varepsilon}=\lambda u_{\varepsilon}+f, \quad x \in \Omega, \quad u_{\varepsilon}=0, \quad x \in \gamma_{\varepsilon}, \quad \frac{\partial u_{\varepsilon}}{\partial \nu}=0, \quad x \in \Gamma_{\varepsilon},
$$

при $u_{\varepsilon}=\widehat{\psi}_{\varepsilon}, \quad \lambda=\widehat{\lambda}_{\varepsilon} u f=f_{\varepsilon}$, әде для $f_{\varepsilon}$ верна равномерная оценка:

$$
\left\|f_{\varepsilon}\right\|_{L_{2}(\Omega)} \leqslant C\left(\varepsilon^{3 / 2}(A+\mu(\varepsilon))+\varepsilon^{1 / 2} \delta^{*}(\varepsilon)(A+\mu(\varepsilon))^{2}\right) .
$$

Функция $\widehat{\lambda}_{\varepsilon}$ сходится $к \lambda_{0}$ при $\varepsilon \rightarrow 0$, а для функиии $\widehat{\psi}_{\varepsilon}$ справедлива оценка $\left\|\widehat{\psi}_{\varepsilon}-\Psi_{0}\right\|_{L_{2}(\Omega)}=o(1)$.

ДокАЗАТЕльСтво. Утверждение о гладкости функции $\widehat{\psi}_{\varepsilon}$ очевидно. Сходимость $\widehat{\lambda}_{\varepsilon}$ к $\lambda_{0}$ следует из лемм 1.1 и 2.3 . Оценка $\left\|\widehat{\psi}_{\varepsilon}-\Psi_{0}\right\|_{L_{2}(\Omega)}=o(1)$ является прямым следствием лемм 2.3-2.5. Проверим граничные условия из (2.50). Равенство функции $\widehat{\psi}_{\varepsilon}$ на $\gamma_{\varepsilon}$ вытекает из равенства нулю $\chi_{\varepsilon}$ на $\gamma_{\varepsilon}$ и равенства нулю $\psi_{\varepsilon}^{\text {in, }}, j$ на $\gamma_{j}^{1}$. Нетрудно проверить, что при $x \in \Gamma_{\varepsilon}$

$$
\begin{aligned}
\frac{\partial \widehat{\psi}_{\varepsilon}}{\partial \nu} & =\left(\frac{\partial \psi_{\varepsilon}^{\mathrm{ex}}}{\partial \nu}-\left.\frac{\theta_{\varepsilon}^{\prime}}{\varepsilon} \frac{\partial \psi_{\varepsilon}^{\mathrm{b} 1}}{\partial \xi_{2}}\right|_{\xi \in \Gamma^{0}}\right) \chi_{\varepsilon}(x)-\left.\frac{\theta_{\varepsilon}^{\prime}}{\varepsilon \eta} \sum_{j=0}^{N-1} \chi\left(\left|\varsigma^{j}\right| \eta^{3 / 4}\right) \frac{\partial \psi_{\varepsilon}^{\mathrm{in}, j}}{\partial \varsigma_{2}}\right|_{\varsigma \in \Gamma_{j}^{1}} \\
& =\chi_{\varepsilon}(x) \sum_{i=0}^{2} \varepsilon^{i}\left(\Psi_{i}^{\nu}-\left.\theta_{\varepsilon}^{\prime} \frac{\partial v_{i+1}}{\partial \xi_{2}}\right|_{\xi \in \Gamma^{0}}\right)=0 .
\end{aligned}
$$

Оценим функцию $f_{\varepsilon}$. Она, очевидно, имеет следуюший вид:

$$
\begin{aligned}
f_{\varepsilon} & =-\left(\Delta_{x}+\widehat{\lambda}_{\varepsilon}\right) \widehat{\psi}_{\varepsilon}=-\sum_{i=1}^{5} f_{\varepsilon}^{(i)}, \\
f_{\varepsilon}^{(1)} & =\chi_{\varepsilon}\left(\Delta_{x}+\widehat{\lambda}_{\varepsilon}\right) \psi_{\varepsilon}^{\mathrm{ex}}, \\
f_{\varepsilon}^{(2)} & =\chi_{\varepsilon} \chi\left(\tau / c_{0}\right)\left(\Delta_{x}+\widehat{\lambda}_{\varepsilon}\right) \psi_{\varepsilon}^{\mathrm{bl}}, \\
f_{\varepsilon}^{(3)} & =\chi_{\varepsilon}\left(2\left(\nabla_{x} \psi_{\varepsilon}^{\mathrm{bl}}, \nabla_{x} \chi\left(\tau / c_{0}\right)\right)+\psi_{\varepsilon}^{\mathrm{bl}} \Delta_{x} \chi\left(\tau / c_{0}\right)\right), \\
f_{\varepsilon}^{(4)}= & \sum_{j=0}^{N-1} \chi\left(\left|\varsigma^{j}\right| \eta^{3 / 4}\right)\left(\Delta_{x}+\widehat{\lambda}_{\varepsilon}\right) \psi_{\varepsilon}^{\mathrm{in}, j}, \\
f_{\varepsilon}^{(5)}= & \sum_{j=0}^{N-1}\left(2\left(\nabla_{x} \psi_{\varepsilon}^{\mathrm{mat}, j}, \nabla_{x} \chi\left(\left|\varsigma^{j}\right| \eta^{3 / 4}\right)\right)+\psi_{\varepsilon}^{\mathrm{mat}, j} \Delta_{x} \chi\left(\left|\varsigma^{j}\right| \eta^{3 / 4}\right)\right), \\
& \psi_{\varepsilon}^{\mathrm{mat}, j}=\psi_{\varepsilon}^{\mathrm{in}, j}-\psi_{\varepsilon}^{\mathrm{ex}}-\psi_{\varepsilon}^{\mathrm{bl}} .
\end{aligned}
$$

Используя уравнения (1.7), (2.8) и (2.33), нетрудно убедиться в том, что

$$
\left(\Delta_{x}+\widehat{\lambda}_{\varepsilon}\right) \psi_{\varepsilon}^{\mathrm{ex}}=\varepsilon^{2}\left(\Lambda_{0}+1+\varepsilon \Lambda_{1}\right) \Psi_{2},
$$

а потому в силу леммы 2.3 имеем

$$
\left\|f_{\varepsilon}^{(1)}\right\|_{L_{2}(\Omega)} \leqslant C \varepsilon^{2}(A+\mu)^{3} .
$$


Функция $f_{\varepsilon}^{(2)}$ оценивается с помощью леммы 2.4:

$$
\left\|f_{\varepsilon}^{(2)}\right\|_{L_{2}(\Omega)} \leqslant\left\|f_{\varepsilon}^{(2)}\right\|_{L_{2}\left(\Omega^{\mathrm{bl}}\right)} \leqslant C\left(\varepsilon^{3 / 2}(A+\mu)+\varepsilon^{1 / 2} \delta^{*}(\varepsilon)(A+\mu)^{2}\right) .
$$

Функции $v_{i}$ и, следовательно, $\psi_{\varepsilon}^{\mathrm{bl}}$ экспоненциально убывают, а интегрирование $f_{\varepsilon}^{(3)}$ по $\Omega$ в силу определения $\chi$ фактически сводится к интегрированию по области $\left\{x: \frac{c_{0} \theta_{\varepsilon}^{\prime}}{4 \varepsilon} \leqslant \xi_{2} \leqslant \frac{3 c_{0} \theta_{\varepsilon}^{\prime}}{4 \varepsilon}\right\}$, а потому

$$
\left\|f_{\varepsilon}^{(3)}\right\|_{L_{2}(\Omega)} \leqslant C(A+\mu) e^{-1 / \varepsilon^{c_{5}}},
$$

где $c_{5}>0$ - некоторое фиксированное число. Далее, функцию $f_{\varepsilon}^{(4)}$ оцениваем на основе леммы 2.5:

$$
\left\|f_{\varepsilon}^{(4)}\right\|_{L_{2}(\Omega)} \leqslant C \sum_{j=0}^{N-1}\left\|\left(\Delta_{x}+\widehat{\lambda}_{\varepsilon}\right) \psi_{\varepsilon}^{\mathrm{in}, j}\right\|_{L_{2}\left(\Omega_{j}^{\mathrm{in}}\right)} \leqslant C \eta^{1 / 6} .
$$

В силу проведенного согласования (см. (2.34), (2.41)) функция $\psi_{\varepsilon}^{\text {mat }, j}$ при $\eta^{-3 / 4} \leqslant$ $4\left|\varsigma^{j}\right| \leqslant 3 \eta^{-3 / 4}$ имеет следующую дифференцируемую асимптотику:

$$
\psi_{\varepsilon}^{\text {mat }, j}=O\left(\eta^{2}|\varsigma|^{2}|\ln | \varsigma||+\varepsilon|\varsigma|^{-1}+\varepsilon \eta\right),
$$

используя которую, оцениваем $f_{\varepsilon}^{(5)}$ :

$$
\left\|f_{\varepsilon}^{(5)}\right\|_{L_{2}(\Omega)} \leqslant C \sum_{j=0}^{N-1}\left\|f_{\varepsilon}^{(5)}\right\|_{L_{2}\left(\Omega_{j}^{\mathrm{mat}}\right)} \leqslant C \eta^{1 / 5} .
$$

Из полученных оценок величин $f_{\varepsilon}^{(i)}$ следует утверждение леммы. Лемма доказана.

Переходим к обоснованию асимптотик. Аналогично [17], [19], [28], [29], [37] можно показать, что при $\lambda$, близких к $p$-кратному собственному значению $\lambda_{0}$ предельной задачи, для решения задачи (2.50) при $f \in L_{2}(\Omega)$ справедливо представление

$$
u_{\varepsilon}=\sum_{k=q}^{q+p-1} \frac{\psi_{\varepsilon}^{k}}{\lambda_{\varepsilon}^{k}-\lambda} \int_{\Omega} f \psi_{\varepsilon}^{k} d x+\tilde{u}_{\varepsilon}
$$

где $\lambda_{\varepsilon}^{k}, k=q, \ldots, q+p-1,-$ собственные значения возмущенной задачи, сходящиеся к $\lambda_{0}, \psi_{\varepsilon}^{k}$, напомним, соответствуюшие ортонормированные в $L_{2}(\Omega)$ собственные функции, $\tilde{u}_{\varepsilon}$ - голоморфная по $\lambda$ в норме $H^{1}(\Omega)$ функция, ортогональная всем $\psi_{\varepsilon}^{k}$, $k=q, \ldots, q+p-1$, в $L_{2}(\Omega) ;$ для функции $\tilde{u}_{\varepsilon}$ выполнена равномерная по $\varepsilon, \mu, \lambda$ и $f$ оценка

$$
\left\|\tilde{u}_{\varepsilon}\right\|_{H^{1}(\Omega)} \leqslant C\|f\|_{L_{2}(\Omega)} .
$$

В рассматриваемом случае $\lambda_{0}-$ простое собственное значение. Положим $u_{\varepsilon}=\widehat{\psi}_{\varepsilon}$, $\lambda=\widehat{\lambda}_{\varepsilon}$ и $f=f_{\varepsilon}$. Тогда из леммы 2.6 и соотношений $(2.53),(2.54)$ получаем

$$
\begin{gathered}
\widehat{\psi}_{\varepsilon}=\frac{\psi_{\varepsilon}}{\lambda_{\varepsilon}-\widehat{\lambda}_{\varepsilon}} \int_{\Omega} f_{\varepsilon} \psi_{\varepsilon} d x+\tilde{u}_{\varepsilon}, \\
\left\|\tilde{u}_{\varepsilon}\right\|_{H^{1}(\Omega)} \leqslant C\left(\varepsilon^{3 / 2}(A+\mu)+\varepsilon^{1 / 2} \delta^{*}(\varepsilon)(A+\mu)^{2}\right) .
\end{gathered}
$$


Поскольку $\left\|\widehat{\psi}_{\varepsilon}-\tilde{u}_{\varepsilon}\right\|_{L_{2}(\Omega)}=\left\|\Psi_{0}\right\|_{L_{2}(\Omega)}(1+o(1))=1+o(1)$ (см. лемму 2.6), то в силу (2.55) имеем

$$
C \leqslant \frac{\left\|f_{\varepsilon}\right\|_{L_{2}(\Omega)}}{\left|\lambda_{\varepsilon}-\widehat{\lambda}_{\varepsilon}\right|} \Longrightarrow\left|\lambda_{\varepsilon}-\widehat{\lambda}_{\varepsilon}\right| \leqslant C\left(\varepsilon^{3 / 2}(A+\mu)+\varepsilon^{1 / 2} \delta^{*}(\varepsilon)(A+\mu)^{2}\right) .
$$

В силу следствия 2.1 можно заменить в последней оценке функцию $\delta^{*}(\varepsilon)$ на $o\left(\varepsilon^{1 / 2}(A+\mu)^{-1}\right)$, т. е. асимптотика (1.10) верна.

Случай $p$-кратного собственного значения $\lambda_{0}=\lambda_{0}^{q}=\cdots=\lambda_{0}^{q+p-1}$ в целом доказывается аналогично. При построении кратность $\lambda_{0}$ проявляется в том, что по той же схеме мы строим несколько асимптотик, соответствующих собственным значениям $\lambda_{\varepsilon}^{k}$, сходяшимся к $\lambda_{0}$. Кроме того, в построении кратность проявляется при решении задачи $(1.7),(1.8)$, которая будет иметь несколько собственных значений $\Lambda_{0}^{k}$, сходящихся к $\Lambda_{0}$, и, разумеется, несколько собственных функций $\Psi_{0}^{k}$. Собственные значения $\Lambda_{0}^{k}$ удовлетворяют лемме 1.1 , соответствуюшие собственные функции $\Psi_{0}^{k}$ также следует выбрать согласно лемме 1.1. В частности, ортогональность $\Psi_{0}^{k}$ в $L_{2}(\partial \Omega)$ с весом $\theta_{\varepsilon}^{\prime}$ является условием разрешимости задач для функций $\Psi_{1}^{k}$, которые мы вновь выбираем ортогональными $\Psi_{0}^{k}$. Все остальные рассуждения формального построения, в том числе утверждения лемм $2.3-2.5$, остаются в силе. Таким образом, в результате формального построения имеем функции $\widehat{\lambda}_{\varepsilon}^{k}$ и $\widehat{\psi}_{\varepsilon}^{k}, k=q, \ldots, p+q-1$, которые определяются так же, как $\widehat{\lambda}_{\varepsilon}$ и $\widehat{\psi}_{\varepsilon}$, с заменой $\Lambda_{0}$ на $\Lambda_{0}^{k}$ и $\Psi_{0}$ на $\Psi_{0}^{k}$. Для $\widehat{\lambda}_{\varepsilon}^{k}$ и $\widehat{\psi}_{\varepsilon}^{k}$ выполнена лемма 2.6. Через $f_{\varepsilon}^{k}$ обозначим правые части уравнений из (2.50) с $u_{\varepsilon}=\widehat{\psi}_{\varepsilon}^{k}, \lambda=\widehat{\lambda}_{\varepsilon}^{k}$.

Применим представление $(2.53)$ к функциям $\widehat{\psi}_{\varepsilon}^{k}$ :

$$
\begin{gathered}
\widehat{\psi}_{\varepsilon}^{k}=\sum_{i=q}^{q+p-1} h_{k i}^{\varepsilon} \psi_{\varepsilon}^{i}+\tilde{u}_{\varepsilon}^{k}, \quad h_{k i}^{\varepsilon}=\frac{1}{\lambda_{\varepsilon}^{i}-\widehat{\lambda}_{\varepsilon}^{k}} \int_{\Omega} f_{\varepsilon}^{k} \psi_{\varepsilon}^{i} d x, \\
\left\|\tilde{u}_{\varepsilon}^{k}\right\|_{H^{1}(\Omega)} \leqslant C\left(\varepsilon^{3 / 2}(A+\mu)+\varepsilon^{1 / 2} \delta^{*}(\varepsilon)(A+\mu)^{2}\right) .
\end{gathered}
$$

Последняя оценка для $\tilde{u}_{\varepsilon}^{k}$ вытекает из (2.54) и леммы 2.6. В силу (2.56) и ортогональности $\tilde{u}_{\varepsilon}^{k}$ функциям $\psi_{\varepsilon}^{k}$ получаем соотношения

$$
h_{k i}^{\varepsilon}=\left(\widehat{\psi}_{\varepsilon}^{k}, \psi_{\varepsilon}^{i}\right)_{L_{2}(\Omega)},
$$

откуда следует ограниченность величин $h_{k i}^{\varepsilon}$. Докажем теперь асимптотики (1.10) для собственных значений $\lambda_{\varepsilon}^{k}, k=q, \ldots, q+p-1$. Допустим противное, а именно пусть сушествует подпоследовательность $\varepsilon_{m}$, на которой для некоторых собственных значений $\lambda_{\varepsilon}^{k}, k=q, \ldots, q+p-1$, асимптотики (1.10) неверны и для $k=q, \ldots, q+p-1, i \in I \neq \varnothing$ выполнено

$$
\left|\lambda_{\varepsilon_{m}}^{i}-\widehat{\lambda}_{\varepsilon_{m}}^{k}\right| \geqslant m\left(\varepsilon_{m}^{3 / 2}(A+\mu)+\varepsilon_{m}^{1 / 2} \delta^{*}\left(\varepsilon_{m}\right)(A+\mu)^{2}\right),
$$

где $I \subseteq\{q, \ldots, q+p-1\}$ - подмножество индексов собственных значений, которые не удовлетворяют асимптотикам (1.10). В силу оценок для функций $f_{\varepsilon}^{k}$, формул для $h_{k i}^{\varepsilon}$ из $(2.56)$ и неравенств $(2.58)$ получаем

$$
h_{k i}^{\varepsilon_{m}} \underset{m \rightarrow \infty}{\longrightarrow} 0, \quad k=q, \ldots, q+p-1, \quad i \in I .
$$


Учитывая теперь ограниченность $h_{k i}^{\varepsilon_{m}}$ и выделяя при необходимости из $\varepsilon_{m}$ подпоследовательность, считаем, что $h_{k i}^{\varepsilon_{m}} \rightarrow h_{k i}^{0}$, причем в силу (2.59) верны равенства $h_{k i}^{0}=0$ при $k=q, \ldots, q+p-1, k \in I$. Из чисел $h_{k i}^{\varepsilon_{m}}$ составим $p$ векторов $h_{k}^{\varepsilon_{m}}$ по следуюшему правилу: в качестве компонент вектора $h_{k}^{\varepsilon_{m}}$ берем последовательно числа $h_{k i}^{\varepsilon_{m}}$, причем индекс $i$ меняется в пределах от $q$ до $q+p-1$ и не принимает значений из множества $I$. Аналогичным образом из чисел $h_{k i}^{0} \operatorname{cocтавим~} p$ векторов $h_{k}^{0}$. Размерности полученных векторов, очевидно, равны $p-|I|<p$. Теперь умножим скалярно в $L_{2}(\Omega)$ представления $(2.56)$ для $\widehat{\psi}_{\varepsilon}^{k}$ друг на друга при всех значениях $k$ и учтем равенства $\left\|\widehat{\psi}_{\varepsilon}^{k}-\Psi_{0}^{k}\right\|_{L_{2}(\Omega)}=o(1)$, оценки для $\tilde{u}_{\varepsilon}^{k}$ и ортонормированность функций $\Psi_{0}^{k}$ и $\psi_{\varepsilon}^{k}$. Тогда получим

$$
\left(h_{k}^{0}, h_{j}^{0}\right)_{L_{2}(\Omega)}=\lim _{m \rightarrow \infty}\left(h_{k}^{\varepsilon_{m}}, h_{j}^{\varepsilon_{m}^{m}}\right)_{L_{2}(\Omega)}=\delta_{k j}, \quad k, j=q, \ldots, q+p-1
$$

где $\delta_{k j}-$ символ Кронекера, т.е. $h_{k}^{0}$ образуют систему $p$ ортонормированных $(p-|I|)$-мерных векторов. Из полученного противоречия следуют оценки

$$
\left|\lambda_{\varepsilon}^{k}-\widehat{\lambda}_{\varepsilon}^{k}\right| \leqslant C\left(\varepsilon^{3 / 2}(A+\mu)+\varepsilon^{1 / 2} \delta^{*}(\varepsilon)(A+\mu)^{2}\right),
$$

что с учетом равенства (1.9), леммы 2.1 и следствия 2.1 приводит к асимптотикам (1.10) и в случае кратного собственного значения $\lambda_{0}$. Теорема 1.4 полностью доказана.

Выясним теперь асимптотическое поведение собственных функций возмущенной задачи в условиях теоремы 1.4. При выполнении условий (C0), (C1) и равенства (1.5) при $A \geqslant 0$ аналогично работам [2], [6], [8] нетрудно установить следующее. Если $\lambda_{0}^{k}-$ простое собственное значение задачи (1.6), а $\psi_{0}^{k}$ - соответствующая собственная функция, то собственная функция $\psi_{\varepsilon}^{k}$ сходится к $\psi_{0}^{k}$. Если $\lambda_{0}=\lambda_{0}^{q}=\cdots=\lambda_{0}^{q+p-1}-p$-кратное собственное значение и $\lambda_{\varepsilon}^{k} \rightarrow \lambda_{0}^{k}$, $k=q, \ldots, q+p-1$, то к каждой соответствующей собственной функции $\psi_{0}^{k}$, $k=q, \ldots, q+p-1$, сходится линейная комбинация собственных функций $\psi_{\varepsilon}^{l}$, $l=q, \ldots, q+p-1$. Данная сходимость является сильной в $L_{2}(\Omega)$ и слабой в $H^{1}(\Omega)$, если предельная - третья краевая задача $(A>0)$, и сильной в $H^{1}(\Omega)$, если предельная - вторая краевая задача $(A=0)$.

Будем придерживаться обозначений из доказательства теоремы 1.4. Пусть $\lambda_{0}-$ простое собственное значение. Из леммы 2.6 и замечания 1.3 вытекает, что $\widehat{\psi}_{\varepsilon}$ сходится к $\psi_{0}$ в $L_{2}(\Omega)$.

Умножая (2.55) скалярно в $L_{2}(\Omega)$ на $\psi_{\varepsilon}$, благодаря леммам 2.3-2.5 видим, что

$$
\frac{1}{\lambda_{\varepsilon}-\widehat{\lambda}_{\varepsilon}} \int_{\Omega} f_{\varepsilon} \psi_{\varepsilon} d x=\left(\widehat{\psi}_{\varepsilon}, \psi_{\varepsilon}\right)_{L_{2}(\Omega)}=\left(\Psi_{0}+\varepsilon \Psi_{1}, \psi_{\varepsilon}\right)_{L_{2}(\Omega)}+O\left(\varepsilon^{3 / 2}(A+\mu)\right) .
$$

Из последнего соотношения, вводя обозначение

$$
\widetilde{\psi}_{\varepsilon}=\left(\Psi_{0}+\varepsilon \Psi_{1}, \psi_{\varepsilon}\right)_{L_{2}(\Omega)} \psi_{\varepsilon}
$$

и из $(2.55),(1.9)$ и следствия 2.1 выводим, что

$$
\left\|\widetilde{\psi}_{\varepsilon}-\widehat{\psi}_{\varepsilon}\right\|_{L_{2}(\Omega)} \leqslant C\left(\varepsilon^{3 / 2}(A+\mu)+\varepsilon^{1 / 2} \delta^{*}(\varepsilon)(A+\mu)^{2}\right)=o(\varepsilon(A+\mu)),
$$


откуда в силу замечания 1.3 и леммы 2.6 следует, что собственная функция $\widetilde{\psi}_{\varepsilon}$ возмушенной задачи, соответствуюшая $\lambda_{\varepsilon}$, сходится к $\psi_{0}$ в $L_{2}(\Omega)$ и имеет в силу лемм 2.3-2.5 и проведенного согласования следуюшую асимптотику в норме $H^{1}(\Omega)$ :

$$
\begin{aligned}
\widetilde{\psi}_{\varepsilon}(x)= & \left(\Psi_{0}(x, \mu, \varepsilon)+\varepsilon \Psi_{1}(x, \mu, \varepsilon)-\frac{\chi\left(\tau / c_{0}\right)}{\theta_{\varepsilon}^{\prime}(s)} \sum_{n=0}^{1} \varepsilon^{n+1} \Psi_{n}^{\nu}(s, \mu, \varepsilon) X(\xi)\right) \chi_{\varepsilon}(x) \\
& -\sum_{j=0}^{N-1} \frac{\chi\left(\left|\varsigma^{j}\right| \eta^{3 / 4}\right)}{\theta_{\varepsilon}^{\prime}(s)} \sum_{n=0}^{1} \varepsilon^{n+1} \Psi_{n}^{\nu}(s, \mu, \varepsilon) Y^{(j)}\left(\varsigma^{j}, \varepsilon\right)+o(\varepsilon(A+\mu)) .
\end{aligned}
$$

Пусть теперь $\lambda_{0}=\lambda_{0}^{q}=\cdots=\lambda_{0}^{q+p-1}-p$-кратное собственное значение. Вычислим коэффициенты линейной комбинации собственных функций возмущенной задачи, сходящихся к $\psi_{0}^{q}, \ldots, \psi_{0}^{q+p-1}$, и асимптотики этих линейных комбинаций. Прежде докажем вспомогательную лемму.

Лемма 2.7. Имеет место сходимость в $H^{1}(\Omega)$ :

$$
\sum_{n=q}^{q+p-1}\left(\psi_{0}^{k}, \Psi_{0}^{n}\right)_{L_{2}(\Omega)} \Psi_{0}^{n} \rightarrow \psi_{0}^{k}
$$

ДокаЗАТЕЛЬСТво. Собственные функции $\Psi_{0}^{k}, k=q, \ldots, q+p-1$, сходятся к собственным функциям $\psi_{0}^{k}$ задачи $(1.6)$ в том смысле, что для всякой собственной функции $\psi_{0}^{k}, k=q, \ldots, q+p-1$, существует линейная комбинация собственных функций $\Psi_{0}^{n}, n=q, \ldots, q+p-1$, сходящаяся к $\psi_{0}^{k}$ в $H^{1}(\Omega)[35]$ :

$$
\sum_{n=q}^{q+p-1} h_{n k} \Psi_{0}^{n}=\psi_{0}^{k}(1+o(1))
$$

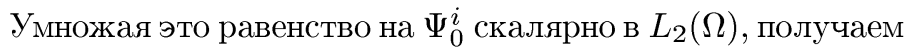

$$
h_{i k}=\left(\psi_{0}^{k}, \Psi_{0}^{i}\right)_{L_{2}(\Omega)}(1+o(1))
$$

откуда следует утверждение леммы. Лемма доказана.

Из формул (2.57) и лемм 2.3-2.5 вытекает, что

$$
h_{k i}^{\varepsilon}=\left(\Psi_{0}+\varepsilon \Psi_{1}, \psi_{\varepsilon}^{i}\right)_{L_{2}(\Omega)}+O\left(\varepsilon^{3 / 2}(A+\mu)\right),
$$

причем последние соотношения верны в предположении лишш ограниченности функции $\delta^{*}(\varepsilon)$. Используя эти соотношения и $(2.56)$, выводим оценку

$$
\left\|\sum_{i=q}^{q+p-1}\left(\Psi_{0}^{n}+\varepsilon \Psi_{1}^{n}, \psi_{\varepsilon}^{i}\right)_{L_{2}(\Omega)} \psi_{\varepsilon}^{i}-\widehat{\psi}_{\varepsilon}^{n}\right\|_{H^{1}(\Omega)}=O\left(\varepsilon^{3 / 2}(A+\mu)+\varepsilon^{1 / 2} \delta^{*}(\varepsilon)(A+\mu)^{2}\right),
$$


откуда в силу леммы 2.7 и оценки $\left\|\widehat{\psi}_{\varepsilon}^{n}-\Psi_{0}^{n}\right\|_{L_{2}(\Omega)}=o(1)$ (см. лемму 2.6) вытекает сходимость в $L_{2}(\Omega)$ :

$$
\widetilde{\psi}_{\varepsilon}^{k} \equiv \sum_{n=q}^{q+p-1}\left(\psi_{0}^{k}, \Psi_{0}^{n}\right)_{L_{2}(\Omega)} \sum_{i=q}^{q+p-1}\left(\Psi_{0}^{n}+\varepsilon \Psi_{1}^{n}, \psi_{\varepsilon}^{i}\right)_{L_{2}(\Omega)} \psi_{\varepsilon}^{i} \rightarrow \psi_{0}^{k},
$$

т. е. $\widetilde{\psi}_{\varepsilon}^{k}$ - линейная комбинация собственных функций возмущенной задачи, сходящаяся к $\psi_{0}^{k}$ в $L_{2}(\Omega)$. С другой стороны, в силу $(2.63)$ для $\widetilde{\psi}_{\varepsilon}^{k}$ верна оценка

$$
\left\|\widetilde{\psi}_{\varepsilon}^{k}-\sum_{i=q}^{q+p-1}\left(\psi_{0}^{k}, \Psi_{0}^{i}\right)_{L_{2}(\Omega)} \widehat{\psi}_{\varepsilon}^{i}\right\|_{H^{1}(\Omega)}=O\left(\varepsilon^{3 / 2}(A+\mu)+\varepsilon^{1 / 2} \delta^{*}(\varepsilon)(A+\mu)^{2}\right),
$$

откуда, учитывая равенство (1.9) и следствие 2.1 , получим, что асимптотика для $\widetilde{\psi}_{\varepsilon}^{k}$ в $H^{1}(\Omega)$ имеет следующий вид:

$$
\begin{aligned}
\widetilde{\psi}_{\varepsilon}^{k}(x) & =\sum_{i=q}^{q+p-1}\left(\psi_{0}^{k}, \Psi_{0}^{i}\right)_{L_{2}(\Omega)}\left(\left(\Psi_{0}^{i}(x, \mu, \varepsilon)+\varepsilon \Psi_{1}^{i}(x, \mu, \varepsilon)\right.\right. \\
- & \left.\frac{\chi\left(\tau / c_{0}\right)}{\theta_{\varepsilon}^{\prime}(s)} \sum_{n=0}^{1} \varepsilon^{n+1} \Psi_{n}^{i, \nu}(s, \mu, \varepsilon) X(\xi)\right) \chi_{\varepsilon}(x) \\
- & \left.\sum_{j=0}^{N-1} \frac{\chi\left(\left|\varsigma^{j}\right| \eta^{3 / 4}\right)}{\theta_{\varepsilon}^{\prime}(s)} \sum_{n=0}^{1} \varepsilon^{n+1} \Psi_{n}^{i, \nu}(s, \mu, \varepsilon) Y^{(j)}\left(\varsigma^{j}, \varepsilon\right)\right)+o(\varepsilon(A+\mu)) .
\end{aligned}
$$

Таким образом, доказана

ТЕОРема 2.1. Пусть выполнены условия теоремы 1.4. Если $\lambda_{0}=\lambda_{0}^{k}-$ простое собственное значение задачи (1.6), то собственная функиия $\widetilde{\psi}_{\varepsilon}$ из (2.60) при $\psi_{\varepsilon}=\psi_{\varepsilon}^{k}$ сходится $\kappa \psi_{0}^{k}$ в $L_{2}(\Omega)$-норме и имеет в $H^{1}(\Omega)$ асимптотику (2.61), где $\Psi_{1}$ - решение задачи (2.8), (2.28) при $\Psi_{0}=\Psi_{0}^{k}, \quad \Psi_{n}^{\nu}=\Psi_{n}^{k, \nu}$ значения нормальных производных функиий $\Psi_{n}^{k}$ на $\partial \Omega, X$ и $Y^{(j)}$ определеныс равенствами (2.13) и (2.25). Если $\lambda_{0}=\lambda_{0}^{q}=\cdots=\lambda_{0}^{q+p-1}-p$-кратное собственное значение задачи (1.6), то для каждой соответствующей собственной функиии $\psi_{0}^{k}, k=q, \ldots, q+p-1$, существует линейная комбинация (2.64) собственных функиий возмущенной задачи, сходящаяся $к \psi_{0}^{k}$ в норме $L_{2}(\Omega)$ и имеющая в $H^{1}(\Omega)$ асимптотику $(2.65)$.

Из доказательств теорем 1.4 и 2.1 следует справедливость следующего утверждения.

Лемма 2.8. Пусть выполнены условия (C0), (C1) и равенство (1.5) при $A \geqslant 0$ для функции $\eta$ из (C1). Тогда остатки в асимптотиках (1.10), (2.61) u (2.65) есть величины порядка $O\left(\varepsilon^{3 / 2}(A+\mu)+\varepsilon^{1 / 2} \delta^{*}(\varepsilon)(A+\mu)^{2}\right)$.

Если $A=0$, т.е. предельной является задача Неймана, то можно усилить утверждение теоремы 2.1 следуюшим образом. 
ТЕОРема 2.2. Пусть выполнены условия (C0), (C1) и равенство (1.5) при $A=0$ для функиии $\eta$ из (C1). Тогда собственная функиия $\widetilde{\psi}_{\varepsilon}^{k}$ из (2.60) в случае простого предельного собственного значения и линейная комбиначия собственных функиий $\widetilde{\psi}_{\varepsilon}^{k}$ из (2.64) в случае кратного предельного собственного значения сходятся $к$ предельным собственным функииям $\psi_{0}^{k}$ в $H^{1}(\Omega)$.

ДоказАТЕЛЬСтво. Докажем, что для всех $k$ при $\varepsilon \rightarrow 0$ имеет место равенство

$$
\left\|\widehat{\psi}_{\varepsilon}^{k}-\Psi_{0}^{k}\right\|_{H^{1}(\Omega)}=o(1)
$$

Поскольку

$$
\left\|\widehat{\psi}_{\varepsilon}^{k}-\Psi_{0}^{k}\right\|_{H^{1}(\Omega)}^{2}=\left\|\nabla_{x}\left(\widehat{\psi}_{\varepsilon}^{k}-\Psi_{0}^{k}\right)\right\|_{L_{2}(\Omega)}^{2}+\left\|\widehat{\psi}_{\varepsilon}^{k}-\Psi_{0}^{k}\right\|_{L_{2}(\Omega)}^{2},
$$

причем последнее слагаемое стремится к нулю при $\varepsilon \rightarrow 0$ в силу леммы 2.6, то остается оценить лишь норму градиента. Учитьвая вид $\widehat{\psi}_{\varepsilon}^{k}$, норму градиента $\widehat{\psi}_{\varepsilon}^{k}-\Psi_{0}^{k}$ оценим следуюшим образом:

$$
\begin{aligned}
\left\|\nabla_{x}\left(\widehat{\psi}_{\varepsilon}^{k}-\Psi_{0}^{k}\right)\right\|_{L_{2}(\Omega)}^{2} \leqslant & 2\left\|\nabla_{x}\left(\psi_{\varepsilon}^{\mathrm{ex}}-\Psi_{0}^{k}\right)\right\|_{L_{2}(\Omega)}^{2}+\sum_{j=0}^{N-1}\left\|\nabla_{x} \psi_{\varepsilon}^{\text {in }, j}\right\|_{L_{2}\left(\Omega_{j}^{\text {in }}\right)}^{2} \\
& +2\left\|\nabla_{x}\left(\psi_{\varepsilon}^{\mathrm{bl}} \chi\left(\tau / c_{0}\right)\right)\right\|_{L_{2}\left(\Omega_{\eta}^{\mathrm{bl}}\right)}^{2} \\
& +2 \sum_{j=0}^{N-1}\left\|\nabla_{x}\left(\psi_{\varepsilon}^{\text {mat }, j} \chi\left(\left|\varsigma^{j}\right| \eta^{3 / 4}\right)\right)\right\|_{L_{2}\left(\Omega_{j}^{\text {mat }}\right)}^{2},
\end{aligned}
$$

где $\psi_{\varepsilon}^{\mathrm{ex}}, \psi_{\varepsilon}^{\mathrm{bl}}, \psi_{\varepsilon}^{\mathrm{in}, j}$ и $\psi_{\varepsilon}^{\mathrm{mat}, j}$ - функции, определенные в доказательстве теоремы 1.4 и соответствующие $\psi_{0}^{k}$.

В силу леммы 2.3 и определения $\psi_{\varepsilon}^{\mathrm{ex}}$ имеем

$$
\left\|\nabla_{x}\left(\psi_{\varepsilon}^{\mathrm{ex}}-\Psi_{0}^{k}\right)\right\|_{L_{2}(\Omega)}^{2} \leqslant C \varepsilon^{2} \mu^{4} .
$$

Нетрудно видеть, что

$$
\left\|\nabla_{x}\left(\psi_{\varepsilon}^{\mathrm{bl}} \chi\left(\tau / c_{0}\right)\right)\right\|_{L_{2}\left(\Omega_{\eta}^{\mathrm{bl}}\right)}^{2} \leqslant C\left(\left\|\nabla_{x} \psi_{\varepsilon}^{\mathrm{bl}}\right\|_{L_{2}\left(\Omega_{\eta}^{\mathrm{bl}} \cap \Omega^{\mathrm{bl}}\right)}^{2}+\left\|\psi_{\varepsilon}^{\mathrm{bl}}\right\|_{L_{2}\left(\Omega^{\mathrm{bl}}\right)}^{2}\right) .
$$

Второе слагаемое в правой части полученного неравенства оценивается сверху величиной $C \varepsilon^{3 / 2} \mu$ (см. лемму 2.4). Прямыми вычислениями с использованием явного вида функций $v_{i}$, ограниченности функции $\delta^{*}(\varepsilon)$, леммы 2.3 и равенства $N=2 \varepsilon^{-1}$ проверяется, что

$$
\left\|\nabla_{x} \psi_{\varepsilon}^{\mathrm{bl}}\right\|_{L_{2}\left(\Omega_{\eta}^{\left.\mathrm{bl} \cap \Omega^{\mathrm{bl}}\right)}\right.}^{2} \leqslant C \sum_{j=0}^{N-1}\left(\left\|\nabla_{\xi} \psi_{\varepsilon}^{\mathrm{bl}}\right\|_{L_{2}\left(\Pi_{\eta}^{(j)}\right)}^{2}+\varepsilon^{2}\left\|\frac{\partial}{\partial s} \psi_{\varepsilon}^{\mathrm{bl}}\right\|_{L_{2}\left(\Pi^{(j)}\right)}^{2}\right) \leqslant C \mu .
$$

Таким образом,

$$
\left\|\nabla_{x}\left(\psi_{\varepsilon}^{\mathrm{bl}} \chi\left(\tau / c_{0}\right)\right)\right\|_{L_{2}\left(\Omega_{\eta}^{\mathrm{bl}}\right)}^{2} \leqslant C \mu .
$$


Из явного вида функций $\psi_{\varepsilon}^{\text {in, } j}$ следует, что

$$
\sum_{j=0}^{N-1}\left\|\nabla_{x} \psi_{\varepsilon}^{\text {in }, j}\right\|_{L_{2}\left(\Omega_{j}^{\text {in }}\right)}^{2} \leqslant C \mu .
$$

Используя асимптотику (2.52), докажем, что

$$
\sum_{j=0}^{N-1}\left\|\nabla_{x}\left(\psi_{\varepsilon}^{\mathrm{mat}, j} \chi\left(\left|\varsigma^{j}\right| \eta^{3 / 4}\right)\right)\right\|_{L_{2}\left(\Omega_{j}^{\mathrm{mat}}\right)}^{2} \leqslant C \eta^{1 / 5} .
$$

Из оценок (2.67)-(2.71) выводим равенство (2.66). Заметим, что сходимость (2.66) получена без использования равенства (1.9) и верна для любой ограниченной функции $\delta^{*}(\varepsilon)$.

Пусть $\psi_{0}$ соответствует простому собственному значению. Ввиду сходимостей (2.66) и $\Psi_{0} \stackrel{H^{1}(\Omega)}{\longrightarrow} \psi_{0}$ (см. замечание 1.3$)$ заключаем, что $\widehat{\psi}_{\varepsilon}$ сходится к $\psi_{0}$ сильно в $H^{1}(\Omega)$. Следовательно, собственная функция $\widetilde{\psi}_{\varepsilon}$ из $(2.60)$ в силу леммы 2.8 удовлетворяет равенству

$$
\left\|\widetilde{\psi}_{\varepsilon}-\widehat{\psi}_{\varepsilon}\right\|_{H^{1}(\Omega)}=o(1),
$$

откуда следует, что собственная функция $\psi_{\varepsilon}$ сходится к $\psi_{0}$ в норме $H^{1}(\Omega)$.

Пусть $\psi_{0}$ соответствует $p$-кратному собственному значению

$$
\lambda_{0}=\lambda_{0}^{q}=\cdots=\lambda_{0}^{q+p-1},
$$

которому соответствуют собственные функции $\psi_{0}^{k}, k=q, \ldots, q+p-1$. Для функций $\widehat{\psi}_{\varepsilon}^{k}$ и $\Psi_{0}^{k}$ выполнено соотношение (2.66). Из этих соотношений, леммы 2.7 и оценки (2.63) следует, что линейная комбинация собственных функций возмущенной задачи $\widetilde{\psi}_{\varepsilon}^{k}$ из $(2.64)$ сходится к $\psi_{0}^{k}$ в $H^{1}(\Omega)$. Теорема доказана.

\section{§3. Асимптотики собственных элементов возмущенной задачи в условиях теоремы 1.5}

В настоящем параграфе будут получены асимптотики собственных элементов возмушенной задачи в случае нарушения условия (1.9) теоремы 1.4. Вначале докажем теорему 1.5 об асимптотиках собственных значений, а затем - теорему 3.1 об асимптотиках соответствуюших собственных функций. Далее, если не оговорено особо, придерживаемся обозначений из предыдушего параграфа.

ДОКАЗАТЕЛЬСТВо ТЕОРЕМЫ 1.5. В доказательстве используем ограниченность функции $\delta^{*}(\varepsilon)$, доказанную в следствии 2.2 . В $\S 6$ покажем, что собственные значения задачи (1.7), (1.8) удовлетворяют следуюшим асимптотическим формулам:

$$
\Lambda_{0}^{k}(\mu, \varepsilon)=\lambda_{0}^{k}+\mu \int_{\partial \Omega}\left(\psi_{0}^{k}\right)^{2} \theta_{0}^{\prime} d s+O\left(\mu^{2}+(A+\mu) \sigma\right),
$$

где в случае кратного собственного значения $\lambda_{0}^{k}$ соответствуюшие собственные функции $\psi_{0}^{k}$ дополнительно предполагаются ортогональными в $L_{2}(\partial \Omega)$ с весом $\theta_{0}^{\prime}$, $\sigma=\sigma(\varepsilon)=\left\|\theta_{\varepsilon}^{\prime}-\theta_{0}^{\prime}\right\|_{C(\partial \Omega)}=o(1)$. Из лемм $2.3,2.8$ и следствия 2.2 выводим, что 
$\left|\lambda_{\varepsilon}^{k}-\Lambda_{0}^{k}\right|=O\left(\varepsilon^{3 / 2}(A+\mu)+\varepsilon^{1 / 2}(A+\mu)^{2}\right)$, откуда в силу асимптотик (3.1) следует утверждение доказываемой теоремы. Теорема доказана.

Выведем теперь асимптотики собственных функций возмущенной задачи в условиях теоремы 1.5. Пусть вначале $\lambda_{0}$ - простое собственное значение. Из (2.60) и лемм $2.3,2.8$ вытекает, что собственная функция возмущенной задачи

$$
\widetilde{\psi}_{\varepsilon}=\left(\Psi_{0}, \psi_{\varepsilon}\right)_{L_{2}(\Omega)} \psi_{\varepsilon},
$$

соответствуюшая $\lambda_{\varepsilon} \underset{\varepsilon \rightarrow 0}{\longrightarrow} \lambda_{0}$, удовлетворяет оценке

$$
\left\|\widetilde{\psi}_{\varepsilon}-\widehat{\psi}_{\varepsilon}\right\|_{H^{1}(\Omega)}=O\left(\varepsilon^{3 / 2}(A+\mu)+\varepsilon^{1 / 2}(A+\mu)^{2}\right) .
$$

Прямыми вычислениями с использованием лемм $2.3-2.5$ и результатов согласования из предыдущего параграфа нетрудно проверить, что $H^{1}(\Omega)$-норма функции

$$
\left(\varepsilon \Psi_{1}-\varepsilon^{2} \frac{\chi\left(\tau / c_{0}\right)}{\theta_{\varepsilon}^{\prime}} \Psi_{1}^{\nu} X\right) \chi_{\varepsilon}-\varepsilon^{2} \sum_{j=0}^{N-1} \frac{\chi\left(\left|\varsigma^{j}\right| \eta^{3 / 4}\right)}{\theta_{\varepsilon}^{\prime}} \Psi_{1}^{\nu} Y^{(j)}
$$

есть величина порядка $O\left(\varepsilon^{1 / 2}(A+\mu)\right)$. Следовательно, функция $\widetilde{\psi}_{\varepsilon}$ из $(3.2)$ сходится к $\psi_{0}$ в $L_{2}(\Omega)$ и имеет следуюшую асимптотику в норме $H^{1}(\Omega)$ :

$$
\begin{aligned}
\widetilde{\psi}_{\varepsilon}(x)= & \left(\Psi_{0}(x, \mu, \varepsilon)+\varepsilon \frac{\chi\left(\tau / c_{0}\right)}{\theta_{\varepsilon}^{\prime}(s)} \Psi_{0}^{\nu}(s, \mu, \varepsilon) X(\xi)\right) \chi_{\varepsilon}(x) \\
& +\varepsilon \sum_{j=0}^{N-1} \frac{\chi\left(\left|\varsigma^{j}\right| \eta^{3 / 4}\right)}{\theta_{\varepsilon}^{\prime}(s)} \Psi_{0}^{\nu}(s, \mu, \varepsilon) Y^{(j)}\left(\varsigma^{j}, \varepsilon\right)+O\left(\varepsilon^{1 / 2}(A+\mu)\right) .
\end{aligned}
$$

Пусть теперь $\lambda_{0}=\lambda_{0}^{q}=\cdots=\lambda_{0}^{q+p-1}-p$-кратное собственное значение. Из (2.64) и лемм $2.3,2.8$ выводим, что линейная комбинация собственных функций возмушенной задачи

$$
\widetilde{\psi}_{\varepsilon}^{k} \equiv \sum_{n=q}^{q+p-1}\left(\psi_{0}^{k}, \Psi_{0}^{n}\right)_{L_{2}(\Omega)} \sum_{i=q}^{q+p-1}\left(\Psi_{0}^{n}, \psi_{\varepsilon}^{i}\right)_{L_{2}(\Omega)} \psi_{\varepsilon}^{i}
$$

сходится в $L_{2}(\Omega)$ к $\psi_{0}^{k}, k=q, \ldots, q+p-1$, и имеет в норме $H^{1}(\Omega)$ асимптотику

$$
\begin{aligned}
\widetilde{\psi}_{\varepsilon}^{k}(x)= & \sum_{i=q}^{q+p-1}\left(\psi_{0}^{k}, \Psi_{0}^{i}\right)_{L_{2}(\Omega)}\left(\left(\Psi_{0}^{i}(x, \mu, \varepsilon)+\varepsilon \frac{\chi\left(\tau / c_{0}\right)}{\theta_{\varepsilon}^{\prime}(s)} \Psi_{0}^{i, \nu}(s, \mu, \varepsilon) X(\xi)\right) \chi_{\varepsilon}(x)\right. \\
& \left.+\varepsilon \sum_{j=0}^{N-1} \frac{\chi\left(\left|\varsigma^{j}\right| \eta^{3 / 4}\right)}{\theta_{\varepsilon}^{\prime}(s)} \Psi^{i, \nu}(s, \mu, \varepsilon) Y^{(j)}\left(\varsigma^{j}, \varepsilon\right)\right)+O\left(\varepsilon^{1 / 2}(A+\mu)\right)
\end{aligned}
$$

Как и в случае простого предельного собственного значения, мы учли, что $H^{1}(\Omega)$-норма некоторых слагаемых в правой части (2.65) есть величина порядка $O\left(\varepsilon^{1 / 2}(A+\mu)\right)$.

В силу леммы 2.3 и теоремы 2.2 следует, что утверждение теоремы 2.2 имеет место и для функций (3.2), (3.4).

Таким образом, доказана 
ТЕОРема 3.1. Пусть выполнены условия теоремы 2.2. Если $\lambda_{0}=\lambda_{0}^{k}-$ простое собственное значение задачи (1.6), то собственная функиия $\widetilde{\psi}_{\varepsilon}$ из $(3.2)$, где $\psi_{\varepsilon}=\psi_{\varepsilon}^{k}, \Psi_{0}=\Psi_{0}^{k}$, сходится $к \psi_{0}^{k}$ в $L_{2}(\Omega)$ при $A \geqslant 0$ и в $H^{1}(\Omega)$ nри $A=0$ и имеет в $H^{1}(\Omega)$-норме асимптотику (3.3).

Если $\lambda_{0}=\lambda_{0}^{q}=\cdots=\lambda_{0}^{q+p-1}-p$-кратное собственное значение, то для каждой соответствующей собственной функиии $\psi_{0}^{k}, k=q, \ldots, k+$ $p-1$, существует линейная комбинация (3.4), сходящаяся $к \psi_{0}^{k}$ в $L_{2}(\Omega)$ nри $A \geqslant 0$ и в $H^{1}(\Omega)$ при $A=0$ и имеющая в $H^{1}(\Omega)$-норме асимптотики (3.5). В асимптотиках (3.3), (3.5) обозначения те же, что и в теореме 2.1.

\section{§4. Вспомогательное утверждение}

В настоящем параграфе докажем вспомогательную лемму, которая будет использована в следующем параграфе при доказательстве теоремы 1.1. Сформулируем указанную лемму.

ЛЕмма 4.1. Пусть выполнены условия (C0) и (C1), функция $\eta(\varepsilon)$ из $(\mathrm{C} 1)$ ограничена сверху числом $\pi / 2$ и удовлетворяет соотношению (1.3), и для всех $i, j$ и с справедливы равенства а $a^{j}(\varepsilon)+b^{j}(\varepsilon)=2 \eta(\varepsilon), a^{i}(\varepsilon)=a^{j}(\varepsilon), b^{i}(\varepsilon)=$ $b^{j}(\varepsilon)$. Пусть также существует фиксированное число $c_{3}>0$, для которого норма Гёльдера $\left\|\theta_{\varepsilon}^{\prime}\right\|_{C^{3+c_{3}(\partial \Omega)}}$ ограничена по $\varepsilon$. Тогда собственное значение $\lambda_{\varepsilon}^{k}$ возмущенной задачи сходится $\kappa$ собственному значению $\lambda_{0}^{k}$ предельной задачи (1.4) и имеет асимптотику

$$
\lambda_{\varepsilon}^{k}=\lambda_{0}^{k}+\varepsilon \ln \sin \eta(\varepsilon) \int_{\partial \Omega}\left(\frac{\partial \psi_{0}^{k}}{\partial \nu}\right)^{2} \frac{d s}{\theta_{\varepsilon}^{\prime}}+O\left(\varepsilon^{3 / 2}\left(|\ln \eta(\varepsilon)|^{3 / 2}+1\right)\left(\frac{\pi}{2}-\eta(\varepsilon)\right)\right) .
$$

ДокАЗАТЕЛЬСТво. Сходимость собственных значений доказывается аналогично работам [2], [6], [8]. Доказательство асимптотик проведем по схеме, использованной в $\S 2$. Как и ранее, вначале формально построим асимптотики, а затем дадим их строгое обоснование. Следует также отметить, что формальное построение асимптотик будем проводить по схеме, предложенной в работах [17], [18]. Отличием является оценка остатка в асимптотиках одновременно по двум параметрам $\varepsilon$ и $\eta$, а также чуть более общая постановка рассматриваемой здесь задачи по сравнению с [17], [18] и отказ от дополнительных ограничений, сделанных в [17], [18]. Подробно остановимся только на случае простого предельного собственного значения; случай кратного собственного значения доказывается аналогично.

Пусть $\lambda_{0}$ - простое собственное значение предельной задачи (1.4), $\psi_{0}$ - соответствующая (нормированная в $L_{2}(\Omega)$ ) собственная функция, $\lambda_{\varepsilon}-$ собственное значение возмушенной задачи, сходящееся к $\lambda_{0}$.

Асимптотику собственного значения $\lambda_{\varepsilon}$ будем искать в виде

$$
\lambda_{\varepsilon}=\lambda_{0}+\varepsilon \lambda_{1}(\varepsilon) \ln \sin \eta,
$$

а асимптотика соответствуюшей собственной функции строится как сумма внешнего разложения и пограничного слоя:

$$
\begin{aligned}
\psi_{\varepsilon}(x) & =\psi_{\varepsilon}^{\mathrm{ex}}(x, \eta)+\chi\left(\tau / c_{0}\right) \psi_{\varepsilon}^{\mathrm{bl}}(\xi, s, \eta), \\
\psi_{\varepsilon}^{\mathrm{ex}}(x, \eta) & =\psi_{0}(x)+\varepsilon \psi_{1}(x, \varepsilon) \ln \sin \eta, \\
\psi_{\varepsilon}^{\mathrm{bl}}(\xi, s, \eta) & =\varepsilon v_{1}(\xi, s, \varepsilon, \eta)+\varepsilon^{2} v_{2}(\xi, s, \varepsilon, \eta),
\end{aligned}
$$


где $\xi=\left(\xi_{1}, \xi_{2}\right), \xi_{1}=\left(\theta_{\varepsilon}(s)-\theta_{\varepsilon}\left(s_{0}^{\varepsilon}\right)\right) / \varepsilon-\left(b^{j}(\varepsilon)-a^{j}(\varepsilon)\right) / 2, \xi_{2}=\tau \theta_{\varepsilon}^{\prime}(s) / \varepsilon$. Заметим также, что построение асимптотик здесь удается провести без использования метода согласования асимптотических разложений.

Подставим (4.1) и (4.2) в уравнение (1.1) и выпишем коэффициенты при $\varepsilon \ln \sin \eta$ :

$$
\left(\Delta+\lambda_{0}\right) \psi_{1}=-\lambda_{1} \psi_{0}, \quad x \in \Omega .
$$

Подставляя (4.1) и (4.3) в уравнение (1.1), получим уравнения (2.9) и (2.10) для функций $v_{1}$ и $v_{2}$. Граничные условия для этих функций выводим из требования того, что сумма правых частей (4.2) и (4.3) удовлетворяет обоим граничным условиям из (1.2):

$$
\begin{gathered}
v_{1}=-\psi_{1}^{D} \ln \sin \eta, \quad \xi \in \gamma^{\eta}, \quad \frac{\partial v_{1}}{\partial \xi_{2}}=\frac{1}{\theta_{\varepsilon}^{\prime}} \psi_{0}^{\nu}, \quad \xi \in \Gamma^{\eta}, \\
\frac{\partial v_{2}}{\partial \xi_{2}}=\frac{1}{\theta_{\varepsilon}^{\prime}} \psi_{1}^{\nu} \ln \sin \eta, \quad \xi \in \Gamma^{\eta},
\end{gathered}
$$

где $\gamma^{\eta}$ - объединение интервалов $(\pi j-\eta, \pi j+\eta), j \in \mathbb{Z}$, лежаших на оси $\xi_{1}$, а $\Gamma^{\eta}$ - дополнение $\bar{\gamma}^{\eta}$ до оси $\xi_{1}, \psi_{1}^{D}$ и $\psi_{i}^{\nu}$ - значения функций $\psi_{i}$ и их нормальных производных на границе $\partial \Omega$. Задача $(2.9),(4.5)$ решается явно:

$$
\begin{gathered}
v_{1}(\xi, s, \varepsilon, \eta)=-\frac{1}{\theta_{\varepsilon}^{\prime}(s)} \psi_{0}^{\nu}(s) X_{\eta}(\xi), \\
X_{\eta}(\xi)=\operatorname{Re} \ln \left(\sin z+\sqrt{\sin ^{2} z-\sin ^{2} \eta}\right)-\xi_{2} .
\end{gathered}
$$

Нетрудно проверить, что $X_{\eta} \in \mathscr{V}_{\eta} \cap H^{1}\left(\Pi^{(j)}\right)$ - четная по $\xi_{1}$ гармоническая функция, где $\mathscr{V} \eta$ - пространство $\pi$-периодических по переменной $\xi_{1}$, экспоненциально убывающих вместе со всеми своими производными при $\xi_{2} \rightarrow+\infty$ равномерно по $\xi_{1}$ функций, принадлежаших $C^{\infty}\left(\left\{\xi: \xi_{2}>0\right\} \cup \gamma^{\eta} \cup \Gamma^{\eta}\right)$. Функция $X_{\eta}$ удовлетворяет граничным условиям

$$
X_{\eta}(\xi)=\ln \sin \eta, \quad \xi \in \gamma^{\eta}, \quad \frac{\partial X}{\partial \xi_{2}}=-1, \quad \xi \in \Gamma^{\eta} .
$$

Функция $v_{1}$, определенная равенством (4.7), в силу (4.8) удовлетворяет граничному условию

$$
v_{1}=-\frac{1}{\theta_{\varepsilon}^{\prime}} \psi_{0}^{\nu} \ln \sin \eta, \quad \xi \in \gamma^{\eta},
$$

из сравнения которого с (4.5) получаем краевое условие для $\psi_{1}$ :

$$
\psi_{1}=\frac{1}{\theta_{\varepsilon}^{\prime}} \frac{\partial \psi_{0}}{\partial \nu}, \quad x \in \partial \Omega
$$

Условие разрешимости краевой задачи (4.4), (4.9) приводит к формуле для $\lambda_{1}$ :

$$
\lambda_{1}=\int_{\partial \Omega}\left(\frac{\partial \psi_{0}}{\partial \nu}\right)^{2} \frac{d s}{\theta_{\varepsilon}^{\prime}(s)} .
$$


Функцию $\psi_{1}$ выберем ортогональной $\psi_{0}$ в $L_{2}(\Omega)$. Функция $v_{2}$ определяется следуюшим образом:

$$
v_{2}=\frac{\psi_{0}^{\nu}}{2\left(\theta_{\varepsilon}^{\prime}\right)^{2}} \xi_{2}^{2}\left(\frac{\theta_{\varepsilon}^{\prime \prime}}{\theta_{\varepsilon}^{\prime}} \frac{\partial X_{\eta}}{\partial \xi_{1}}+K \frac{\partial X_{\eta}}{\partial \xi_{2}}\right)-\frac{2}{\theta_{\varepsilon}^{\prime}}\left(\frac{\psi_{0}^{\nu}}{\theta_{\varepsilon}^{\prime}}\right)^{\prime} v_{2}^{\text {odd }}-\frac{1}{\theta_{\varepsilon}^{\prime}} \psi_{1}^{\nu} \ln \sin \eta X_{\eta},
$$

где $v_{2}^{\text {odd }}$ - экспоненциально убываюшее решение краевой задачи

$$
\Delta_{\xi} v_{2}^{\text {odd }}=\frac{\partial X_{\eta}}{\partial \xi_{1}}, \quad \xi_{2}>0, \quad v_{2}^{\text {odd }}=0, \quad \xi \in \gamma^{\eta}, \quad \frac{\partial v_{2}^{\text {odd }}}{\partial \xi_{2}}=0, \quad \xi \in \Gamma^{\eta} .
$$

Сушествование решения задачи (4.12), а также его нечетность по $\xi_{1}$ и принадлежность $\mathscr{V}_{\eta} \cap H^{1}\left(\Pi^{(j)}\right)$ были доказаны в [17].

Для обоснования формально построенных асимптотик понадобятся следующие леммы.

ЛЕмма 4.2. Выполняются следующие свойства:

1) для цельх $m \geqslant 0$ справедливы неравенства

$$
\left\|\xi_{2}^{m} X_{\eta}\right\|_{L_{2}\left(\Pi^{(j)}\right)} \leqslant C\left(\frac{\pi}{2}-\eta\right)^{2}\left(\left|\ln \left(\frac{\pi}{2}-\eta\right)\right|^{1 / 2}+1\right),
$$

где константы $C$ не зависят от $\eta$;

2) для целых $m, p \geqslant 0$ имеют место оценки

$$
\begin{aligned}
&\left\|\xi_{2}^{m} \nabla_{\xi} \frac{\partial^{m} X_{\eta}}{\partial \xi_{2}^{m}}\right\|_{L_{2}\left(\Pi^{(j)}\right)} \leqslant C|\ln \sin \eta|^{1 / 2} \\
&\left\|\xi_{2}^{m+p+1} \nabla_{\xi} \frac{\partial^{m} X_{\eta}}{\partial \xi_{2}^{m}}\right\|_{L_{2}\left(\Pi^{(j)}\right)} \leqslant C\left(\frac{\pi}{2}-\eta\right)^{2}\left(\left|\ln \left(\frac{\pi}{2}-\eta\right)\right|^{1 / 2}+1\right)
\end{aligned}
$$

где константы $C$ не зависят от $\eta$.

ДокАЗАТЕльСтво. Вначале докажем утверждение 1) для $m=0 . \mathrm{B}[20, \S 3]$ было показано, что $\left\|X_{\eta}\right\|_{L_{2}\left(\Pi^{(j)}\right)}-$ непрерывная функция по $\eta \in[0, \pi / 2]$. Для доказательства требуемой оценки выясним поведение этой функции при $\eta \rightarrow \pi / 2$. Непосредственно проверяется, что функция

$$
X_{\eta}^{1}(\xi)=-\frac{1}{2} \xi_{2} \int_{\xi_{2}}^{+\infty} X_{\eta}\left(\xi_{1}, t\right) d t
$$

является четной по $\xi_{1}$, принадлежит $\mathscr{V}_{\eta}$ и в области $\xi_{2}>0$ является решением уравнения $\Delta_{\xi} X_{\eta}^{1}(\xi)=X_{\eta}$, удовлетворяюшим граничным условиям

$$
X_{\eta}^{1}=0, \quad \frac{\partial X_{\eta}^{1}}{\partial \xi_{2}}=-\frac{1}{2} \int_{0}^{+\infty} X_{\eta}\left(\xi_{1}, t\right) d t, \quad \xi_{2}=0 .
$$

Используя перечисленные свойства функций $X_{\eta}^{1}$ и $X_{\eta}$ и доказанное в $[20, \S 3]$ равенство

$$
\int_{\Pi^{(j)}} X_{\eta}^{2} d \xi=\int_{\Pi^{(j)}}\left(X_{\eta}+\xi_{2}-\ln \sin \eta\right) X_{\eta} d \xi
$$


интегрируя по частям, получим

$$
\begin{aligned}
\int_{\Pi^{(j)}} X_{\eta}^{2} d \xi & =\int_{\Pi^{(j)}}\left(X_{\eta}+\xi_{2}-\ln \sin \eta\right) \Delta_{\xi} X_{\eta}^{1} d \xi \\
& =\int_{\eta}^{\pi / 2}\left(X_{\eta}\left(\xi_{1}, 0\right)-\ln \sin \eta\right) \int_{0}^{+\infty} X_{\eta}\left(\xi_{1}, t\right) d t d \xi_{1}
\end{aligned}
$$

Поскольку при $\xi_{1} \in(\eta, \pi / 2]$

$$
\frac{d^{2}}{d \xi_{1}^{2}} \int_{0}^{+\infty} X_{\eta}\left(\xi_{1}, t\right) d t=-\int_{0}^{+\infty} \frac{\partial^{2}}{\partial t^{2}} X_{\eta}\left(\xi_{1}, t\right) d t=-1
$$

то ввиду четности и $\pi$-периодичности $X_{\eta}$ по $\xi_{1}$ получаем

$$
\int_{0}^{+\infty} X_{\eta}\left(\xi_{1}, t\right) d t=-\frac{1}{2}\left(\xi_{1}-\frac{\pi}{2}\right)^{2}+\int_{0}^{+\infty} X_{\eta}\left(\frac{\pi}{2}, t\right) d t .
$$

Применяя оценку $|\ln (1+a)| \leqslant a, a \geqslant 0$, к подынтегральной функции

$$
X_{\eta}\left(\frac{\pi}{2}, t\right)=\ln \left(1+\frac{e^{-2 t}-1+\sqrt{\left(1-e^{-2 t}\right)^{2}+4 e^{-2 t} \cos ^{2} \eta}}{2}\right)
$$

из правой части равенства (4.14) и вычисляя полученный интеграл, выводим соотношение $(\eta \rightarrow \pi / 2)$

$$
\int_{0}^{+\infty} X_{\eta}\left(\frac{\pi}{2}, t\right) d t=O\left(\eta_{1}^{2} \ln \eta_{1}\right)
$$

где $\eta_{1}=\pi / 2-\eta$. В работе [17] было доказано, что

$$
\int_{\gamma^{\eta} \cap \bar{\Pi}} \frac{\partial X_{\eta}}{\partial \xi_{2}} d \xi_{1}=\pi-2 \eta, \quad \int_{\Gamma^{\eta} \cap \bar{\Pi}} X_{\eta} d \xi_{1}=-2 \eta \ln \sin \eta .
$$

Подставляя теперь (4.14)-(4.16) в (4.13), приходим к равенствам $(\eta \rightarrow \pi / 2)$

$$
\begin{aligned}
\int_{\Pi^{(j)}} X^{2} d \xi= & -\frac{1}{2} \int_{\eta}^{\pi / 2} \ln \left(\frac{\sin \xi_{1}}{\sin \eta}+\sqrt{\frac{\sin ^{2} \xi_{1}}{\sin ^{2} \eta}-1}\right)\left(\xi_{1}-\frac{\pi}{2}\right)^{2} d \xi_{1} \\
& +\frac{1}{2} \int_{0}^{+\infty} X_{\eta}\left(\frac{\pi}{2}, \xi_{2}\right) d \xi_{2} \int_{\eta}^{\pi / 2}\left(X_{\eta}\left(\xi_{1}, 0\right)-\ln \sin \eta\right) d \xi_{1} \\
= & -\frac{1}{2} \int_{0}^{\eta_{1}} t^{2}\left(\ln \left(\cos t+\sqrt{\cos ^{2} t-\sin ^{2} \eta}\right)-\ln \sin \eta\right) d t+O\left(\eta_{1}^{4} \ln \eta_{1}\right) \\
= & O\left(\eta_{1}^{4} \ln \eta_{1}\right) .
\end{aligned}
$$

Здесь была сделана замена $t=\pi / 2-\xi_{1}$. Из полученной оценки для $\left\|X_{\eta}\right\|_{L_{2}\left(\Pi^{(j)}\right)}^{2}$ и непрерывности этой функции по $\eta \in[0, \pi / 2]$ вытекает утверждение 1$)$ для $m=0$.

Из явного вида функции $X$, ее бесконечной дифференщируемости по $(\xi, \eta)$ при $\xi_{2} \geqslant 1$, непрерывности по $(\xi, \eta) \in\left\{\xi: \xi_{2}>0\right\} \times(0, \pi / 2]$ и экспоненциального убывания при $\xi_{2} \rightarrow+\infty$ следует, что для $m \geqslant 1$ норма $\left\|\xi_{2}^{m} X_{\eta}\right\|_{L_{2}\left(\Pi^{(j)}\right)}-$ непрерывная по $\eta \in(0, \pi / 2]$ функция и верна оценка

$$
\left\|\xi_{2}^{m} X_{\eta}\right\|_{L_{2}\left(\Pi^{(j)} \cap\left\{\xi: \xi_{2}>1\right\}\right)} \leqslant C \eta_{1}^{2},
$$


где константа $C$ не зависит от $\eta$. Тогда в силу неравенства

$$
\left\|\xi_{2}^{m} X_{\eta}\right\|_{L_{2}\left(\Pi^{(j)} \cap\left\{\xi: \xi_{2}<1\right\}\right)}<\left\|X_{\eta}\right\|_{L_{2}\left(\Pi^{(j)}\right)} \leqslant C \eta_{1}^{2}\left(\left|\ln \eta_{1}\right|^{1 / 2}+1\right)
$$

и утверждения 1 ) для $m=0$ заключаем, что утверждение 1$)$ верно и для $m>0$.

Проведем интегрирование по частям в равенстве $\int_{\Pi} X_{\eta} \Delta_{\xi} X_{\eta} d \xi=0$; в результате получим

$$
\int_{\Pi}\left|\nabla_{\xi} X_{\eta}\right|^{2} d \xi=-\ln \sin \eta \int_{\gamma^{\eta} \cap \bar{\Pi}^{(j)}} \frac{\partial X_{\eta}}{\partial \xi_{2}} d \xi_{1}+\int_{\Gamma^{\eta} \cap \bar{\Pi}^{(j)}} X_{\eta} d \xi_{1},
$$

откуда в силу (4.16) вытекает

$$
\left\|\nabla_{\xi} X_{\eta}\right\|_{L_{2}\left(\Pi^{(j)}\right)}=\pi^{1 / 2}|\ln \sin \eta|^{1 / 2} .
$$

Из цепочки равенств $(m \geqslant 0, p \geqslant 0, m+p \geqslant 1, m, p \in \mathbb{Z})$

$$
\begin{aligned}
0 & =\int_{\Pi^{(j)}} \xi_{2}^{2(m+p)} \frac{\partial^{m} X_{\eta}}{\partial \xi_{2}^{m}} \Delta_{\xi} \frac{\partial^{m} X_{\eta}}{\partial \xi_{2}^{m}} d \xi \\
& =-\int_{\Pi^{(j)}} \xi_{2}^{2(m+p)}\left|\nabla_{\xi} \frac{\partial^{m} X_{\eta}}{\partial \xi_{2}^{m}}\right|^{2} d \xi-2(m+p) \int_{\Pi^{(j)}} \xi_{2}^{2(m+p)-1} \frac{\partial^{m} X_{\eta}}{\partial \xi_{2}^{m}} \frac{\partial^{m+1} X_{\eta}}{\partial \xi_{2}^{m+1}} d \xi \\
& =-\left\|\xi_{2}^{m+p} \nabla_{\xi} \frac{\partial^{m} X_{\eta}}{\partial \xi_{2}^{m}}\right\|_{L_{2}\left(\Pi^{(j)}\right)}^{2}+(m+p)(2(m+p)-1)\left\|\xi_{2}^{m+p-1} \frac{\partial^{m} X_{\eta}}{\partial \xi_{2}^{m}}\right\|_{L_{2}\left(\Pi^{(j)}\right)}^{2}
\end{aligned}
$$

выводим формулы

$$
\left\|\xi_{2}^{m+p} \nabla_{\xi} \frac{\partial^{m} X_{\eta}}{\partial \xi_{2}^{m}}\right\|_{L_{2}\left(\Pi^{(j)}\right)}=\sqrt{(m+p)(2(m+p)-1)}\left\|\xi_{2}^{m+p-1} \frac{\partial^{m} X_{\eta}}{\partial \xi_{2}^{m}}\right\|_{L_{2}\left(\Pi^{(j)}\right)} .
$$

Применяя данные формулы при $p=0, m \geqslant 1$ и при $p \geqslant 1, m \geqslant 0$, получаем оценки

$$
\begin{aligned}
\left\|\xi_{2}^{m} \nabla_{\xi} \frac{\partial^{m} X_{\eta}}{\partial \xi_{2}^{m}}\right\|_{L_{2}\left(\Pi^{(j)}\right)} & \leqslant C\left\|\nabla_{\xi} X_{\eta}\right\|_{L_{2}\left(\Pi^{(j)}\right)} \\
\left\|\xi_{2}^{m+p+1} \nabla_{\xi} \frac{\partial^{m} X_{\eta}}{\partial \xi_{2}^{m}}\right\|_{L_{2}\left(\Pi^{(j)}\right)} & \leqslant C\left\|X_{\eta}\right\|_{L_{2}\left(\Pi^{(j)}\right)}
\end{aligned}
$$

откуда в силу утверждения 1) и равенства (4.17) вытекает утверждение 2). Лемма доказана.

ЛЕмма 4.3. Функиия $v_{2}^{\text {odd }}$ удовлетворяет оценкам

$$
\begin{gathered}
\left\|\xi_{2}^{p} v_{2}^{\text {odd }}\right\|_{L_{2}\left(\Pi^{(j)}\right)} \leqslant C|\ln \sin \eta|^{1 / 2}, \quad\left\|\xi_{2}^{p} \nabla_{\xi} v_{2}^{\text {odd }}\right\|_{L_{2}\left(\Pi^{(j)}\right)} \leqslant C|\ln \sin \eta|^{1 / 2} \\
\left\|\xi_{2}^{p} \nabla_{\xi} \frac{\partial}{\partial \xi_{2}} v_{2}^{\text {odd }}\right\|_{L_{2}\left(\Pi^{(j)}\right)} \leqslant C|\ln \sin \eta|^{1 / 2},
\end{gathered}
$$

где $p \geqslant 0, p \in \mathbb{Z}$, а константы $C$ не зависят от $\eta$. 
ДокАЗАТЕЛЬСТво. Пусть $v \in \mathscr{V}_{\eta} \cap H^{1}\left(\Pi^{(j)}\right)$ - нечетная по $\xi_{1}$ функция, являющаяся решением краевой задачи

$$
\Delta_{\xi} v=f, \quad \xi_{2}>0, \quad v=0, \quad \xi \in \gamma^{\eta}, \quad \frac{\partial v}{\partial \xi_{2}}=0, \quad \xi \in \Gamma^{\eta}
$$

где $f \in \mathscr{V}_{\eta} \cap L_{2}\left(\Pi^{(j)}\right)$ нечетна по $\xi_{1}$. Поскольку $v \in \mathscr{V}_{\eta}$ нечетна по $\xi_{1}$, то $v=0$ при $\xi_{1}=\pi k / 2, k \in \mathbb{Z}$. Следовательно,

$$
v(\xi)=\int_{-\pi / 2+\pi j}^{\xi_{1}} \frac{\partial v}{\partial t}\left(t, \xi_{2}\right) d t
$$

откуда в силу неравенства Коши-Буняковского вьводим оценку

$$
|v(\xi)|^{2} \leqslant \pi \int_{-\pi / 2+\pi j}^{\pi / 2+\pi j}\left|\frac{\partial v}{\partial \xi_{1}}(\xi)\right|^{2} d \xi_{1}
$$

используя которую, окончательно получаем

$$
\|v\|_{L_{2}\left(\Pi^{(j)}\right)} \leqslant \pi\left\|\nabla_{\xi} v\right\|_{L_{2}\left(\Pi^{(j)}\right)} \cdot
$$

Теперь умножим уравнение из (4.19) на $v$ и один раз проинтегрируем по частям. Получим

$$
\left\|\nabla_{\xi} v\right\|_{L_{2}\left(\Pi^{(j)}\right)}=-\int_{\Pi^{(j)}} v f d \xi
$$

откуда в силу неравенства Коши-Буняковского и оценки (4.20) имеем

$$
\|v\|_{L_{2}\left(\Pi^{(j)}\right)} \leqslant \pi^{2}\|f\|_{L_{2}\left(\Pi^{(j)}\right)}, \quad\|\nabla v\|_{L_{2}\left(\Pi^{(j)}\right)} \leqslant \pi\|f\|_{L_{2}\left(\Pi^{(j)}\right)} .
$$

Применяя неравенства (4.21) к решению задачи (4.12) и учитывая лемму 4.2, получаем равномерные по $\eta$ оценки

$$
\begin{gathered}
\left\|v_{2}^{\text {odd }}\right\|_{L_{2}\left(\Pi^{(j)}\right)} \leqslant C\left\|\nabla_{\xi} X_{\eta}\right\|_{L_{2}\left(\Pi^{(j)}\right)} \leqslant C|\ln \sin \eta|^{1 / 2} \\
\left\|\nabla_{\xi} v_{2}^{\text {odd }}\right\|_{L_{2}\left(\Pi^{(j)}\right)} \leqslant C|\ln \sin \eta|^{1 / 2}
\end{gathered}
$$

Далее, функции $\xi_{2}^{p} v_{2}^{\text {odd }}$ являются решением задачи (4.19), где $\gamma_{\eta}$ совпадает с осью $\xi_{1}$, а правые части имеют вид

$$
f=p(p-1) \xi_{2}^{p-2} v_{2}^{\text {odd }}+2 p \xi_{2}^{p-1} \frac{\partial v_{2}^{\text {odd }}}{\partial \xi_{2}}+\xi_{2}^{p} \frac{\partial X_{\eta}}{\partial \xi_{1}}
$$

Поэтому, применяя к $\xi_{2}^{p} v_{2}^{\text {odd }}$ оценки $(4.21)$, с учетом (4.22) и леммы 4.2 получаем

$$
\begin{aligned}
\left\|\xi_{2} v_{2}^{\text {odd }}\right\|_{L_{2}\left(\Pi^{(j)}\right)} \leqslant & C\left(\left\|\nabla v_{2}^{\text {odd }}\right\|_{L_{2}\left(\Pi^{(j)}\right)}+\left\|\nabla X_{\eta}\right\|_{L_{2}\left(\Pi^{(j)}\right)}\right) \leqslant C|\ln \sin \eta|^{1 / 2} \\
\left\|\xi_{2}^{p} v_{2}^{\text {odd }}\right\|_{L_{2}\left(\Pi^{(j)}\right)} \leqslant & C\left(\left\|\xi_{2}^{p-2} v_{2}^{\text {odd }}\right\|_{L_{2}\left(\Pi^{(j)}\right)}+\left\|\xi_{2}^{p-1} \nabla v_{2}^{\text {odd }}\right\|_{L_{2}\left(\Pi^{(j)}\right)}\right. \\
& \left.+\left\|\xi_{2}^{p} \nabla_{\xi} X_{\eta}\right\|_{L_{2}\left(\Pi^{(j)}\right)}\right), \quad p \geqslant 2 .
\end{aligned}
$$


Интегрируя по частям в равенствах $(m \geqslant 1, m \in \mathbb{Z})$

$$
\begin{aligned}
\int_{\Pi^{(j)}} \xi_{2}^{2 m} v_{2}^{\text {odd }} \frac{\partial X_{\eta}}{\partial \xi_{1}} d \xi & =\int_{\Pi^{(j)}} \xi_{2}^{2 m} v_{2}^{\text {odd }} \Delta_{\xi} v_{2}^{\text {odd }} d \xi \\
\int_{\Pi^{(j)}} \xi_{2}^{2(m+1)} \frac{\partial v_{2}^{\text {odd }}}{\partial \xi_{2}} \frac{\partial^{2} X_{\eta}}{\partial \xi_{1} \partial \xi_{2}} d \xi & =\int_{\Pi^{(j)}} \xi_{2}^{2(m+1)} \frac{\partial v_{2}^{\text {odd }}}{\partial \xi_{2}} \Delta_{\xi} \frac{\partial v_{2}^{\text {odd }}}{\partial \xi_{2}} d \xi
\end{aligned}
$$

аналогично тому, как было получено (4.18), выводим неравенства

$$
\begin{gathered}
\left\|\xi_{2}^{m} \nabla_{\xi} v_{2}^{\text {odd }}\right\|_{L_{2}\left(\Pi^{(j)}\right)} \leqslant C\left(\left\|\xi_{2}^{m-1} v_{2}^{\text {odd }}\right\|_{L_{2}\left(\Pi^{(j)}\right)}+\left\|\xi_{2}^{m+1} \frac{\partial X_{\eta}}{\partial \xi_{1}}\right\|_{L_{2}\left(\Pi^{(j)}\right)}\right) \\
\left\|\xi_{2}^{m+1} \nabla_{\xi} \frac{\partial v_{2}^{\text {odd }}}{\partial \xi_{2}}\right\|_{L_{2}\left(\Pi^{(j)}\right)} \leqslant C\left(\left\|\xi_{2}^{m} \nabla_{\xi} v_{2}^{\text {odd }}\right\|_{L_{2}\left(\Pi^{(j)}\right)}+\left\|\xi_{2}^{m+2} \frac{\partial^{2} X_{\eta}}{\partial \xi_{1} \partial \xi_{2}}\right\|_{L_{2}\left(\Pi^{(j)}\right)}\right) .
\end{gathered}
$$

Из полученных неравенств, леммы 4.2 и оценок (4.22), (4.23) по индукции вытекает утверждение леммы. Лемма доказана.

Лемма 4.4. Для любого $R>0$ и иелого $m \geqslant 3$ имеют место равномерные по $R$ и п оценки $(k=0,1,2)$

$$
\begin{aligned}
&\left\|X_{\eta}\right\|_{L_{2}\left(\Pi^{(j)} \cup\left\{\xi: \xi_{2}>R\right\}\right)} \leqslant C R^{-m}\left(\frac{\pi}{2}-\eta\right)^{2}\left(\left|\ln \left(\frac{\pi}{2}-\eta\right)\right|+1\right), \\
&\left\|\xi_{2}^{k} \nabla_{\xi} X_{\eta}\right\|_{L_{2}\left(\Pi^{(j)} \cup\left\{\xi: \xi_{2}>R\right\}\right)} \leqslant C R^{-m}\left(\frac{\pi}{2}-\eta\right)^{2}\left(\left|\ln \left(\frac{\pi}{2}-\eta\right)\right|+1\right), \\
&\left\|\xi_{2}^{k+1} \nabla_{\xi} \frac{\partial X_{\eta}}{\partial \xi_{2}}\right\|_{L_{2}\left(\Pi^{(j)} \cup\left\{\xi: \xi_{2}>R\right\}\right)} \leqslant C R^{-m}\left(\frac{\pi}{2}-\eta\right)^{2}\left(\left|\ln \left(\frac{\pi}{2}-\eta\right)\right|+1\right), \\
&\left\|v_{2}^{\text {odd }}\right\|_{L_{2}\left(\Pi^{(j)} \cup\left\{\xi: \xi_{2}>R\right\}\right)} \leqslant C R^{-m}|\ln \sin \eta|^{1 / 2}, \\
&\left\|\nabla_{\xi} v_{2}^{\text {odd }}\right\|_{L_{2}\left(\Pi^{(j)} \cup\left\{\xi: \xi_{2}>R\right\}\right)} \leqslant C R^{-m}|\ln \sin \eta|^{1 / 2} .
\end{aligned}
$$

ДокаЗАТЕЛЬСТво. Для любой функции $v \in \mathscr{V}_{\eta}$ при $\xi_{2} \geqslant R$ в силу неравенства Коши-Буняковского справедливы соотношения

$$
|v(\xi)|=\left|\int_{\xi_{2}}^{+\infty} \frac{\partial v}{\partial t}\left(\xi_{1}, t\right) d t\right| \leqslant \frac{1}{\sqrt{2 m-3}} \xi_{2}^{-m+3 / 2}\left\|\xi_{2}^{m-1} \frac{\partial v}{\partial \xi_{2}}\right\|_{L_{2}\left(\mathbb{R}_{+}\right)} .
$$

Интегрируя это неравенство по $\Pi^{(j)} \cap\left\{\xi: \xi_{2}>R\right\}$, получаем

$$
\|v\|_{L_{2}\left(\Pi^{(j)} \cap\left\{\xi: \xi_{2}>R\right\}\right)} \leqslant \frac{R^{-m}}{\sqrt{(2 m-3)(2 m-4)}}\left\|\xi_{2}^{m-1} \frac{\partial v}{\partial \xi_{2}}\right\|_{L_{2}\left(\Pi^{(j)} \cap\left\{\xi: \xi_{2}>R\right\}\right)} .
$$

Последовательно полагая в данном неравенстве

$$
v=X_{\eta}, \quad v=\xi_{2}^{k} \frac{\partial X_{\eta}}{\partial \xi_{i}}, \quad v=\xi_{2}^{k+1} \frac{\partial^{2} X_{\eta}}{\partial \xi_{i} \partial \xi_{2}}, \quad v=v_{2}^{\text {odd }}, \quad v=\frac{\partial v_{2}^{\text {odd }}}{\partial \xi_{i}}, \quad i=1,2,
$$

приходим к утверждению леммы. Лемма доказана. 
Лемма 4.5. Функиии $\lambda_{1}(\varepsilon)$ u $\psi_{1}(x, \varepsilon) \in C^{\infty}(\bar{\Omega})$ ограничены по $\varepsilon$ :

$$
\left|\lambda_{1}\right| \leqslant C, \quad\left\|\psi_{1}\right\|_{C^{3}(\bar{\Omega})} \leqslant C .
$$

ДоКАЗАТЕЛЬСТвО. Ограниченность $\lambda_{1}(\varepsilon)$ следует из условия $(\mathrm{C} 0)$ и формулы (4.10). Гладкость функции $\psi_{1}$ очевидна. В силу хорошо известных оценок решений эллиптических краевых задач для функции $\psi_{1}$ имеем

$$
\left\|\psi_{1}\right\|_{H^{2}(\Omega)} \leqslant C\left(\left|\lambda_{1}\right|\left\|\psi_{0}\right\|_{L_{2}(\Omega)}+\left\|\psi_{0}^{\nu} / \theta_{\varepsilon}^{\prime}\right\|_{C^{2}(\partial \Omega)}\right) \leqslant C,
$$

где $C$ не зависит от $\varepsilon$, откуда в силу теоремы вложения $H^{2}(\Omega)$ в $C(\Omega)$ получаем, что $\left\|\psi_{1}\right\|_{C(\bar{\Omega})} \leqslant C$ с не зависящей от $\varepsilon$ константой $C$. Применяя теперь оценки Шаудера (см. [36, гл. III, $\S 1$, формула (1.11)]) и учитьвая ограниченность нормы $\left\|\theta_{\varepsilon}^{\prime}\right\|_{C^{3+c_{3}(\partial \Omega)}}$, выводим

$$
\left\|\psi_{1}\right\|_{C^{3}(\bar{\Omega})} \leqslant C\left(\left|\lambda_{1}\right|\left\|\psi_{0}\right\|_{C^{2}(\bar{\Omega})}+\left\|\psi_{1}\right\|_{C(\bar{\Omega})}+\left\|\psi_{0}^{\nu} / \theta_{\varepsilon}^{\prime}\right\|_{C^{3+c_{3}(\partial \Omega)}}\right) \leqslant C,
$$

где $C$ не зависит от $\varepsilon$. Лемма доказана.

Пусть

$$
\begin{gathered}
\hat{\lambda}_{\varepsilon}=\lambda_{0}+\varepsilon \lambda_{1} \ln \sin \eta \\
\widehat{\psi}_{\varepsilon}(x)=\psi_{0}(x)+\varepsilon \ln \sin \eta \psi_{1}(x, \varepsilon)+\chi\left(\tau / c_{0}\right) \psi_{\varepsilon}^{\mathrm{bl}}(\xi, s, \eta)+R_{\varepsilon}(x), \\
R_{\varepsilon}(x)=\varepsilon^{2} \frac{\chi\left(\tau / c_{0}\right) \psi_{0}^{\nu}}{\theta_{\varepsilon}^{\prime}} \ln ^{2} \sin \eta
\end{gathered}
$$

где $\lambda_{1}$ определяется формулой (4.10), $\psi_{\varepsilon}^{\mathrm{bl}}$ - формулой (4.3) с $v_{1}$ и $v_{2}$ из (4.7) и (4.11).

Следуюшее утверждение является аналогом леммы 2.6.

ЛЕмма 4.6. Функиия $\widehat{\psi}_{\varepsilon} \in C^{\infty}\left(\Omega \cup \gamma_{\varepsilon} \cup \Gamma_{\varepsilon}\right) \cap H^{1}(\Omega)$ сходится $\kappa \psi_{0}$ в $H^{1}(\Omega)$ и удовлетворяет краевой задаче (2.50) при $u_{\varepsilon}=\widehat{\psi}_{\varepsilon}, \lambda=\lambda_{\varepsilon}, f=f_{\varepsilon}$, где для $f_{\varepsilon}$ верна равномерная оценка

$$
\left\|f_{\varepsilon}\right\|_{L_{2}(\Omega)} \leqslant C \varepsilon^{3 / 2}\left(|\ln \eta|^{3 / 2}+1\right)\left(\frac{\pi}{2}-\eta\right),
$$

константа $C$ не зависит от $\varepsilon$ и $\eta$. Для функции $R_{\varepsilon} \in C^{\infty}(\bar{\Omega})$ верна равномерная по в и $\eta$ оценка

$$
\left\|R_{\varepsilon}\right\|_{C^{2}(\bar{\Omega})} \leqslant C \varepsilon^{2} \ln ^{2} \sin \eta
$$

ДокАЗАТЕльство. Гладкость $\widehat{\psi}_{\varepsilon}$ и $R_{\varepsilon}$ является прямым следствием определения этих функций. Утверждаемые граничные условия для $\widehat{\psi}_{\varepsilon}$ вытекают из (4.5), (4.6), (4.8), (4.9), (4.12). Доказательство оценки для $R_{\varepsilon}$ основывается на лемме 4.5 и условии $(\mathrm{C} 0)$ :

$$
\left\|R_{\varepsilon}\right\|_{C^{2}(\bar{\Omega})} \leqslant C \varepsilon^{2} \ln ^{2} \sin \eta\left\|\psi_{1}\right\|_{C^{3}(\bar{\Omega})} \leqslant C \varepsilon^{2} \ln ^{2} \sin \eta .
$$


Докажем оценку для $f_{\varepsilon}$. Эта функция представляется в виде

$$
\begin{aligned}
f_{\varepsilon} & =-\left(\Delta_{x}+\widehat{\lambda}_{\varepsilon}\right) \widehat{\psi}_{\varepsilon}=-\sum_{i=1}^{3} f_{\varepsilon}^{(i)}, \\
f_{\varepsilon}^{(1)} & =\varepsilon^{2} \ln ^{2} \sin \eta\left(\lambda_{1} \psi_{1}+\left(\Delta+\widehat{\lambda}_{\varepsilon}\right) \chi\left(\tau / c_{0}\right) \psi_{0}^{\nu} / \theta_{\varepsilon}^{\prime}\right), \\
f_{\varepsilon}^{(2)} & =\chi\left(\tau / c_{0}\right)\left(\Delta_{x}+\widehat{\lambda}_{\varepsilon}\right) \psi_{\varepsilon}^{\mathrm{bl}}, \\
f_{\varepsilon}^{(3)} & =2\left(\nabla_{x} \psi_{\varepsilon}^{\mathrm{bl}}, \nabla_{x} \chi\left(\tau / c_{0}\right)\right)+\psi_{\varepsilon}^{\mathrm{bl}} \Delta_{x} \chi\left(\tau / c_{0}\right) .
\end{aligned}
$$

Функция $f_{\varepsilon}^{(1)}$ легко оценивается благодаря лемме 4.5 :

$$
\left\|f_{\varepsilon}^{(1)}\right\|_{L_{2}(\Omega)} \leqslant C \varepsilon^{2} \ln ^{2} \sin \eta
$$

где $C$ не зависит от $\varepsilon$ и $\eta$. Поскольку $\nabla_{x} \chi\left(\tau / c_{0}\right)$ и $\Delta_{x} \chi\left(\tau / c_{0}\right)$ не равны нулю лишь при $c_{0} / 4<\tau<3 c_{0} / 4$, то, учитывая определение переменных $\xi$ и используя лемму 4.4 при $m=3$ и $R=c_{0} c_{1} /(4 \varepsilon)$ (здесь $c_{1}$ - такое, как в условии $(\mathrm{C} 0)$ ), получаем оценку

$$
\left\|f_{\varepsilon}^{(2)}\right\|_{L_{2}(\Omega)} \leqslant C \varepsilon^{7 / 2}|\ln \sin \eta|^{1 / 2}
$$

где $C$ не зависит от $\eta$ и $\varepsilon$. Используя гармоничность $X$ и уравнение для $v_{2}^{\text {odd }}$, приходим к представлению для функции $f_{\varepsilon}^{(3)}$ :

$$
\begin{aligned}
f_{\varepsilon}^{(3)}= & \varepsilon \sum_{k=0}^{2} \sum_{i=1}^{2}\left((\ln \sin \eta)^{\left[\frac{3-k}{2}\right]} G_{4 k+2 i-1}+\xi_{2} G_{4 k+2 i}\right) \xi_{2}^{2\left[\frac{k+1}{2}\right]} \frac{\partial^{k+1} X}{\partial \xi_{i} \partial \xi_{2}^{k}} \\
& +\varepsilon\left(\varepsilon^{2} \ln ^{2} \sin \eta G_{13}+\varepsilon \ln \sin \eta G_{14}+G_{15}\right) X \\
& +\varepsilon \sum_{k=0}^{1} \sum_{i=1}^{2} \xi_{2}^{k} G_{2 k+i+15} \frac{\partial^{k+1} v_{2}^{\text {odd }}}{\partial \xi_{i} \partial \xi_{2}^{k}} \\
& +\varepsilon^{2}\left(\varepsilon \ln \sin \eta G_{20}+G_{21}\right) v_{2}^{\text {odd }}
\end{aligned}
$$

где $G_{i}=G_{i}\left(\xi_{2} ; s, \varepsilon\right)$ - полиномы по $\xi_{2}$ с коэффициентами, зависяшими от $s$ и $\varepsilon$, причем в силу леммы 4.5 эти коэффициенты ограничены равномерно по $s$ и $\varepsilon,[\cdot]-$ целая часть числа. Учитывая эти оценки и применяя леммы 4.2 и 4.3, получаем

$$
\left\|f_{\varepsilon}^{(2)}\right\|_{L_{2}(\Omega)} \leqslant C \varepsilon^{3 / 2}\left(|\ln \eta|^{3 / 2}+1\right)\left(\frac{\pi}{2}-\eta\right)
$$

где $C$ не зависит от $\varepsilon$ и $\eta$. Здесь мы также использовали следующие очевидные соотношения: $\ln \sin \eta=O(\eta), \eta \rightarrow 0 ; \ln \sin \eta=O\left((\pi / 2-\eta)^{2}\right), \eta \rightarrow \pi / 2$. Лемма доказана.

Обоснование построенных асимптотик проводится аналогично обоснованию асимптотик из $\S 2$.

Формальное построение асимптотик в случае кратного предельного собственного значения принципиально не отличается от случая простого предельного собственного значения. Отличием будет лишь то, что одновременно строятся асимптотики всех собственных значений, сходящихся к кратному предельному собственному значению; в целом же формальное построение дословно повторяет приведенные выше рассуждения. Обоснование асимптотик в случае кратного предельного собственного значения также аналогично обоснованию, приведенному в $§ 2$. Лемма 4.1 полностью доказана. 


\section{$\S 5$. Оценки собственных значений возмущенной задачи}

В настоящем параграфе докажем теоремы 1.1-1.3. Доказательства этих теорем будут основываться на следуюшем вспомогательном утверждении.

ЛЕмма 5.1. Пусть множества $\gamma_{1}(\varepsilon), \gamma_{2}(\varepsilon) \subseteq \partial \Omega$ таковы, что $\gamma_{1}(\varepsilon) \subseteq$ $\gamma_{2}(\varepsilon), \quad \lambda_{\varepsilon, 1}^{k}, \lambda_{\varepsilon, 2}^{k}-$ собственные значения возмущенной задачи при $\gamma_{\varepsilon}=\gamma_{1}(\varepsilon)$ $u \gamma_{\varepsilon}=\gamma_{2}(\varepsilon)$ соответственно, взятые с учетом кратности в порядке неубывания. Тогда для всех $k$ верны неравенства

$$
\lambda_{\varepsilon, 1}^{k} \leqslant \lambda_{\varepsilon, 2}^{k}
$$

Лемма 5.1 представляет собой стандартное утверждение о вариационных свойствах собственных значений эллиптических краевых задач, доказательство основано на минимаксном свойстве собственных значений и очевидном вложении функциональных пространств: $H^{1}\left(\Omega, \gamma_{2}(\varepsilon)\right) \subseteq H^{1}\left(\Omega, \gamma_{1}(\varepsilon)\right)$, где $H^{1}\left(\Omega, \gamma_{i}(\varepsilon)\right), i=1,2,-$ множество функций из $H^{1}(\Omega)$, обращающихся в нуль на $\gamma_{i}(\varepsilon)$.

ДокАЗАтЕЛЬСтво теоремы 1.1. Согласно теореме Лагранжа функции $a^{j}$ и $b^{j}$, введенные в $\S 2$, можно выразить через $a_{j}$ и $b_{j}$ следующим образом:

$$
a^{j}=\theta_{\varepsilon}^{\prime}\left(M_{j, \varepsilon}^{(3)}\right) a_{j}, \quad b^{j}=\theta_{\varepsilon}^{\prime}\left(M_{j, \varepsilon}^{(4)}\right) b_{j},
$$

где $M_{j, \varepsilon}^{(3)} \in\left(s_{j}^{\varepsilon}-\varepsilon a_{j}, s_{j}^{\varepsilon}\right), M_{j, \varepsilon}^{(4)} \in\left(s_{j}^{\varepsilon}, s_{j}^{\varepsilon}+\varepsilon b_{j}\right)$. Из полученных представлений в силу условия (C0) и условия 1 ) теоремы вытекает, что

$$
a^{j} \geqslant c_{1} a_{j}, \quad b^{j} \geqslant c_{1} b_{j}
$$

Из этих оценок, условий (С0) и 1$)$ и того факта, что множества $\gamma_{\varepsilon, j}$ не пересекаются, имеем

$$
2 \eta(\varepsilon) \leqslant \min _{j} a^{j}(\varepsilon)+\min _{i} b^{i}(\varepsilon) \leqslant \pi,
$$

т. е. функция $\eta$ ограничена сверху числом $\pi / 2$. Кроме того, из последних неравенств следует существование функций $a_{*}(\varepsilon)$ и $b_{*}(\varepsilon)$ таких, что $a_{*}+b_{*}=2 \eta$, а для множества

$$
\gamma_{\varepsilon, *}=\left\{x: x \in \partial \Omega,-\varepsilon a_{*}(\varepsilon)<\theta_{\varepsilon}(s)-\theta_{\varepsilon}\left(s_{j}^{\varepsilon}\right)<\varepsilon b_{*}(\varepsilon), j=0, \ldots, N-1\right\}
$$

выполнено включение $\gamma_{\varepsilon, *} \subseteq \gamma_{\varepsilon}$. Через $\lambda_{\varepsilon, *}^{k}$ обозначим собственные значения возмушенной задачи при $\gamma_{\varepsilon}=\gamma_{\varepsilon, *}$, взятые с учетом кратности в порядке неубывания. Множество $\gamma_{\varepsilon, *}$ удовлетворяет условиям леммы 4.1 с функцией $\eta$ из условия 1) теоремы. Согласно лемме 4.1 собственные значения $\lambda_{\varepsilon, *}^{k}$ сходятся к собственным значениям $\lambda_{0}^{k}$ задачи (1.4) и для них верны асимптотики из этой леммы. Дважды применяя теперь лемму 5.1 при $\gamma_{1}(\varepsilon)=\gamma_{\varepsilon, *}, \gamma_{2}(\varepsilon)=\gamma_{\varepsilon}$ и $\gamma_{1}(\varepsilon)=\gamma_{\varepsilon}, \gamma_{2}(\varepsilon)=\partial \Omega$, выводим двусторонние оценки

$$
\lambda_{\varepsilon, *}^{k} \leqslant \lambda_{\varepsilon}^{k} \leqslant \lambda_{0}^{k}
$$

Теперь в полученных оценках заменим $\lambda_{\varepsilon, *}^{k}$ на асимптотики из леммы 5.1, что дает, во-первых, сходимость $\lambda_{\varepsilon}^{k}$ к $\lambda_{0}^{k}$ и, во-вторых, требуемые двусторонние оценки разностей $\lambda_{\varepsilon}^{k}-\lambda_{0}^{k}$. Теорема доказана. 
ДОКАЗАТЕЛЬСТВО ТЕОРЕМЫ 1.2. Из первой оценки леммы 2.1 и условия теоремы выводим неравенства

$$
2 \eta_{0} \eta \leqslant a^{j}+b^{j} \leqslant 2 \eta \text {. }
$$

Отсюда вытекает, что, во-первых, функция $\eta_{0}$ ограничена сверху единицей и, во-вторых, найдутся неотрицательные ограниченные функции $a_{*}^{j}(\varepsilon), b_{*}^{j}(\varepsilon), a^{j, *}(\varepsilon)$, $b^{j, *}(\varepsilon)$ такие, что $a_{*}^{j}+b_{*}^{j}=2 \eta_{0} \eta, a^{j, *}+b^{j, *}=2 \eta$, а для множеств

$$
\begin{aligned}
\gamma_{\varepsilon, *} & =\left\{x: x \in \partial \Omega,-\varepsilon a_{*}^{j}(\varepsilon)<\theta_{\varepsilon}(s)-\theta_{\varepsilon}\left(s_{j}^{\varepsilon}\right)<\varepsilon b_{*}^{j}(\varepsilon), j=0, \ldots, N-1\right\}, \\
\gamma_{\varepsilon}^{*} & =\left\{x: x \in \partial \Omega,-\varepsilon a^{j, *}(\varepsilon)<\theta_{\varepsilon}(s)-\theta_{\varepsilon}\left(s_{j}^{\varepsilon}\right)<\varepsilon b^{j, *}(\varepsilon), j=0, \ldots, N-1\right\}
\end{aligned}
$$

выполнены вложения

$$
\gamma_{\varepsilon, *} \subseteq \gamma_{\varepsilon} \subseteq \gamma_{\varepsilon}^{*} .
$$

Через $\lambda_{\varepsilon, *}^{k}$ и $\lambda_{\varepsilon}^{k, *}$ обозначим собственные значения возмушенной задачи при $\gamma_{\varepsilon}=\gamma_{\varepsilon, *}$ и $\gamma_{\varepsilon}=\gamma_{\varepsilon}^{*}$ с учетом кратности в порядке неубывания. Множества $\gamma_{\varepsilon, *}$ и $\gamma_{\varepsilon}^{*}$ удовлетворяют условиям (C0) и (C1): функция $\eta$ из (C1) для них - это функции $\eta_{0} \eta$ и $\eta$ из условия теоремы соответственно; равенство (1.5) для этих функций выполнено с одним и тем же $A>0$. Величины $\delta^{j}(\varepsilon)$ для множеств $\gamma_{\varepsilon, *}$ и $\gamma_{\varepsilon}^{*}$ равны нулю, а потому в силу леммы 2.8 собственные значения $\lambda_{\varepsilon, *}^{k}$ и $\lambda_{\varepsilon}^{k, *}$ сходятся к собственным значениям задачи (1.6) и верны асимптотики

$$
\begin{aligned}
& \lambda_{\varepsilon, *}^{k}=\Lambda_{0}^{k}(\tilde{\mu}, \varepsilon)+\varepsilon(A+\mu)^{2} \int_{\partial \Omega}\left(\Psi_{0}^{k}(x, \mu, \varepsilon)\right)^{2} \theta_{\varepsilon}^{\prime}(s) \ln g_{\varepsilon}\left(\theta_{\varepsilon}(s)\right) d s+O\left(\varepsilon^{3 / 2}\right), \\
& \lambda_{\varepsilon}^{k, *}=\Lambda_{0}^{k}(\mu, \varepsilon)+\varepsilon(A+\mu)^{2} \int_{\partial \Omega}\left(\Psi_{0}^{k}(x, \tilde{\mu}, \varepsilon)\right)^{2} \theta_{\varepsilon}^{\prime}(s) \ln g_{\varepsilon}\left(\theta_{\varepsilon}(s)\right) d s+O\left(\varepsilon^{3 / 2}\right),
\end{aligned}
$$

где

$$
\begin{gathered}
\mu=\mu(\varepsilon)=-(\varepsilon \ln \eta(\varepsilon))^{-1}-A \\
\tilde{\mu}=\tilde{\mu}(\varepsilon)=-\left(\varepsilon \ln \eta_{0}(\varepsilon) \eta(\varepsilon)\right)^{-1}-A=\mu(\varepsilon)+\frac{\left(A^{2}-\mu(\varepsilon)^{2}\right) \varepsilon \ln \eta_{0}(\varepsilon)}{1+(A+\mu(\varepsilon)) \varepsilon \ln \eta_{0}(\varepsilon)} .
\end{gathered}
$$

Из леммы 2.3 выводим оценку $\left\|\Psi_{0}^{k}\right\|_{L_{2}(\partial \Omega)} \leqslant C$ с не зависящей от $\varepsilon$ и $\mu$ константой $C$. Данная оценка, $(2.29)$ и условие $(\mathrm{C} 0)$ позволяют оценить интегралы в (5.2):

$$
-C \leqslant(A+\mu)^{2} \int_{\partial \Omega}\left(\Psi_{0}^{k}\right)^{2} \theta_{\varepsilon}^{\prime} \ln g_{\varepsilon}\left(\theta_{\varepsilon}\right) d s \leqslant 0
$$

где $C>0$ не зависит от $\varepsilon$ и $\mu$. Лемма 5.1 в силу включений (5.1) утверждает справедливость оценок

$$
\lambda_{\varepsilon, *}^{k} \leqslant \lambda_{\varepsilon}^{k} \leqslant \lambda_{\varepsilon}^{k, *},
$$

откуда в силу (5.2), (3.1) и неравенства (5.3) выводим сходимость $\lambda_{\varepsilon}^{k} \rightarrow \lambda_{0}^{k}$ и требуемые двусторонние оценки величин $\lambda_{\varepsilon}^{k}-\lambda_{0}^{k}$. Теорема доказана. 
ДокАЗАТЕЛЬСТво ТЕОРЕмы 1.3. Методика доказательства - такая же, как и в теоремах $1.1,1.2$. Из первой оценки леммы 2.1 и условия теоремы следует, что существуют неотрицательные функции $a^{j, *}(\varepsilon)$ и $b^{j, *}(\varepsilon)$ такие, что $a^{j, *}+b^{j, *}=2 \eta$, и для подмножества границы $\partial \Omega$

$$
\gamma_{\varepsilon}^{*}=\left\{x: x \in \partial \Omega,-\varepsilon a^{j, *}(\varepsilon)<\theta_{\varepsilon}(s)-\varepsilon \pi j<\varepsilon b^{j, *}(\varepsilon), j=0, \ldots, N-1\right\}
$$

выполнено $\gamma_{\varepsilon} \subseteq \gamma_{\varepsilon}^{*}$. Пусть $\lambda_{\varepsilon}^{k, *}-$ собственные значения возмущенной задачи с $\gamma_{\varepsilon}=\gamma_{\varepsilon}^{*}$. Множество $\gamma_{\varepsilon}^{*}$ удовлетворяет всем требованиям теоремы 1.5 с функцией $\eta$ из условия теоремы. Поэтому

$$
\lambda_{\varepsilon}^{k, *}=\lambda_{0}^{k}+\mu \int_{\partial \Omega}\left(\psi_{0}^{k}\right)^{2} \theta_{0}^{\prime} d s+o(\mu)
$$

где $\lambda_{0}^{k}$, напомним, собственные значения задачи (1.6) при $A=0$. Из включений $\varnothing \subseteq \gamma(\varepsilon) \subseteq \gamma^{*}(\varepsilon)$ в силу леммы 5.1 имеем неравенства

$$
\lambda_{0}^{k} \leqslant \lambda_{\varepsilon}^{k} \leqslant \lambda_{\varepsilon}^{k, *}
$$

откуда с учетом асимптотик (5.4) вытекает утверждение теоремы. Теорема доказана.

\section{§6. Приложение}

В настояшем параграфе будут доказаны соотношения (3.1) для собственных значений задачи (1.7), (1.8). Пусть

$$
\widehat{\Lambda}_{0}^{k}=\lambda_{0}^{k}+\mu \lambda_{1}^{k}, \quad \widehat{\Psi}_{0}^{k}=\psi_{0}^{k}+\mu \psi_{1}^{k}+R_{\varepsilon, \mu}^{k}, \quad \lambda_{1}^{k}=\int_{\partial \Omega}\left(\psi_{0}^{k}\right)^{2} \theta_{0}^{\prime} d s .
$$

Функции $\psi_{0}^{k}$, соответствуюшие кратному собственному значению, дополнительно выбраны ортогональными в $L_{2}(\partial \Omega)$ с весом $\theta_{0}^{\prime}$. Функции $\psi_{1}^{k}$ и $R_{\varepsilon, \mu}^{k}$ определяются как решения следуюших задач:

$$
\begin{gathered}
\left(\Delta+\lambda_{0}^{k}\right) \psi_{1}^{k}=-\lambda_{1}^{k} \psi_{0}^{k}, \quad x \in \Omega, \quad\left(\frac{\partial}{\partial \nu}+A \theta_{0}^{\prime}\right) \psi_{1}^{k}=-\theta_{0}^{\prime} \psi_{0}^{k}, \quad x \in \partial \Omega, \\
(\Delta-1) R_{\varepsilon, \mu}^{k}=-\mu^{2} \lambda_{1}^{k} \psi_{1}^{k}, \quad x \in \Omega, \\
\left(\frac{\partial}{\partial \nu}+(A+\mu) \theta_{\varepsilon}^{\prime}\right) R_{\varepsilon, \mu}^{k}=-\left(\theta_{\varepsilon}^{\prime}-\theta_{0}^{\prime}\right)\left((A+\mu) \psi_{0}^{k}+A \mu \psi_{1}^{k}\right)-\mu^{2} \theta_{\varepsilon}^{\prime} \psi_{1}^{k}, \quad x \in \partial \Omega .
\end{gathered}
$$

Задача для $\psi_{1}^{k}$ разрешима, приведенные вьше формула для $\lambda_{1}^{k}$ и условие на $\psi_{0}^{k}$ есть в точности условия разрешимости. Функции $\psi_{1}^{k}$ выберем ортогональными всем собственным функциям, соответствующим $\lambda_{0}^{k}$. Задача для $R_{\varepsilon, \mu}^{k}$, очевидно, однозначно разрешима. Из обших свойств решений эллиптических краевых задач вытекает, что $\psi_{1}^{k}$ и $R_{\varepsilon, \mu}^{k}$ - бесконечно дифференцируемые по $x$ функции, для которых верны оценки

$$
\left\|\psi_{1}^{k}\right\|_{H^{1}(\Omega)} \leqslant C, \quad\left\|R_{\varepsilon, \mu}^{k}\right\|_{H^{1}(\Omega)} \leqslant C\left(\mu^{2}+(A+\mu) \sigma\right)
$$


где константы $C$ не зависят от $\varepsilon$ и $\mu$. Используя эти оценки и определения $\lambda_{1}^{k}$, $\psi_{1}^{k}$ и $R_{\varepsilon, \mu}^{k}$, получаем, что сходящиеся к $\lambda_{0}^{k}$ и $\psi_{0}^{k}$ функции $\widehat{\Lambda}_{0}^{k}$ и $\widehat{\Psi}_{0}^{k}$ удовлетворяют краевой задаче

$$
\begin{gathered}
\left(\Delta+\widehat{\Lambda}_{0}^{k}\right) \widehat{\Psi}_{0}^{k}=\widehat{F}_{k}, \quad x \in \Omega, \quad\left(\frac{\partial}{\partial \nu}+(A+\mu) \theta_{\varepsilon}^{\prime}\right) \widehat{\Psi}_{0}^{k}=0, \quad x \in \partial \Omega, \\
\left\|\widehat{F}_{k}\right\|_{L_{2}(\Omega)} \leqslant C\left(\mu^{2}+(A+\mu) \sigma\right)
\end{gathered}
$$

где константа $C$ не зависит от $\varepsilon$ и $\mu$. Пусть $\lambda_{0}=\lambda_{0}^{q}=\cdots=\lambda_{0}^{q+p-1}-p$-кратное собственное значение. На основе задачи для $\widehat{\Psi}_{0}^{k}$ и оценки правой части $\widehat{F}_{k}$, используя результаты [35], нетрудно доказать, что при $k=q, \ldots, q+p-1$ справедливы следующие представление и равномерная по $\varepsilon$ и $\mu$ оценка:

$$
\widehat{\Psi}_{0}^{k}=\sum_{i=q}^{q+p-1} \frac{\Psi_{0}^{i}}{\Lambda_{0}^{i}-\widehat{\Lambda}_{0}^{k}} \int_{\Omega} \Psi_{0}^{i} \widehat{F}_{k} d x+\hat{u}_{k}, \quad\left\|\hat{u}^{k}\right\|_{H^{1}(\Omega)} \leqslant C\left(\mu^{2}+(A+\mu) \sigma\right) .
$$

Аналогично обоснованию, приведенному в $\S 2$, на основе последних соотношений доказьваются оценки

$$
\left|\Lambda_{0}^{k}-\widehat{\Lambda}_{0}^{k}\right| \leqslant C\left(\mu^{2}+(A+\mu) \sigma\right),
$$

из которых следует справедливость равенств (3.1).

В заключение автор благодарит Р.Р. Гадыльшина за постоянное внимание к работе, обсуждение результатов и полезные замечания.

\section{Список литературы}

1. Damlamian A., Li Ta-Tsien ( Li Daqian). Boundary homogenization for elliptic problems // J. Math. Pures Appl. (9). 1987. V. 66. № 4. P. 351-361.

2. Чечкин $Г$. А. О краевых задачах для эллиптического уравнения второго порядка с осциллирующими граничными условиями // Неклассические дифференциальные уравнения в частных производных. Новосибирск: ИМ СОАН СССР, 1988. С. 95-104.

3. Lobo M., Pèrez M.E. Asymptotic behaviour of an elastic body with a surface having small stuck regions // RAIRO Modélisation Math. Anal. Numér. 1988. V. 22. № 4. P. 609-624.

4. Brillard A., Lobo M., Pèrez M. E. Homogénéisation de frontières par épi-convergence en élasticité linéaire // RAIRO Modélisation Math. Anal. Numér. 1990. V. 24. № 1. P. 5-26.

5. Lobo M., Pèrez M. E. Boundary homogenization of certain elliptic problems for cylindrical bodies // Bull. Sci. Math. 1992. V. 116. Ser. 2. P. 399-426.

6. Чечкин Г. А. Усреднение краевых задач с сингулярным возмущением граничных условий // Матем. сб. 1993. Т. 184. №6. С. 99-150.

7. Беляев А. Г., Чечкин Г. А. Усреднение смешанной краевой задачи для оператора Лапласа в случае, когда "предельная" задача неразрешима // Матем. сб. 1995. Т. 186. № 4. C. $47-60$.

8. Friedman A., Huang Ch., Yong J. Effective permeability of the boundary of a domain // Commun. Partial Differential Equations. 1995. V. 20 (1\&2). P. 59-102.

9. Беляев А.Ю., Чечкин Г.А. Усреднение операторов с мелкомасштабной структурой // Матем. заметки. 1999. Т. 65. № 4. С. 496-510.

10. Barenbaltt G. I., Bell J. B., Crutchfield W. Y. The thermal explosion revisited // Proc. Natl. Acad. Sci. USA. 1998. V. 95. № 23. P. 13384-13386.

11. Dávila J. A nonlinear elliptic equation with rapidly oscillating boundary conditions // Asymptotic Analysis. 2001. V. 28. № 3-4. P. 279-307.

12. Марченко B. А., Хруслов E. Я. Краевые задачи в областях с мелкозернистой границей. Киев: Наук. думка, 1974. 
13. Гадьльшин Р. Р. О модельном аналоге резонатора Гельмгольца в усреднении // Тр. МИАН. 2002. Т. 236. С. 79-86.

14. Олейник O.А., Чечкин Г.А. О краевых задачах для эллиптических уравнений с быстро меняющимся типом граничных условий // УМН. 1993. Т. 48. № 6. С. 163-165.

15. Гадыльшин Р. Р., Чечкин Г. А. Краевая задача для лапласиана с быстро меняющимся типом граничных условий в многомерной области // Сиб. матем. журн. 1999. Т. 40. № 2. C. 271-287.

16. Chechkin G. A., Doronina E. I. On asymptotics of spectrum of boundary value problem with nonperiodic rapidly alternating boundary conditions // Functional Differential Equations. 2001. V. 8. № 1-2. P. 111-122.

17. Гадыльиин Р. Р. Об асимптотике собственных значений для периодически закрепленной мембраны // Алгебра и анализ. 1998. Т. 10. №1. С. 3-19.

18. Гадыльшин P.P. О краевой задаче для лапласиана с быстро осциллируюшими граничными условиями // Докл. РАН. 1998. Т. 362. № 4. С. 456-459.

19. Гадыльшин P. Р. Асимптотики собственных значений краевой задачи с быстро осциллирующими граничными условиями // Диференц. уравнения. 1999. Т. 35. № 4. С. 540-551.

20. Борисов Д. И. О двупараметрической асимптотике в одной краевой задаче для лапласиана // Матем. заметки. 2001. Т. 70. № 4. С. 520-534.

21. Борисов Д.И., Гадьльшин Р.Р. О спектре лапласиана с часто меняющимся типом граничных условий // ТМФ. 1999. Т. 118. № 3. С. 347-353.

22. Гадыльшин P.P. Осреднение и асимптотики в задаче о часто закрепленной мембране // ЖВМиМФ. 2001. Т. 41. № 12. С. 1857-1869.

23. Борисов Д. И. О лапласиане с часто и непериодически чередующимися граничными условиями // Докл. РАН. 2002. Т. 383. № 4. С. 443-445.

24. Filo J., Luckhaus S. Asymptotic expansion for a periodic boundary condition // J. Diff. Equations. 1995. V. 120. № 1. P. 133-173.

25. Filo J. A note on asymptotic expansion for a periodic boundary condition // Arch. Math. (Brno). 1998. V. 34. № 1. P. 83-92.

26. Filo J., Luckhaus $S$. Homogenization of a boundary condition for the heat equation // J. Eur. Math. Soc. 2000. V. 2. № 3. P. 217-258.

27. Borisov D.I. The asymptotics for the eigenelements of the Laplacian in a cylinder with frequently alternating boundary conditions // C. R. Acad. Sci. Paris. Série IIb. 2001. V. 329. № 10. P. 717-721.

28. Борисов Д. И. О сингулярно возмущенной краевой задаче для лапласиана в цилиндре // Дифференц. уравнения. 2002. Т. 38. № 8. С. 1071-1078.

29. Борисов Д. И. О краевой задаче в цилиндре с частой сменой типа граничных условий // Матем. сб. 2002. Т. 193. № 7. С. 37-68.

30. Чечкин Г.А. Полное асимптотическое разложение решения краевой задачи с быстро меняющимися граничными условиями в слое // УМН. 1993. Т. 48. № 4. С. 218-219.

31. Чечкин Г.A. Асимптотическое разложение решения краевой задачи с быстро меняющимся типом граничных условий // Тр. сем. им. И.Г. Петровского. 1996. Т. 19. C. $323-337$.

32. Ильин A. М. Согласование асимптотических разложений решений краевых задач. М.: Наука, 1989.

33. Вишик М.И., Люстерник Л. А. Регулярное вырождение и пограничный слой для линейных дифференциальных уравнений с малым параметром // УМН. 1957. Т. 12. №5. C. $3-122$.

34. Боголюбов Н. Н., Митропольский Ю. А. Асимптотические методы в теории нелинейных колебаний. М.: Наука, 1974.

35. Като T. Теория возмущений линейных операторов. М.: Мир, 1972.

36. Ладыженская O.A., Уральцева H. H. Линейные и квазилинейные уравнения эллиптического типа. М.: Наука, 1973.

37. Планида М. Ю. О сходимости решений сингулярно возмущенных краевых задач для лапласиана // Матем. заметки. 2002. Т. 71. №6. С. 867-877.

E-mail: BorisovDI@ic.bashedu.ru 